\title{
Topological equivalences of the Erdős-Turán Conjecture
}

\author{
Paulo Henrique de Souza Macedo Arruda
}

\author{
DissERTAÇÃO/TESE APRESENTADA \\ $\mathrm{AO}$ \\ Instituto DE MATEMÁticA E EstatísticA \\ DA \\ UniversidADE DE SÃO PAUlo \\ PARA \\ OBTENÇÃO DO TÍTULO \\ DE \\ Mestre em MATEmÁticA \\ Programa: Mestrado em Matemática \\ Orientador: Antônio de Pádua Franco Filho
}

Durante o desenvolvimento deste trabalho o autor recebeu auxílio financeiro do CNPq

São Paulo, Agosto de 2019 


\section{Topological equivalences of the Erdôs-Turán Conjecture}

Esta versão da dissertação contém as correções e alterações sugeridas pela Comissão Julgadora durante a defesa da versão original do trabalho, realizada em 06/09/2019. 


\section{Contents}

Resumo iv

Abstract $\quad$ v

Agradecimentos vi vi vis

Acknowledgements vii

Introduction viii

List of Symbols $\quad 1$

I Order and Topology 3

1 Basic Concepts and Notations 4

2 Lattices and Boolean algebras $\quad 7$

2.1 Order and Choice . . . . . . . . . . . . . . . . . . . . 7

2.2 Lattices . . . . . . . . . . . . . . . . . . . . . . 8

2.3 Filters and Ideals . . . . . . . . . . . . . . . . . . . 10

2.4 Boolean Algebras . . . . . . . . . . . . . . . . . . 12

2.5 Examples . . . . . . . . . . . . . . . . . . . . . 13

3 Topological Spaces and their morphisms 17

3.1 Neighbourhood and bases . . . . . . . . . . . . . . . . 17

3.2 Continuous Functions . . . . . . . . . . . . . . . . . . . . . . . 18 
3.3 Axioms of separation . . . . . . . . . . . . . . . . . 19

3.4 Convergence . . . . . . . . . . . . . . . . . . . . . . . 24

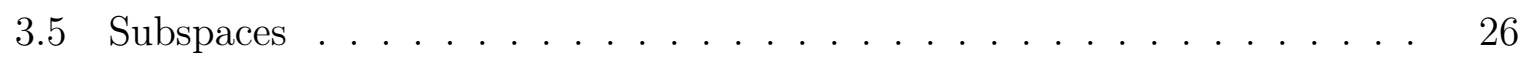

3.6 Product . . . . . . . . . . . . . . . . . . . . 27

3.7 Compactness . . . . . . . . . . . . . . . . . . . . . 29

3.8 The Stone's Duality . . . . . . . . . . . . . . . . . . . . . 30

3.9 Topological translation of order properties . . . . . . . . . . . . . 32

4 Compactifications $\quad 34$

4.1 Extension of continuous functions . . . . . . . . . . . . . . . . 34

4.2 Compactifications . . . . . . . . . . . . . . . . . . 38

4.3 The Čech-Stone Compactification . . . . . . . . . . . . . . . . . . . . 42

II Algebraic Topological Dynamics 45

5 Introductory Concepts $\quad 46$

5.1 Semigroup structure of $\beta \mathbf{N} \ldots \ldots \ldots \ldots$

5.2 Ideals in $\beta \mathbf{N} \ldots \ldots \ldots \ldots \ldots \ldots$. . . . . . . . . . . . . . . . . . . . . . .

5.3 Idempotents in $\beta \mathbf{N} \ldots \ldots \ldots \ldots \ldots$

5.4 Syndetic Sets and the van der Waerden Theorem . . . . . . . . . . . . 53

6 The Erdôs-Turán Conjecture $\quad 59$

6.1 Introduction . . . . . . . . . . . . . . . . . . . . . 59

6.2 The Measure Problem . . . . . . . . . . . . . . . . . . . . 61

6.3 Equivalences of the Erdős-Turán Conjecture . . . . . . . . . . . . . . . . 68

6.4 Final Remarks . . . . . . . . . . . . . . . . . . . . . 76

6.4.1 Szemerédi's Theorem revised . . . . . . . . . . . . . . 77

6.4 .2 Some open questions . . . . . . . . . . . . . . . . . 78

$\begin{array}{lr}\text { Bibliography } & 81\end{array}$

$\begin{array}{lr}\text { Index } & 85\end{array}$ 
The life of Aristotle may be resumed in one single sentence: Aristotle was born, he thought and he died. Everything else that could be said on the matter is pure anedocte.

Martin Heidegger; as cited by J. Derrida in K. Dick and A. Z. Kofman (2002) Derrida.

Asking whether and how a proposition can be verified is only a particular way of asking "How d'you mean?". The answer is a contribution to the grammar of the proposition.

Ludwig Wittgenstein; in [Wit58]

Human beings do not live in the objective world alone, nor alone in the world of social activity as ordinarily understood, but are very much at the mercy of the particular language which has become the medium of expression for their society. It is quite an illusion to imagine that one adjusts to reality essentially without the use of language and that language is merely an incidental means of solving specific problems of communication or reflection. The fact of the matter is that the "real world" is to a large extent unconsciously built upon the language habits of the group.

Edward Sapir; in [Sap29]

Zeno was concerned, as a matter of fact, with three problems, each presented by motion, but each more abstract than motion, and capable of a purely arithmetical treatment. These are the problems of the infinitesimal, the infinite, and continuity. To state clearly the difficulties involved, was to accomplish perhaps the hardest part of the philosopher's task. This was done by Zeno. From him to our own day, the finest intellects of each generation in turn attacked the problems, but achieved, broadly speaking, nothing. In our own time, however, three men Weierstrass, Dedekind, and Cantor - have not merely advanced the three problems, but have completely solved them.

Russell, Bertrand; in Mathematics and the metaphysician, [Rus08]. 


\section{Resumo}

O objetivo desse trabalho foi estudar caminhos topológicos para a teoria dos números, em especial a conjectura de Erdős-Turán acerca da soma dos recíprocos que, no momento no qual este trabalho se conclui, encontra-se aberta. As ferramentas usadas foram a estrutura de semigrupo e a dinâmica que essa estrutura imprime à compactificação de Čech-Stone dos números naturais por translação. Para tal objetivo, foram abordados temas caros à teoria de Ramsey, combinatória, teoria ergódica e teoria combinatória dos números, além da construção dos objetos topológicos e bem como suas estruturas algébricas.

O principal resultado, atribuído a N. Hindman, é uma série de equivalências topológicas da conjectura supracitada. Outros resultados conhecidos, tais como os Teoremas de van der Waerden e Szemerédi são abordados com o mesmo olhar topológico. 


\section{Abstract}

The main goal of this project was study a topological path for number theory, specially the Erdôs-Turán Conjecture on the sum of reciprocals which, at the present moment when this work is being concluded, lies without an answer. The machinery used was the semigroup structure and the dynamics of translations of the Cech-Stone compactification of the natural numbers. For this end, here follows themes dear to Ramsey theory, combinatorics, ergodic theory and combinatorial number theory, likewise all topological and algebraic structures underlying the construction and manipulation of all objects hereon.

The major result described in this document, due to N. Hindman, was equivalences of the aforementioned conjecture written within a topological framework. Other important results also discussed thereon with this framework was the Theorems of van der Waerden and Szemerédi. 


\section{Agradecimentos}

Em primeiro lugar, gostaria de agradecer a todos os meus professores, desde o primário até a pós-graduação; sem eles, o presente trabalho não seria possível. Entre esses, um agradecimento especial ao Prof. Dr. Antônio de Pádua Franco Filho, meu orientador, pelo suporte e confiança durante o mestrado, e a Ana Paula Sanches por ter possibilitado a conclusão desse trabalho.

Esse trabalho é dedicado a todos meus amigos, em especial Bruno Hideki Fukushima Kimura, Alessandro Marins, Thiago Raszeja, Yuri Morais Gustavo, Michel Gaspar, Leonardo Leite, Flávio Cação, João Lucas Micheleto, João Lucas Martini, Luis Gall, Diego Gamma e Gabriela Pelozoni Lima. 


\section{Acknowledgements}

Firstly, I would like to thank all my teachers and professors, from elementary school to graduate school; without them the present text would not be possible; among them, a special thanks to Prof. Dr. Antônio de Pádua Franco Filho, my advisor, for trusting and supporting me during my graduate studies, and to Ana Paula Sanches who made the conclusion of this work possible.

My work here is dedicated to all my friends, specially Bruno Hideki Fukushima Kimura, Alessandro Marins, Thiago Raszeja, Yuri Morais Gustavo, Michel Gaspar, Leonardo Leite, Flávio Cação, João Lucas Micheleto, João Lucas Martini, Luis Gall, Diego Gamma and Gabriela Pelozoni Lima. 


\section{Introduction}

There are several distinct definitions of a large set in mathematics; for example, one can look at the cardinality of two sets to express the fact that one is larger than the other. Although $\mathbf{N}$ and $\left\{2^{n}: n \in \mathbf{N}\right\}$ have the same cardinality, the series

$$
\sum_{n \geq 1} \frac{1}{n} \text { and } \quad \sum_{n \geq 0} \frac{1}{2^{n}}
$$

have different convergence natures. Thus, one can state that $\mathbf{N}$ is larger than $\left\{2^{n}: n \in \mathbf{N}\right\}$ in this sense. Following the same reasoning, the set of all prime numbers is a large set, as proved by Euler in 1737. Also, any infinite arithmetical sequence is a large set. If $A$ is a large set, then, for any natural number $n, A+n$ and $A \cdot n$ are large sets.

In 1927, van der Waerden proved that any colouring of the natural numbers is composed by at least one set containing arbitrarily long arithmetical progressions. This leads the mathematical community to suspect that the aforementioned notion of largeness has a close relationship with the containment of arbitrarily long arithmetical progressions.

The present work will investigate some results of combinatorial number theory under a topological framework. For this end, the theory of compactification and the theory of ultrafilters will be crucial. Ultrafilters that has some sets in the form $A \cup B$, has to contain either $A$ or $B$. Also, the set of all ultrafilters of $\wp(\mathbf{N})$ is the compactification of Cech-Stone of the natural numbers; over this set is possible to extend the usual notions of addition and multiplication of natural numbers that allow the transfer of some number theoretical properties to topologic properties. 


\section{List of Symbols}

\begin{tabular}{|c|c|}
\hline $\mathscr{K}(X)$ & $\begin{array}{l}\text { A transversal set for all compactification of a Ty- } \\
\text { chonoff space } X \text {. }\end{array}$ \\
\hline $\operatorname{AP}_{k}(a, b)$ & $\begin{array}{l}\text { The arithmetic progression of length } k \text {, first element } \\
a \text { and rate } b \text {. }\end{array}$ \\
\hline $\mathscr{A} \mathscr{P}$ & $\begin{array}{l}\text { The set of all ultrafilters of } \wp(\mathbf{N}) \text { whose members con- } \\
\text { tain arbitrarily long arithmetic progressions. }\end{array}$ \\
\hline $\mathscr{B}$ & $\begin{array}{l}\text { The set of all ultrafilters of } \wp(\mathbf{N}) \text { whose members have } \\
\text { positive Banach upper density. }\end{array}$ \\
\hline$\bigcap x$ & Intersection of a non empty set $x$ \\
\hline$\bigcup x$ & Union of $x$ \\
\hline $\mathbf{D}(\kappa)$ & The discrete space of cardinality $\kappa$ \\
\hline $\mathrm{cl}_{X}(A)$ & Clousure of $A$ as a subset of the topological space $X$ \\
\hline$\omega$ & The first infinite cardinal and the cardinality of $\mathbf{N}$. \\
\hline $\mathfrak{c}$ & The cardinality of the real line. \\
\hline $\mathscr{D}$ & $\begin{array}{l}\text { The set of all ultrafilters of } \wp(\mathbf{N}) \text { whose members have } \\
\text { positive natural upper density. }\end{array}$ \\
\hline$\wedge$ & Logical conjuction \\
\hline$\rightarrow$ & Implication \\
\hline$[A]^{\kappa}$ & The set of all subsets of $A$ having cardinality $\kappa$ \\
\hline$\exists$ & Existencial quantifier \\
\hline $\mathrm{FC}(X)$ & $\begin{array}{l}\text { The collection of all functionally closed subsets of a } \\
\text { topological space } X\end{array}$ \\
\hline $\mathrm{FO}(X)$ & $\begin{array}{l}\text { The collection of all functionally open subsets of a } \\
\text { topological space } X\end{array}$ \\
\hline$\forall$ & Universal quantifier \\
\hline $\mathbb{P}(A)$ & The filter generated by the set $A$. \\
\hline$\square(A)$ & The ideal generated by the set $A$. \\
\hline$\iota_{X}$ & The identity map of $X$ over himself. \\
\hline$x \in y$ & Membership relation: $x$ is an element of $y$ \\
\hline$\iota_{A \rightarrow X}$ & The inclusion map of $A$ into $X$ \\
\hline $\inf _{\preceq} B$ & $\begin{array}{l}\text { The supremum of } B \text { with respect to the partial order } \\
\preceq \text {. }\end{array}$ \\
\hline$A \cap B$ & Intersection of the sets $A$ and $B$ \\
\hline
\end{tabular}




\begin{tabular}{|c|c|}
\hline $\operatorname{int}_{X}(A)$ & Interior of $A$ as a subset of the topological space $X$ \\
\hline$a \curlyvee b$ & The supremum of $\{a, b\}$ \\
\hline$[A] \leq \kappa$ & $\begin{array}{l}\text { The set of all subsets of } A \text { having cardinality less than } \\
\text { or equal to } \kappa\end{array}$ \\
\hline$[A]^{<\kappa}$ & $\begin{array}{l}\text { The set of all subsets of } A \text { having cardinality less than } \\
\kappa\end{array}$ \\
\hline$a \curlywedge b$ & The infimum of $\{a, b\}$ \\
\hline$K(S)$ & The minimal ideal of a semigroup $S$. \\
\hline $\mathbf{N}$ & The set of all natural numbers. \\
\hline$V_{X}(x)$ & $\begin{array}{l}\text { The collection of all neighbourhoods of a point } x \text { be- } \\
\text { longing to the topological space } X\end{array}$ \\
\hline V & Logical disjunction \\
\hline$\langle x, y\rangle$ & Ordered pair of elements $x$ and $y$ \\
\hline$p-\lim _{s \in S} x_{n}$ & $\begin{array}{l}\text { The set of all } \mathfrak{p} \text {-limits of the collection of points }\left\langle x_{n}\right\rangle \\
\text { within the topological space } X\end{array}$ \\
\hline$\preceq$ & A partial order. \\
\hline$\wp(A)$ & Power set of $A$ \\
\hline $\mathbf{P}$ & The set of all prime numbers. \\
\hline $\mathrm{p}_{s}$ & The projection of $\prod_{s \in S} X_{s}$ on the coordinate $s$. \\
\hline Q & The set of all rational numbers. \\
\hline $\mathbf{R}$ & The set of all real numbers. \\
\hline $\mathrm{C}(X)$ & $\begin{array}{l}\text { The set of all continuous functions defined in } X \text { and } \\
\text { with image in } \mathbf{R} \text {. }\end{array}$ \\
\hline$\leftrightarrow$ & Equivalence \\
\hline $\operatorname{stn} L$ & The collection of all ultrafilters of a lattice $L$ \\
\hline$A \subseteq B$ & $A$ is a subset of $B$ \\
\hline$A+B$ & The set $\{a+b: a \in A \wedge b \in B\}$. \\
\hline$A+s$ & The set $\{a+s: a \in A\}$ \\
\hline $\sup _{\preceq} B$ & $\begin{array}{l}\text { The supremum of } B \text { with respect to the partial order } \\
\preceq \text {. }\end{array}$ \\
\hline$S^{\uparrow}$ & $\bar{T}$ The set of all increasing sequences of natural numbers. \\
\hline $\mathbb{1}$ & The maximum of a bounded lattice. \\
\hline$A \cup B$ & Union of the sets $A$ and $B$ \\
\hline I & The unit interval $[0,1]$ \\
\hline$X^{\kappa}$ & $\begin{array}{l}\text { The product } \prod_{s \in S} X_{s} \text { where } S \text { is any set having car- } \\
\text { dinality } \kappa \text { and, for all } s \in S, X_{s}=X \text {. }\end{array}$ \\
\hline $\mathrm{Z}$ & The set of all integers. \\
\hline 0 & The minimum of a bounded lattice. \\
\hline
\end{tabular}


Part I

Order and Topology 


\section{CHAPTER 1}

\section{Basic Concepts and Notations}

The present work uses concept of set theory and, although this chapter provides a quick recapitulation of these notions, assumes that the reader is already familiar with this field; a good introduction to the subjects can be found in [Kun11; Lev02; Sho67]. For the sake of completeness, this chapter provides a quick - but distant from the ideal - summary of the set-theoretic notions that will be used throughout the text, which also will serve as the methodology of argumentation.

The symbols $\forall$ and $\exists$ denotes the universal and the existential quantifiers, respectively. So that, if $\mathscr{P}$ is a predicate of the set theory, $\forall x \mathscr{P}(x)$ will indicate that every set whose existence can be derived from the axioms of set theory - here the ZFC is assumed — and satisfies the property $\mathscr{P}$; the expression $\exists x \mathscr{P}(x)$ is the statement that grantees the existence of at least one set within ZFC that satisfies the property $\mathscr{P}$.

The symbol $\rightarrow$ denotes the implication, i.e. if $\varphi$ and $\psi$ are two statements of set theory, then $\varphi \rightarrow \psi$ indicate that the statement $\psi$ can be derived from $\varphi$ altogether with the axioms of set theory and the logical rules of inference. The symbol $\leftrightarrow$ denotes the equivalence between two statements $\varphi$ and $\psi$, i.e $\varphi \rightarrow \psi$ and $\psi \rightarrow \varphi$.

The symbols $\wedge$ and $\vee$ will stand for the connectives of Conjuction and disjunction, respectively; i.e. $\varphi \wedge \psi$ is true if and only if both statements are true, while $\varphi \vee \psi$ is true if and only if at least one of the two statements is true.

The symbol $x \in y$ is the de facto unique symbol of the ZFC's language; as a binary predicate it indicates the belonging of an element to a set. Given any $A$ and $B$, the expression $A \subseteq B$ will indicate that every element of $A$ is an element of $B$.

For any set $x$, one can find its union $\bigcup x$ defined as the collection of all objects that are members of some set that belongs to $x$. Particularly, the union of two sets $A$ and $B$ - which is actually the union of the set $\{A, B\}$ - will be denoted as $A \cup B$. If the union is well defined, one can also define the intersection of a non-empty set $x$, denoted by $\bigcap x$, 
as the collection of all objects that simultaneously are members of all sets belonging to $x$. As it goes for the union, the intersection of two sets $A$ and $B$ - which is actually the intersection of the set $\{A, B\}$ - will be denoted by $A \cap B$.

Given any $x$ and $y$, one can define the ordered pair $\langle x, y\rangle$ by the extension of the equivalence $\langle x, y\rangle=\langle u, v\rangle$ if and only if $x=u$ and $y=v$. A binary relation is any set composed by ordered pairs; the membership relation on a binary relation $R$ will be denoted by $x R y$ instead of $\langle x, y\rangle \in R$. Given any relation $R$, one can define the sets

$$
\operatorname{dom} R=\{x \in A: \exists y \in A(x R y)\} \quad \text { and } \quad \operatorname{ran} R=\{y \in A: \exists x \in A(x R y)\} .
$$

named domain and range of $R$, respectivelly. Being $R$ a binary relation, any set containing the range of $R$ is called a codomain of $R$. The domain and codomain of a binary relation $R$ is obtained from the symbols $R: A \rightarrow B$.

A special sort of binary relation is functions. A function $f: A \rightarrow B$ is a binary relation such that each $x \in A$ has only one image $y \in B$. The fact that $x f y$ will be denoted by $f(x)=y$.

A surjection (or surjective function) is any function whose codomain is its range; a injection (or injective function) is any function $f$ such that, for any $y \in \operatorname{ran} f$, one can find only one $x \in \operatorname{dom} f$ such that $f(x)=y$. A function that is both surjective and injective is called a bijection (or bionivocal function).

If there is a bijection $f$ between the sets $A$ and $B$, one can represents the members of $B$ as an image by a function of the members of $A$ as follows: for any $y \in B$ there is only one $x \in A$ satisfying $f(x)=y$; the element $y$ will be represented as $y_{x}$ instead $f(x)$. Thus, $B$ can be written as $\left\langle b_{a}\right\rangle_{a \in A}$ (or merely as $\left\langle b_{a}\right\rangle$ when the context explains the information omitted). This representation is called indexation of $B$ in terms of the elements of $A$.

If $\mathcal{B}$ is a family of sets and $\left\langle B_{a}\right\rangle_{a \in A}$ is a indextation of $\mathcal{B}$, then $\bigcup \mathcal{B}$ and, if $\mathscr{B}$ is non empty, $\cap \mathscr{B}$ will be written as $\bigcup_{a \in A} B_{a}$ and $\bigcap_{a \in A} B_{a}$, respectivelly.

For any set $A, \wp(A)$ will denote the power set of $A$ - formed by all subsets of $A$-, and $|A|$ will denote the cardinality of $A$, an object that is characterize in the following fashion: for any set $B,|A|=|B|$ if there is a bijection $f: A \rightarrow B$. A relation $|A| \leq|B|$ is defined by the existence of an injective function $f: A \rightarrow B$. In the presence of the Axiom of Choice, $|A| \leq|B|$ if and only if there is a surjection $g: B \rightarrow A$

1.0.1 Theorem. (Cantor-Bernstein-Schröder Theorem) If $|A| \leq|B|$ and $|B| \leq \mid A$, then $|A|=|B|$.

Given two sets $A$ and $B$, the ${ }^{A} B$ denotes the collection of all functions $f: A \rightarrow B$. If $f: X \rightarrow Y$ is a function and $A$ is a subset of $X$, then $f \uparrow_{A}$ is the restriction of $f$ to $A$, namely the function $f \uparrow_{A}: A \rightarrow Y$ given by $f \uparrow_{A}(a)=f(a)$.

If $\left\langle X_{s}\right\rangle_{s \in S}$ is a collection of sets, then

$$
\prod_{s \in S} X_{s}=\left\{f \in \bigcup_{s \in S} X_{s}: \forall s \in S\left(f(s) \in X_{s}\right)\right\} .
$$


is the product of the collection $\left\langle X_{s}\right\rangle_{s \in S}$. In the case of $S$ being finite, say $S=\{0,1,2, \ldots, n\}$, then $\prod_{s \in S} X_{s}$ is the collection of all $n$-ordered lists $\left\langle x_{0}, \ldots, x_{n}\right\rangle$ such that, for any $i \in S$, $x_{i} \in X_{i}$.

Cardinal numbers code the cardinality of sets with their arithmetics. Analogously as it happens with natural numbers, cardinal numbers have a induction and recursion principles, so that any set of cardinal numbers admits a minimum element.

For any cardinal $\kappa$ and any set $A$,

$$
[A]^{<\kappa}=\{B \subseteq A:|B|<\kappa\},[A]^{\kappa}=\{B \subseteq A:|B|=\kappa\}
$$

and

$$
[A]^{\leq \kappa}=\{B \subseteq A:|B| \leq \kappa\}
$$

If $\kappa$ is a cardinal number and $X$ is any set, then $X^{\kappa}$ will denote the product $\prod_{s \in S} X_{s}$ where $S$ is any set having cardinality $\kappa$ and, for all $s \in S, X_{s}=X$.

The set of all natural numbers is denoted by $\mathbf{N}$, whose cardinality is $\omega$; inside $\mathbf{N}$, the set of all prime numbers is denoted by $\mathbf{P}$. The set of all integers is $\mathbf{Z}$ and the set of all rational numbers is denoted by $\mathbf{Q}$. Because of the Cantor-Bernstein-Schröder Theorem 1.0.1,

$$
\omega=|\mathbf{N}|=|\mathbf{Z}|=|\mathbf{Q}| .
$$

The real line is denoted by $\mathbf{R}$ and the unit interval $[0,1]$ will be denoted by $\mathbf{I}$. The cardinal $\mathfrak{c}$ will denote the cardinality of $\mathbf{R}$. It is folkloric that

$$
|\wp(\mathbf{N})|=|\mathbf{R}|=\mathfrak{c}=2^{\omega} .
$$

The term progression will be used to name any finite collection of elements within any set, that will often be indexed by its cardinality. Among all progressions, a special attention will be given to arithmetical progression: given natural numbers $a$ and $b$, such that $b>0$, and a natural number $k$, the arithmetical progression of length $k$, first term $a$ and rate $b$ is the progression

$$
a, a+b, a+2 b, \ldots, a+(k-1) b .
$$

Since Cantor and the structuralism philosophy, mathematically started by the Bourbakian School, is quite useful to study sets, structures that furnish these sets and, since the power of a particular set is also a set, structures furnishing sets of sets. Thus, an arithmetical progression of length $k$, first element $a$ and rate $b$ will be the following set

$$
\operatorname{AP}_{k}(a, b)=\{a+t \cdot b: t<k\} .
$$




\section{CHAPTER 2}

\section{Lattices and Boolean algebras}

\subsection{Order and Choice}

A partial order is a pair $\langle A, \preceq\rangle$ in which $\preceq$ is a relation on $A$ satisfying

1. (Reflexivity) for any $x \in A, x \preceq x$;

2. (Atisymmetry) for every elements $x$ and $y$ of $A$ such that $x \preceq y$ and $y \preceq x$, $x=y$;

3. (Transitivity) if $x, y$ and $z$ are elements of $A$ such that $x \preceq y$ and $y \preceq z$, then $x \preceq z$.

If $\langle A, \preceq\rangle$ is a partial order, an element $a \in A$ is said to be $\preceq$-minimal if no element of $A$ other than $a$ is $\preceq$-smaller than $a$. Analogously, one define the notion of $\preceq$-maximal.

Given a partial order $\langle A, \preceq\rangle$ and a non empty subset $B$ of $A$, a element $a \in A$ is said to be $\preceq$-minorant for $B$ if, for any $b \in B, a \preceq b$. Analogously, $a \in A$ is a $\preceq$-majorant for $B$ if no element of $B$ is $\preceq$-greater than $a$. If $a \in B$ is a minorant of $B$, then it is called a $\preceq$-minimum of $B$; analogously, if $a \in B$ is a majorant of $B$, then $a$ is said to be a $\preceq$-maximum of $B$. Obviously, if $B$ admits a minimum (or maximum) element, then this element must be unique, which enable it to be denoted by $\min _{\preceq} B\left(\max _{\preceq} B\right.$ )or simply $\min B(\max B)$.

A subset $B$ of a partially ordered set $\langle A, \preceq\rangle$ admits a $\preceq$-supremum if the set of all $\preceq$-majorant for $B$ has a smallest element, which, in this case — as it is unique will be called $\preceq$-supremum of $B$, or simply supremum of $B$ when it cannot lead to a misunderstanding, and is denoted by $\sup _{\preceq} B$ or simply $\sup B$. Similarly, one defines the notion of $\preceq$-infimum of $B$ which will be denoted by $\inf _{\preceq} B$ or inf $B$. 
Henceforth, a partial order $\langle A, \preceq\rangle$ will be denoted only by its base set $A$, wherein the the relation $\preceq$ will be referenced if needs be. When confusion may appear, the relation will be denoted $\preceq_{A}$ to indicate its action on the set $A$.

Given two elements $a$ and $b$ from a partially ordered set $A$, the supremum of $\{a, b\}$, when it exists, will be denoted by $a \curlyvee b$ and its infimum, when it exists, will be denoted by $a \curlywedge b$.

A partial order $A$ is said to be a total order — or a totally ordered set — if every pair of elements within $A$ are comparable; i.e. for any elements $a$ and $b$ of $A, a \preceq b$ or $b \preceq a$.

2.1.1 Theorem. (Kuratowski-Zorn's Lemma) If every totally ordered subset of a partially ordered set $A$ has a $\preceq$-majorant, then $A$ contains a $\preceq$-maximal element.

One of the axioms of ZFC is the Axiom of Choice; whenever $\mathcal{A}$ is a non empty collection of non empty sets, this axiom garantees the existence of a transversal set of $\mathcal{A}$, i.e. a set $T$ such that, for any $A \in \mathcal{A},|A \cap T|=1$. The Kuratowski-Zorn's Lemma is actually equivalent to the Axiom of Choice, as it can be seen in [Kun11].

\subsection{Lattices}

A partial order $L$ is said to be a lattice if every unordered pair $\{x, y\}$ of elements within $R$ admits a supremum and infimum with respect of the order $\preceq$.

A Lattice $L$ is said to be bounded if it is bounded as a partial order, i.e. there is a minimum and a maximum of $L$, that will be denoted by $\mathbb{O}$ and $\mathbb{1}$, respectively (or, in the case where it is necessary, $\mathbb{O}_{L}$ and $\mathbb{1}_{L}$, respectively).

2.2.1 Lemma. Selecting three elements $x, y$ and $z$ from a lattice $L$, one can prove that:

APL1 $x \curlyvee x=x$ and $x \curlywedge x=x$

APL2 $x \curlyvee y=y \curlyvee x$ and $x \curlywedge y=y \curlywedge x$;

APL3 $x \curlyvee(y \curlyvee z)=(x \curlyvee y) \curlyvee z$ and $x \curlywedge(y \curlywedge z)=(x \curlywedge y) \curlywedge z$; and

$\operatorname{APL4}(x \curlyvee y) \curlywedge y=y$ and $(x \curlywedge y) \curlyvee y=y$.

Moreover, if $L$ is bounded, then

APL5 $x \curlyvee \mathbb{O}=x$ and $x \curlywedge \mathbb{1}=x$.

2.2.2 Lemma. Given two elements $x$ and $y$ of a lattice $L$, the following are equivalent:

OL1 $x \preceq y$;

OL2 $x \curlywedge y=x$ 
OL3 $x \curlyvee y=y$.

The results 2.2 .1 (pag.8) and 2.2.2 (pag.8) characterize (bounded) lattices in an algebraic fashion. The operations $\curlyvee$ and $\curlywedge$ are reflexive APL1 (pag.8), commutative APL2 (pag.8), transitive APL3 (pag.8) and absorbent APL4 (pag.8); in the bounded case, the element $\mathbb{O}$ is the neutral element for $\curlyvee$ and $\mathbb{1}$ is the neutral element for $\curlyvee$. In fact, one can prove the following:

2.2.3 Lemma. Let $L$ be a set yielding two operations $\cdot$ and + fulfilling the instances of APL1 (pag.8)-APL4 (pag.8) whereas - replaces $\curlywedge$ and + replaces $\curlyvee$. Then there exist an order structure within $L$, engendered by the equivalent assertions of 2.2 .2 (pag.8), which allows $L$ to be a lattice by setting, for every elements $x$ and $y$ of $L, x \curlywedge y=x \cdot y$ and $x \curlyvee y=x+y$. If, by chance, there is $\mathbb{O}$ and $\mathbb{1}$ listed among the elements of $L$ satisfying APL5 (pag.8), then $L$ is also bounded.

The operations of supremum and infimum of a lattice also conserve the order as it can be seen bellow:

2.2.4 Lemma. Let $L$ be a lattice and $x, y$ and $z$ be arbitrary elements of $L$ such that $x \preceq y$. Then $x \curlywedge z \preceq y \curlywedge z$ and $x \curlyvee z \preceq y \curlyvee z$.

2.2.5 Lemma. For any lattice $L$ and any of its elements $x, y$ and $z$, one can prove the following:

(2.2.5.1). $(x \curlywedge y) \curlyvee(x \curlywedge z) \preceq x \curlywedge(y \curlyvee z)$;

(2.2.5.2). $x \curlyvee(y \curlywedge z) \preceq(x \curlyvee y) \curlywedge(x \curlyvee z)$.

Proof: In fact,

$$
x \curlywedge y \leq x, x \curlywedge y \preceq y, x \curlywedge z \preceq x \text { and } x \curlywedge z \preceq z .
$$

Then

$$
(x \curlywedge y) \curlyvee(x \curlywedge z) \preceq x \text { and }(x \curlywedge y) \curlyvee(x \curlywedge z) \preceq(y \curlyvee z)
$$

which result in

$$
(x \curlywedge y) \curlyvee(x \curlywedge z) \preceq x \curlywedge(y \curlyvee z) .
$$

The proof of the second inequality is similar.

2.2.6 Lemma. For any lattice $L$ and any elements $x, y$ and $z$ of $L$, the following are equivalent:

$\operatorname{DL1} x \curlywedge(y \curlyvee z) \preceq(x \curlywedge y) \curlyvee(x \curlywedge z) ;$

$\operatorname{DL2}(x \curlyvee y) \curlywedge(x \curlyvee z) \preceq x \curlyvee(y \curlywedge z)$. 
Proof: Assuming DL1 (pag.9), let $w=(x \curlyvee y)$. Then, by APL4 (pag.8), $x \curlywedge w=x$. Thus, using DL1 (pag.9),

$$
(x \curlyvee y) \curlywedge(x \curlyvee z)=w \curlywedge(x \curlyvee z) \preceq(w \curlywedge x) \curlyvee(w \curlywedge z)=x \curlyvee[z \curlywedge(x \curlyvee y)] .
$$

As the hypothesis goes, one has

$$
z \curlywedge(x \curlyvee y) \preceq(z \curlywedge x) \curlyvee(z \curlywedge y)
$$

With the help of the associativity of $\curlyvee$ and APL4 (pag.8), one proves that

$$
x \curlyvee[z \curlywedge(x \curlyvee y)] \preceq x \curlyvee[(z \curlywedge x) \curlyvee(z \curlywedge y)]=[x \curlyvee(z \curlywedge x)] \curlyvee(z \curlywedge y)=x \curlyvee(z \curlywedge y),
$$

which establishes DL2 (pag.9). The implication DL2 (pag.9) $\Longrightarrow$ DL1 (pag.9) is proved by a similar argument.

A lattice that meets the equivalent assertions of 2.2.6 (pag.9) is called distributive lattice. Evidently, using the lemma 2.2 .5 (pag.9), a lattice $L$ is a distributive lattice $L$ if and only if, for any elements $x, y$ and $z$ of $L$, one can prove that

$$
\begin{aligned}
& \text { DL1' } x \curlywedge(y \curlyvee z)=(x \curlywedge y) \curlyvee(x \curlywedge z) ; \\
& \text { DL2' } x \curlyvee(y \curlywedge z)=(x \curlyvee y) \curlywedge(x \curlyvee z) .
\end{aligned}
$$

\section{$2.3 \quad$ Filters and Ideals}

A filter of a lattice $L$ is a collection $P$ of elements within $L$ that satisfies the following properties:

F1 for any two elements $x$ and $y$ of $P, x \curlywedge y \in P$;

F2 if $x \in P$ and $y \in L$ are such that $x \preceq y$, then $y \in P$.

A filter of a lattice $L$ is said to be proper if it is non empty and differs from the entire lattice $L$. If the lattice $L$ is a bounded lattice, then a filter is proper if and only if it does not contains $\mathbb{0}$.

An ideal of $L$ is a subset $I$ of $L$ such that

I1 If $x \in I$ and $y \in I$, then $x \curlyvee y \in I$;

I2 if $x \in I$, then, for every $y \in L$ such that $y \preceq x, y \in I$.

An ideal of a lattice $L$ is said to be proper if it differs from the entire lattice $L$. If the lattice $L$ is a bounded lattice, then an ideal is proper if and only if it does not contains $\mathbb{1}$. 
Given a subset $A$ of a lattice $L$, one can always find the $\subseteq$-smaller filter among all filters of $L$ that contains $A$ as subset (this collection is obviously not empty for $L$ is listed among its elements) which will be denoted by $\mathbb{P}(A)$. It's easy to see that

$$
\mathbb{P}(A)=\left\{x \in L: \exists A_{0}\left(A_{0} \in[A]^{<\omega} \backslash\{\emptyset\} \wedge \sup A_{0} \preceq x\right)\right\} .
$$

The set $\mathbb{P}(A)$ is called filter generated by $A$. Analogously, given a subset $A$ of a lattice $L$, one can always find the $\subseteq$-smaller ideal among all ideal of $L$ (this collection is obviously

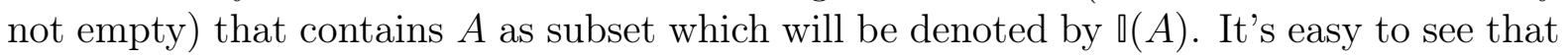

$$
\llbracket(A)=\left\{x \in L: \exists A_{0}\left(A_{0} \in[A]^{<\omega} \backslash\{\emptyset\} \wedge x \preceq \inf A_{0}\right)\right\} .
$$

The set $\llbracket(A)$ is called ideal generated by $A$.

Within a bounded lattice $L$, a subset $A$ of $L$ has the finite intersection property, abbreviated as FIP, if any finite subset of $A$ has a non $\mathbb{O}$ infimum. One can prove that $A$ has the FIP if and only if the generated filter $\mathbb{P}(A)$ is proper.

A filter $F$ of a lattice $L$ such that, for every elements $x$ and $y$ of $R$ whose supremum is inside $F, x \in F$ or $y \in F$ is called a prime filter of $R$. The property of being prime will be the core of the work done the the part II of the present account.

2.3.1 Lemma. If $P$ is a prime filter of a bounded distributive lattice $L$, then $L \backslash P$ is an ideal of $L$.

2.3.2 Theorem. Let $L$ be a distributive bounded lattice, $I$ an ideal of $L$ and $F$ a proper filter of $L$ that does not meet $I$. Then, there is a prime filter $P$ of $L$ that contains $F$ and does not meet $I$.

Proof: Consider the collection $\mathscr{F}$ of all proper filters of $L$ that contain $F$ and do not meet $I$. For the condition $I \cap F=\emptyset$, the collection $\mathscr{F}$ is not empty. Moreover, let $\mathcal{L} \subseteq \mathscr{F}$ be any totally ordered by $\subseteq$, then $\bigcup \mathcal{L}$ is a member of $\mathscr{F}$ that majorates every member of $\mathcal{L}$. Thus, by the Kuratowski-Zorn Lemma, $\mathscr{F}$ has a $\subseteq$-maximal member $P$.

Now, let $x$ and $y$ be elements of $L$ such that $x \notin P$ and $x \curlyvee y \in P$. Then, the filter generated by $P \cup\{x\}$ must meet the idean $I$, i.e. there is $p_{0} \in P$ such that $x \curlywedge p_{0} \in I$, for $P$ is maximal. Now, suppose that $y \notin P$; then, with the same argument, there is a $p_{1} \in P$ such that $y \curlywedge p_{1} \in I$. Thus, as $I$ is an ideal of $L,\left(x \curlywedge p_{0}\right) \curlyvee\left(y \curlywedge p_{1}\right) \in I$. But, as $L$ is a distributive lattice,

$$
\left(x \curlywedge p_{0}\right) \curlyvee\left(y \curlywedge p_{1}\right)=(x \curlyvee y) \curlywedge\left(y \curlyvee p_{0}\right) \curlywedge\left(x \curlyvee p_{1}\right) \curlywedge\left(p_{0} \curlyvee p_{1}\right) \in P,
$$

which is an absurd. Thus, $y \in P$, which testifies that $P$ is an prime filter of $L$.

A $\subseteq$-maximal proper filter of a distributive bounded lattice is called ultrafilter. The collection of all ultrafilters of $L$ will be denoted by $\operatorname{stn} L$ By definition an ultrafilter is always not empty. An usual application of Kuratowski-Zorn's Lemma (similiar to the above) yelds

2.3.3 Theorem. Let $L$ be a distributive bounded lattice. Then, any subset $A$ of $L$ having the FIP is inscribed within an ultrafilter of $R$. 
2.3.4 Lemma. Let $P$ be a filter of a lattice $L$. Then, in order to $P$ be an ultrafilter is necessary and sufficient that, for every $x \in R \backslash P$, there is a $y \in P$ testifying that $x \curlywedge y=\mathbb{0}$.

Proof: Suppose that $P$ is an ultrafilter; then $P \cup\{x\}$ does not have the FIP, because $P$ is maximal. Thus, there is a $y \in P$ such that $x \curlywedge y=\mathbb{O}$.

For the converse, let $F$ be a filter of $L$ such that $P \subseteq F$. If is not true that $P$ and $F$ are equal, than there must be a element $x \in F \backslash P$ that, by the hypothesis, meets some $y \in P$ at their infimum $\mathbb{O}$. Hence, $F$ is not proper.

2.3.5 Corolary. For every two distinct ultrafilter $P$ and $Q$ of a bounded lattice $L$, is possible to find $x \in P$ and $y \in Q$ such that $x \curlywedge y=\mathbb{0}$.

\subsection{Boolean Algebras}

In this section, the basic theory of Boolean algebras will be presented.

2.4.1 Lemma. [Nac47] Let $L$ be a distributive and bounded lattice in which all proper prime filters are ultrafilters. Then, given an element $x$ of $L$, there is an unique element $y$ of $L$ such that

$$
\mathbb{O}=x \curlywedge y \quad \text { and } \quad x \curlyvee y=\mathbb{1} \text {. }
$$

Proof: Such element $y$ in the statement of the present lemma will be called Complementation of $x$. The distributiveness of $L$ implies that, when it exists, the complementation $y$ of an element $x$ is unique. Indeed, if $y^{\prime}$ is any complementation of $x$, then

$$
\begin{aligned}
y & =y \curlywedge \mathbb{1}=y \curlywedge\left(y^{\prime} \curlyvee x\right)=\left(y \curlywedge y^{\prime}\right) \curlyvee(y \curlywedge x)=\left(y \curlyvee y^{\prime}\right) \curlyvee \mathbb{O}=y \curlywedge y^{\prime}= \\
& =\left(y \curlywedge y^{\prime}\right) \curlyvee\left(y^{\prime} \curlywedge x\right)=(y \curlyvee x) \curlywedge y^{\prime}=\mathbb{1} \curlywedge y^{\prime}=y^{\prime} .
\end{aligned}
$$

Now, if there is a $x \in L$ that does not have a complementation, then

$$
F_{0}=\{z \in L: z \curlyvee x=\mathbb{1}\} \text { and } F_{1}=\left\{z \in L: \exists y\left(y \in F_{0} \wedge(y \curlywedge x \leq z)\right)\right\} .
$$

are proper filters of $L$. By 2.3 .3 (pag.11), there is an ultrafilter, and thus a prime filter $P$ of $L$ that contains $F_{1}$. Let $I$ be the ideal generated by $(L \backslash P) \cup\{x\}$. If it is possible to find a $z \in F_{0} \cap I$, then the equality GI (pag.11) yields an element $y$ of $L \backslash P$ such that $z \leq y \curlyvee x$. But, as $z$ is also an element of $F_{0}$, one has

$$
\mathbb{1}=z \curlyvee x \leq y \curlyvee x,
$$

which proves that $y \in F_{0} \subseteq F_{1} \subseteq P$, whose absurdity is clear. Therefore, the theorem 2.3 .2 (pag.11) testifies the existence of a prime filter $Q$ of $L$ such that $F_{0} \subseteq Q$ and $Q \cap I=\emptyset$. By the clause $x \in I$, one has

$$
Q \subseteq L \backslash I \subset P,
$$


which proves that $Q$ cannot be an ultrafilter. Thus, the assumption of that $x$ is an element of $L$ without complement is incorrect.

As for the lattice $L$ of the lemma 2.4 .1 (pag.12), one can define a function $(\cdot)^{\star}: L \rightarrow$ $L$ such that, for every $x \in L, x^{\star}$ is the complement of $x$ in $L$. A bounded distributive lattice $B$ whereas every element has a complement (or, equivalently, every prime filter is an ultrafilter) is called Boolean algebra.

2.4.2 Lemma. (De Morgan) Given elements $x$ and $y$ of a Boolean algebra $B$,

$(2.4 .2 .1) . x \curlywedge x^{\star}=\mathbb{O}$ and $x \curlyvee x^{\star}=\mathbb{1}$;

$(2.4 .2 .2) \cdot\left(x^{\star}\right)^{\star}=x$

$(2.4 .2 .3) .(x \curlywedge y)^{\star}=x^{\star} \curlyvee y^{\star}$; and

$(2.4 .2 .4) .(x \curlyvee y)^{\star}=x^{\star} \curlywedge y^{\star}$.

2.4.3 Lemma. Let $\langle L,+, \cdot, \mathbb{O}, \mathbb{1}\rangle$ be an algebraic structure in the fashion of 2.2.3 (pag.9) that also has an unary operation $(\cdot)^{\bullet}: L \rightarrow L$ satisfying

(BA) $x \curlywedge x^{\bullet}=\mathbb{0}$

Then $L$ is a Boolean algebra with $\curlyvee=+, \curlywedge=\cdot, \star=\bullet$ and whose order is given by one of the equivalent relations of 2.2.2 (pag.8).

\subsection{Examples}

2.5.1 Example. Let $L=] 0,1[$ with the usual order inherited from $\mathbf{R}$. Then, the trichotomy property testifies that

$$
x \curlywedge y=\min \{x, y\} \quad \text { and } \quad x \curlyvee y=\max \{x, y\} .
$$

This lattice is distributive, but the Archimedian property of the real line proves that $L$ is not bounded.

2.5.2 Example. In the set of all positive natural numbers $\mathbf{N}_{>0}$, let $\preceq$ be the relation defined by $m \preceq n$ if and only if $m$ divide $n$, i.e. there is a $k \in \mathbf{N}$ such that $n=k \cdot m$. It is easy to check that this is a partial order on $\mathbf{N}_{>0}$. Moreover, for any positive natural numbers $m$ and $n$, one can observe that

$$
m \curlywedge m=\operatorname{gcd}(m, n) \text { and } m \curlyvee n=\operatorname{lcm}(m, n),
$$

where $\operatorname{gcd}(m, n)$ is the greatest common divisor of $m$ and $n$, while $\operatorname{lcm}(m, n)$ is the least common multiple of $m$ and $n$.

This lattice is bound from below by $\min \mathbf{N}_{>0}=1$. If $\left\langle\mathbf{p}_{n}\right\rangle$ is the enumeration of $\mathbf{P}$ that conserves the order of $\mathbf{P}$, then the Fundamental Theorem of Arithmetics states that for any positive natural number $n$, there exists a unique sequence $\alpha(n) \in^{\mathbf{N}} \mathbf{N}$ such that 
(1.) $\left|\left\{k \in \mathbf{N}: \alpha(n)_{k} \neq 0\right\}\right|<\omega$;

(2.) $n=\prod_{k \geq 0} \mathbf{p}_{k}^{\alpha(n)_{k}}$.

Thus,

$$
\operatorname{lcm}(m, n)=\prod_{n \geq 0} \mathbf{p}_{k}^{\max \left\{\alpha(m)_{k}, \alpha(n)_{k}\right\}} \quad \text { and } \quad \operatorname{gcd}(m, n)=\prod_{n \geq 0} \mathbf{p}_{k}^{\min \left\{\alpha(m)_{k}, \alpha(n)_{k}\right\}}
$$

Therefore, for any positive natural numbers $a$ and $b$,

$$
\alpha(a \curlyvee b)=\max \{\alpha(a), \alpha(b)\} \quad \text { and } \quad \alpha(a \curlywedge b)=\min \{\alpha(a), \alpha(b)\} ;
$$

hence, given a positive natural number $c$,

$$
\begin{aligned}
\alpha(a \curlywedge(b \curlyvee c)) & =\min \{\alpha(a), \max \{\alpha(b), \alpha(c)\}\}=\max \{\min \{\alpha(a), \alpha(b)\}, \min \{\alpha(a), \alpha(c)\}\}= \\
& =\alpha((a \curlywedge b) \curlyvee(a \curlywedge c)) .
\end{aligned}
$$

As $\alpha$ is injective (as a function from $\mathbf{N}_{>0}$ to ${ }^{\mathbf{N}} \mathbf{N}$ ), $\left\langle\mathbf{N}_{>0}, \mid\right\rangle$ is a distributive lattice.

2.5.3 Example. For any set $X, \wp(X)$ is a lattice where the order is $\subseteq$; for any non-empty $\mathcal{A} \subseteq \wp(X)$

$$
\inf \mathcal{A}=\bigcap \mathcal{A} \text { and } \sup \mathcal{A}=\bigcup \mathcal{A}
$$

in particular, for any subsets $A$ and $B$ of $X$,

$$
A \curlywedge B=A \cap B \text { and } A \curlyvee B=A \cup B .
$$

This lattice is distributive and is obviously bounded by $\emptyset$ and $X$.

In the example immediately above, any non-empty subset of $X$ has a supremum and an infimum; lattices of this sort will be called complete lattices. The lattice from the example 2.5.1 (pag.13) is not complete.

Hasse diagrams provide a way to represent finite lattices in a graphic fashion:

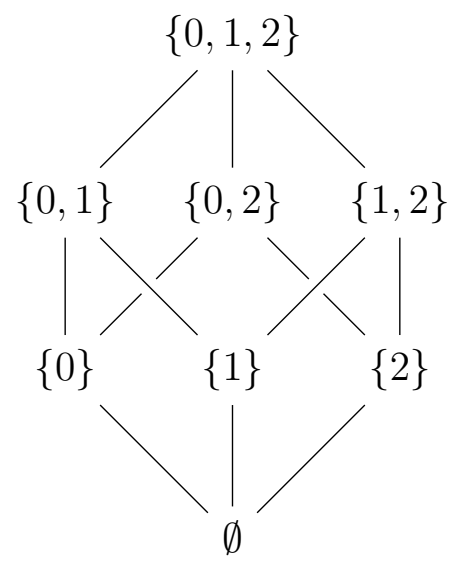


In the figure above, the lattice $\wp(\{0,1,2\})$ is represented. The comparison between to nodes takes advantages of the associativiness of the ordering and can be derived from the existence of a path between these nodes; a node $x$ is greater than a node $y$ if $x$ is higher in the path that connects these two nodes. Therefore, $\emptyset \subseteq\{0,1\}$, while $\{0,1\}$ and $\{1,2\}$ are not comparable. The infimum of two elements can be obtained by finding the intersection of the paths descending from theses two elements, and the supremum can be obtained by finding the intersection of the paths ascending from theses two elements.

2.5.4 Example. Let $\{0,1,2,3,4\}$ with the order expressed by the following Hasse diagram:

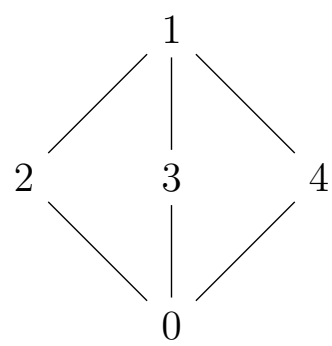

Then,

$$
2 \curlywedge(3 \curlyvee 4)=2 \curlywedge 1=2,
$$

but

$$
(2 \curlywedge 4) \curlyvee(2 \curlywedge 3)=0 \curlyvee 0=0 .
$$

is called the diamond lattice and is not distributive, as it is shown above. There is another famous non-distributive lattice called the pentagon lattice, whose Hasse diagram is

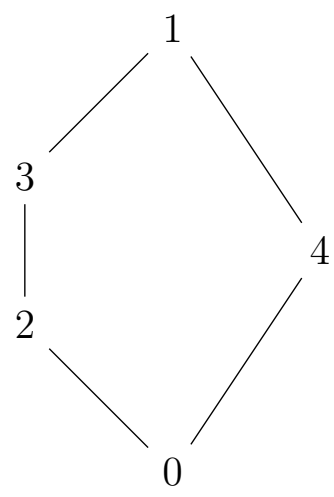

It can be shown that a lattice $L$ is distributive if and only if neither the diamond nor the pentagon lattice can be embedded in $L$ as a sublattice - a sublattice of a (bounded) lattice $L$ is a subset $R$ of $L$ that also is a (bounded) lattice with respect to the inherited order from $L$ -

2.5.5 Example. For any set $X$, the collection $\mathcal{T}$ of all possibles topologies over $X$ is non-empty, as it has $\wp(X)$ and $\{\emptyset, X\}$ among its elements. Moreover, given any non empty sub-collection $\mathcal{T}_{0} \subseteq \mathcal{T}$, the intersection $\bigcap \mathfrak{T}$ is also a topology over $X$. Thus, $\mathcal{T}$ is a complete lattice with respect of $\subseteq$, where, for any non-empty $\mathcal{J}_{0} \subseteq \mathcal{T}$,

$$
\inf \mathscr{T}_{0}=\bigcap \mathcal{T}_{0} \text { and } \sup \mathcal{T}_{0}=\bigcap\left\{\tau \in \mathscr{T}_{0}: \bigcup \mathcal{T}_{0} \subseteq \tau\right\}
$$


2.5.6 Example. Let $X$ be a topological space, in which the topology is $\tau_{X}$, and let $C$ be the collection of all closed subsets of $X$. Then, $\tau_{X}$ and $C$ are complete lattices with respect of $\subseteq$; in fact given any non empty collection $\mathcal{A}$ of open subsets of $X$,

$$
\inf \mathcal{A}=\bigcup\{U \in \tau: U \subseteq \bigcap \mathcal{A}\} \quad \text { and } \sup \mathcal{A}=\bigcup \mathcal{A}
$$

if $\mathscr{F}$ is a non empty collection of closed subsets of $X$,

$$
\inf \mathscr{F}=\bigcap F \text { and } \sup \mathscr{F}=\bigcap\{F \in C: \bigcup \mathscr{F} \subseteq F\}
$$




\section{CHAPTER 3}

\section{Topological Spaces and their morphisms}

\subsection{Neighbourhood and bases}

Most of the topological concepts used in the present work will be as it is in [Eng89]. For any topological space $X$, unless the inverse is stated, topology of $X$ will be represented as $\tau_{X}$. Since this is not an introductory text in topology, is expected from the reader a basic knowledge of topology for which this chapter is merely a quickly reminder.

For any subset $A$ of a topological space $X$, the interior of $A$ in $X$ is the $\subseteq$-supremum of all open subsets of $X$ that is contained in $A$ and will be denoted by $\operatorname{int}_{X}(A)$; the $\subseteq$ infimum of all closed subsets of $X$ that contain $A$ as a subset is called closure of $A$ in $X$ and is denoted by $\mathrm{cl}_{X}(A)$. Evidently,

$$
\operatorname{int}_{X} A=\bigcup\left\{U \in \tau_{X}: U \subseteq A\right\}
$$

and

$$
\mathrm{cl}_{X} A=\bigcap\left\{F \subseteq X:\left(X \backslash F \in \tau_{X}\right) \wedge(A \subseteq F)\right\} .
$$

A countable union of closed subsets of a topological space $X$ is called $F_{\sigma}$ set. A countable intersection of open subsets of $X$ is called $G_{\delta}$ set.

A dense subset of a topological space $X$ is any $D \subseteq$ whose clousure is $X$.

Let $X$ be a topological space; given a point $x$ of $X$, any open set $U$ of $X$ containing $x$ is said to be a neighbourhood of $x$ in $X$. $V_{X}(x)$ will denote the collection of all neighbourhoods of $x$ in $X$.

Any collection $\mathscr{B}$ of open subsets of a topological space $X$ satisfying

BO1 Given any $x \in X$, there is an $U \in \mathcal{B}$ such that $x \in U$; and 
BO2 given $U_{0}, U_{1} \in \mathscr{B}$ such that one can find a $x \in U_{0} \cap U_{1}$, then one can find a $U \in \mathscr{B}$ such that $x \in U \subseteq U_{0} \cap U_{1}$.

is called a base of open sets for the space $X$. It is easy to see that if $\mathcal{G}$ is any collection of subsets inscribed in a set $Y$ fulfilling BO1 and BO2, then

$$
\tau=\{\bigcup \mathcal{A}: \mathcal{A} \subseteq \mathcal{O}\}
$$

is a topology for some topology $\tau$ for $Y$ such that $\mathcal{O}$ is a base of open sets for $\langle Y, \tau\rangle$.

Analogously, a collection $\mathscr{F}$ of closed subsets inscribed in space $X$ satisfying

BF1 Given any $x \in X$, there is a $F \in \mathscr{F}$ such that $x \notin F$; and

BF2 given $F_{0}, F_{1} \in \mathscr{B}$ such that there is a point $x \in X$ satisfying $x \notin F_{0} \cup F_{1}$, then one can find a $F \in \mathscr{B}$ such that $x \notin F$ and $F_{0} \cup F_{1} \subseteq F$.

is called a base of closed sets for the space $X$. Evidently, if $\mathscr{D}$ is any collection of subsets circumscribed by a set $Y$ that fulfills BF2 and BF2, then $\mathscr{B}=\{X \backslash F: F \in \mathscr{D}\}$ is a base of open sets for some topology $\tau$ for $Y$; thus, one can construct the topology a $\tau$ for a set $Y$ by finding a $\mathscr{D} \subseteq \wp(X)$ that fulfills BF2 and BF2, whose base for closed subsets will be $\mathscr{D}$.

A subbase for a topological space $X$ is a collection $\mathscr{P} \subseteq \tau_{X}$ such that

$$
\mathscr{B}_{\mathscr{P}}=\left\{\bigcap \mathscr{P}_{0}: \mathscr{P}_{0} \in[\mathscr{P}]^{<\omega} \backslash\{\emptyset\}\right\}
$$

is a base of open sets for $X$. Evidently $\bigcup \mathscr{P}=X$; moreover, if $\tau$ is any topology for $X$ that contains $\mathscr{P}$ as a subset, then $\tau_{X} \subseteq \tau$. Reciprocally, any collection $\mathcal{A}$ whose union is $X$ is a subbase of some topology for $X$; and if $\tau$ is the $\subseteq$-smallest topology for $X$ containing $\mathcal{A}$ as a subset, then $\mathcal{A}$ must be a subbase for $\tau$.

\subsection{Continuous Functions}

Let $X$ and $Y$ be any topological spaces. A function $f: X \rightarrow Y$ is called continuous if fulfills any of the following equivalent conditions [Eng89]:

CF1 The inverse image under $f$ of any open subset of $Y$ is an open subset of $X$;

CF2 the inverse image under $f$ of any member of a subbase for $\tau_{Y}$ is an open subset of $X$;

CF3 the inverse image under $f$ of any member of a base of open sets for $\tau_{Y}$ is an open subset of $X$;

CF4 the inverse image under $f$ of any closed subset of $Y$ is a closed subset of $X$; 
CF5 the inverse image under $f$ of any member of a base of closed sets for $\tau_{Y}$ is a closed subset of $X$;

CF6 for any $A \subseteq X, f\left[\operatorname{cl}_{X} A\right] \subseteq c l_{Y} f[A]$; and

CF7 for any $B \subseteq Y, \mathrm{cl}_{X} f^{-1}[B] \subseteq f^{-1}\left[\mathrm{cl}_{Y} B\right]$.

If there is a continuous surjection from a topological space $X$ onto a topological space $Y$, then $Y$ is said to be a continuous image of $X . X$ and $Y$ are said to be homeomorphic if there is a continuous bijection from $X$ onto $Y$ whose the inverse is also continuous; in this case, $f$ is said to be a homeomorphism.

Let $X$ be any set and $\left\langle Y_{s}\right\rangle_{s \in S}$ a collection of topological spaces; for any $s \in S$, suppose available a function $f_{s}: X \rightarrow Y_{s}$; for any $s \in S$, let $\tau_{s}$ be the topology of $Y_{s}$. Then it is possible to define a topology $\tau$ for $X$ such that

(i) any function from the collection $\left\langle f_{s}\right\rangle_{s \in S}$ is continuous; and

(ii) any topology $\tau^{\prime}$ for $X$ that witnesses the continuity of any function from $\left\langle f_{s}\right\rangle_{s \in S}$ must contains $\tau$, i.e. any open set of $\langle X, \tau\rangle$ must be open in $\left\langle X, \tau^{\prime}\right\rangle$

Indeed, $\wp(X)$ testify that the set of all topologies for $X$ that make continuous each function from $\left\langle f_{s}\right\rangle_{s \in S}$ is not empty; thus, being $\tau$ the intersection of all these topologies, the result follows. Moreover, $\bigcup_{s \in S}\left\{f_{s}^{-1}[V]: V \in \tau_{Y_{s}}\right\}$ is a subbase for $\langle X, \tau\rangle^{1}$.

\subsection{Axioms of separation}

A topological space $X$ is said to be a $T_{1}$ space if meets the following equivalent conditions

$\mathbf{T}_{1}$ given a pair of distinct points $x$ and $y$ within $X$, there are open subsets $U$ and

$V$ of $X$ such that $U \cap V$ does not contain these two points;

$\mathbf{T}_{1}^{\prime}$ for any $x \in X,\{x\}=\bigcap \mathcal{V}_{X}(x)$; and

$\mathbf{T}_{1}^{\prime \prime}$ for any $x \in X$, the singleton $\{x\}$ is closed in $X$.

More important is the class of Hausdorff spaces whose members are asked to separate any two distinct points by disjoint open subsets; i.e. a topological space $X$ is a Hausdorff space if and only if given any distinct points $x$ and $y$ of $X$, one can find disjoint open subsets $U$ and $V$ of $X$ such that $x \in U$ and $y \in V$. Evidently, any Hausdorff space is a $T_{1}$ space.

It is clear from the definition of the class of all Hausdorff spaces that one could select the open subsets that separates points from a base for the topological space in question (actually, this condition is equivalent to the axiom that defines Hasudorff spaces); but one cannot replace base by a subbase as any set $X$ with only four elements and at which

\footnotetext{
${ }^{1}$ The lemma 3.6.1 will explain the reason for take this set as subbase instead of base.
} 
one defines the Hausdorff topology $[X]^{\leq 2} \cup\{X\}$ has $\mathscr{P}=[X]^{2}$ as a subbase that does not separates points by disjoint open sets.

If $f$ and $g$ are two continuous functions from a topological space $X$ to a Hausdorff space $Y$, then

$$
F=\{x \in X: f(x)=g(x)\}
$$

is a closed subset of $Y$. Thus, two continuous functions whose ranges is a Hausdorff space and coincide over a dense subset must be equal.

A $\mathrm{T}_{1}$ space $X$ in which any point not belonging to a closed subset of $X$ can be separated from this particular closed subset by disjoint open subsets of $X$ is said to be a regular space; i.e. a $\mathrm{T}_{1}$ space $X$ is regular if, given a point $x$ of $X$ and a closed subset $F$ of $X$ satisfying $x \notin F$, one can find disjoint open subsets $U$ and $V$ of $X$ such that $x \in U$ and $F \subseteq V$.

3.3.1 Theorem. A $\mathrm{T}_{1}$ topological space is regular if and only if for every non empty open subset $V$ of $X$ and $x \in V$, an open subset $U$ of $X$ such that $x \in U \subseteq \operatorname{cl}_{X} U \subseteq V$.

Proof: Indeed, for some non empty $V$, given an $x \in V$, one can find open and disjoint subsets $U$ and $W$ of $X$ such that $x \in U$ and $X \backslash V \subseteq W$; thus

$$
U \subseteq X \backslash W \subseteq V
$$

which proves that $\operatorname{cl}_{X} U \subseteq V$.

For the converse, if $x$ is a point of $X$ that is not listed among the elements of some closed subset $F$ of $X$, then one can find a open set $U$ such that $x \in U \subseteq \operatorname{cl}_{X} U \subseteq X \backslash F$; taking $W=X \backslash \mathrm{cl}_{X} U$, one can easily verify that $x \in U, F \subseteq W$ and $\bar{U} \cap W=\bar{\emptyset}$.

In a topological space $X$, a set $F$ is called functionally closed (also called zero set) if there is a continuous function $f: X \rightarrow \mathbf{I}$ such that $F=f^{-1}[\{0\}]$. As $\mathbf{I}$ is a Hausdorff space, any functionally closed subset of $X$ is closed. Moreover, given a sequence $\left\langle f_{n}\right\rangle$ of continuous functions from $X$ to $\mathbf{I}, f(x)=\sum_{n>0} 2^{-n} f_{n}$ defines a continuous function, thus intersection of any non empty countable family of functionally closed subsets of $X$ is a functionally closed subset of $X$. The product of two continuous being a continuous function proves that finite union of functionally closed subsets is also a functionally closed subset of $X$. The collection of all functionally closed subsets of $X$ shall be denoted as $\mathrm{FC}(X)$. Hence, $\mathrm{FC}(X)$ is a sublattice of the lattice composed by all closed subsets of $X$.

In a topological space $X$, a set $U$ is said to be a functionally open subset (also called cozero set) of $X$ if its complement is functionally closed. Clearly, any functionally open of $X$ is an open subset of $X$, finite intersections of functionally open subsets of $X$ is a functionally open subset of $X$ and countable union of functionally open subsets of $X$ is a functionally open subsets of $X$. The collection of all functionally open subsets of $X$ shall be denoted as $\mathrm{FO}(X)$. Hence, $\mathrm{FO}(X)$ is a sublattice of the lattice composed by all open subsets of $X$.

If $F_{0}$ and $F_{1}$ are disjoint functionally closed subsets of a topological space $X$, let, for every $i \in\{0,1\}, f_{i}: X \rightarrow \mathbf{I}$ be a continuous functions such that $F_{i}=f_{i}^{-1}[\{0\}]$. Then 
$f: X \rightarrow \mathbf{I}$ given by

$$
f(x)=\frac{f_{0}(x)}{f_{0}(x)+f_{1}(x)}
$$

is a continuous function satisfying, for any $i \in\{0,1\}, F_{i}=f^{-1}[\{i\}]$.

Two subsets $A$ and $B$ of a topological space $X$ are said to be completely separated if there is a continuous function $g: X \rightarrow \mathbf{I}$ such that $A \subseteq f^{-1}[\{0\}]$ and $B=g^{-1}[\{1\}]$. Obviously, two subsets $A$ and $B$ are completely separated if and only if one can find two disjoint functionally closed subsets $F$ and $G$ of $X$ such that $A \subseteq F$ and $B \subseteq G$.

Any $\mathrm{T}_{1}$ space $X$ admitting a base of open sets formed by functionally open subsets of $X$ is called a Tychonoff space. Equivalently, the class of Tychonoff spaces can be characterized by separating a point of $X$ that does not belong to some closed subset $F$ of $X$ by functionally closed subsets of $X$; i.e. for every $x \in X$ and every closed subset $F$ of $X$ such that $x \notin X$, there is a continuous function $f: X \rightarrow \mathbf{I}$ satisfying $f(x)=0$ and $F \subseteq f^{-1}[\{1\}]$.

In a $\mathrm{T}_{1}$ space $X$, a base open sets $\mathscr{B}$ for $X$ is called a normal base of open subsets [Fri64; Zaı̆67] for $X$ if

(NBO1) for every $x \in X$ and any $U \in \mathscr{B}$ containing $x$, one can find a $V \in \mathscr{B}$ that does not contains $x$ and $U \cup V=X$;

(NBO2) given any elements $U$ and $V$ of $\mathscr{B}$ such that $U \cup V=X$, there are disjoint $U_{0}$ and $V_{0}$ listed among the elements of $\mathscr{B}$ such that $X \backslash U \subseteq U_{0}$ and $X \backslash V \subseteq V_{0}$.

Analogously, one can define the notion of normal base of closed subsets for $X$, as a base for closed subsets $\mathscr{Z}$ such that

(NBC1) given any $x \in X$ and any set closed subset $F$ of $X$ that has not $x$ as its element, there is a member of $\mathscr{Z}$ that contains $x$ and does not meet $F$; and

(NBC2) given two disjoint elements $F$ and $G$ of $\mathscr{Z}$, there are members $F^{\prime}$ and $G^{\prime}$ of $\mathscr{L}$ whose complements are disjoint, $F \subseteq X \backslash F^{\prime}$ and $G \subseteq X \backslash G^{\prime}$.

3.3.2 Theorem. A $\mathrm{T}_{1}$ space $X$ has a normal base for open subsets (or, equivalently, a normal base for closed subsets) if and only if $X$ is a Tychonoff space.

Proof: If $X$ is a Tychonoff space, then $\mathrm{FO}(X)$ is a normal base for open subsets.

If $X$ is a $\mathrm{T}_{1}$ space with a normal base of open sets $\mathcal{B}$, given any point $x \in X$ and any open set $U \in \mathscr{B}$ containing $x$, there is a $V \in \mathscr{B}$ such that

$$
x \in V \subseteq \mathrm{cl}_{X} V \subseteq U .
$$

In particular, if $F$ is a closed subset of $X$ that does not contains $x$, then is possible to find a $U_{1} \in \mathscr{B}$ such that $x \in U_{0} \subseteq X \backslash F$. Let $\left\langle r_{n}\right\rangle$ be an enumeration of all rational numbers contained inside $\mathbf{I}$ such that $r_{0}=1$. Suppose that, for a natural number $n$ and any $m \leq n$, there is a $V_{m} \in \mathscr{B}$ such that, given any $k \leq n$,

$$
x \in V_{r_{k}} \subseteq \operatorname{cl}_{X} V_{r_{k}} \subseteq U_{r_{m}}
$$


if and only if $r_{k}<r_{l}$. Let $\rho_{n}$ be the smallest among $r_{0}, \ldots r_{n}$ that is greater than $r_{n+1}$. Then, one can find an $U_{r_{n+1}} \in \mathcal{B}$ such that $x \in U_{r_{n+1}}$ and, for any $m, k \leq n+1$

$$
\mathrm{cl}_{X} U_{r_{k}} \subseteq U_{r_{m}}
$$

if and only if $r_{k}<r_{m}$. Thus, the collection $\left\langle U_{r}\right\rangle_{r \in \mathbf{I} \cap \mathbf{Q}}$ satisfies, for any rationals $r_{0}, r_{1} \in \mathbf{I}$,

$$
\operatorname{cl}_{X} U_{r_{0}} \subseteq U_{r_{1}} \text { if and only if } r_{0}<r_{1} .
$$

Define $f: X \rightarrow \mathbf{I}$ as

$$
f(z)= \begin{cases}\inf \left\{r \in \mathbf{Q} \cap \mathbf{I}: z \in U_{r}\right\}, & \text { if } z \in U_{1} ; \text { or } \\ 1, & \text { if } z \notin U_{1}\end{cases}
$$

Evidently $f(x)=0$ and $f[F] \subseteq 1$. Now, for any $0 \leq a \leq 1, f(x)<a$ if and only if there is a rational $r \in \mathbf{I}$ such that $r<a$ and $z \in U_{r}$. Thus,

$$
f^{-1}\left[\left[0, a[]=\bigcup_{r \in \mathbf{Q} \cap[0, a[} U_{r}\right.\right.
$$

If $f(z)>a$ occurs, then $(\star)$ witnesses the existence of a rational number $r \in \mathbf{I}$ such that $r>b$ and $z \notin c l_{X} U_{r}$. Thus,

$$
\left.\left.f^{-1}[] a, 1\right]\right]=\bigcup_{r \in \mathbf{Q} \cap] a, 1]}\left(X \backslash \operatorname{cl}_{X} V_{r}\right)=X \backslash \bigcap_{r \in \mathbf{Q} \cap a, 1]} \operatorname{cl}_{X} V_{r}
$$

As

$$
\{[0, a[: a \in \mathbf{I}\} \cup\{] a, 1]: a \in \mathbf{I}\}
$$

is a base for $\mathbf{I}, f$ must be continuous.

A normal space is a $\mathrm{T}_{1}$ space in which is possible to separate disjoint closed subsets by disjoint open subsets. Clearly, a $\mathrm{T}_{1}$ space is normal if and only if, for any closed subset $F \subseteq X$ and any open $U$ of $X$ containing $F$, one can find a open set $V$ of $X$ such that

$$
F \subseteq V \subseteq \operatorname{cl}_{X} V \subseteq U
$$

Actually, one can prove that

3.3.3 Lemma. A $\mathrm{T}_{1}$ space $X$ in which, for every closed set $F$ of $X$ and open set $V$ of $X$ that contains $F$, for any $n \in \mathbf{N}$, one can find a open set $V_{n}$ of $X$ such that

$$
F \subseteq \bigcup_{n \in \mathbf{N}} V_{n} \quad \text { and } \quad \mathrm{cl}_{X}\left(V_{n}\right) \subseteq V
$$

Proof: Let $F$ and $G$ be two closed and mutually disjoint sets contained in $X$. By letting $V=X \backslash G$ and $U=X \backslash F$, the hypothesis produces, for any natural number $n$, open subsets $U_{n}$ and $V_{n}$ of $X$ such that

$$
F \subseteq \bigcup_{n \in \mathbf{N}} V_{n} \quad \text { and } \quad \operatorname{cl}_{X}\left(V_{n}\right) \subseteq V
$$


and

$$
G \subseteq \bigcup_{n \in \mathbf{N}} U_{n} \quad \text { and } \quad \operatorname{cl}_{X}\left(U_{n}\right) \subseteq U
$$

For any $n \in \mathbf{N}$, let

$$
U_{n}^{\prime}=U_{n} \backslash \bigcup_{m \leq n} \operatorname{cl}_{X}\left(V_{n}\right) \quad \text { and } \quad V_{n}^{\prime}=V_{n} \backslash \bigcup_{m \leq n} \operatorname{cl}_{X}\left(U_{n}\right)
$$

A simple induction argument shows that, for each $n \in \mathbf{N}, U_{n}^{\prime}$ and $V_{n}^{\prime}$ are mutually disjoint open subsets of $X$. Thus, the unions $\bigcup_{n \in \mathbf{N}} U_{n}^{\prime}$ and $\bigcup_{n \in \mathbf{N}} V_{n}^{\prime}$ are mutually disjoint open subsets of $X$ that contains $G$ and $F$, respectively.

3.3.4 Lemma. [Ury25] A $\mathrm{T}_{1}$ space is normal if and only if disjoint closed subsets in this space are completely separated.

Proof: Evidently, a $T_{1}$ space in which disjoint closed subsets are completely separated is normal. Conversely, let $X$ be a normal space and $F, G \subseteq X$ be two disjoint closed subsets of $X$. If $V_{1}=X \backslash G$, then the normality of $X$ witnesses the existence of an open subset $V_{0}$ of $X$ such that

$$
F \subseteq V_{0} \subseteq \mathrm{cl}_{X} V_{0} \subseteq V_{1}
$$

Let $\left\langle r_{n}\right\rangle$ be an enumeration of all rational numbers inside $\mathbf{I}$ such that $r_{0}=0$ and $r_{1}=1$. For any $n \geq 2$, suppose that are available, for any $m \leq n$, an open set $U_{r_{m}}$ such that, for any $k \leq n, \operatorname{cl}_{X} U_{r_{k}} \subseteq U_{r_{m}}$ if and only if $r_{k}<r_{m}$. The enumeration $\left\langle r_{n}\right\rangle$ ensures the existence of a $\lambda_{n}$ that is the greatest among $r_{0}, \ldots, r_{n}$ that is smaller than $r_{n+1}$; similarly, one can find the smallest $\rho_{n}$ among $r_{0}, \ldots, r_{n}$ that is greater than $r_{n+1}$; evidently, $\lambda_{n}<\rho_{n}$. As the closed set $\mathrm{cl}_{X} V_{\lambda_{n}}$ is a subset of the open set $V_{\rho_{n}}$, the normality of $X$ witnesses the existence of an open subset $V_{r_{n+1}}$ of $X$ such that $\mathrm{cl}_{X} U_{r_{k}} \subseteq U_{r_{m}}$ if and only if $r_{k}<r_{m}$, whenever $k, m \leq n+1$. Thus, by induction, is possible to define a collection $\left\langle V_{r}\right\rangle_{r \in \mathbf{I} \cap \mathbf{Q}}$ such that, for any rationals $r_{0}$ and $r_{1}$ inside $\mathbf{I}$,

$$
\operatorname{cl}_{X} U_{r_{0}} \subseteq U_{r_{1}} \text { if and only if } r_{0}<r_{1} .
$$

Then, as demonstrated before the function $f: X \rightarrow \mathbf{I}$ defined by

$$
f(z)= \begin{cases}\inf \left\{r \in \mathbf{Q} \cap \mathbf{I}: z \in U_{r}\right\}, & \text { if } z \in U_{1} ; \text { or } \\ 1, & \text { if } z \notin U_{1}\end{cases}
$$

is continuous. As, for any $i \in\{0,1\}, f\left[F_{0}\right] \subseteq\{i\}$, the desired is proved. ${ }^{2}$

As a consequence of the lemma (3.3.4), every normal space is also a Tychonoff space.

For any cardinal $k, \mathbf{D}(\kappa)$ will denote a discrete space of cardinality $\kappa$. As such, any function whose domain is $\mathbf{D}(\kappa)$ and takes image in anu other topological space is continuous.

\footnotetext{
${ }^{2}$ The process of define such function $f$ by the means of the existence of a collection $\left\langle V_{r}\right\rangle_{r \in \mathbf{I} \cap \mathbf{Q}}$ satisfying \& is known as Urysohn onion shell argument. It is also used also to prove that any topological group that happens to be a $T_{1}$ space is a Tychonoff space. This property arise as a connection between the lattice of open subsets of $X$ and the ordered field of $\mathbf{R}$.
} 


\subsection{Convergence}

Let $X$ be a topological space and $\left\langle x_{n}\right\rangle$ a sequence of points within $X$; the usual definition of convergence says that $\left\langle x_{n}\right\rangle$ converges to a point $x \in X$ if and only if, for each neighbourhood $U$ of $x$ in $X$, one can find a $m \in \mathbf{N}$ such that $x_{n} \in U$ whenever $n \geq m$. Thus, it is required that a $\operatorname{large} e^{3}$ portion of such sequence to be within the neighbourhood $U$.

As said in the introduction, the notion of filter is one of the many notion that encapsulates the notion of largeness within a set. Indeed, if $\left\langle x_{n}\right\rangle$ is a sequence of points inscribed within a topological space $X$ and converging to a point $x \in X$, for any $U \in$ $v_{X}(x)$, let

$$
A_{U}=\left\{n \in \mathbf{N}: x_{n} \in U\right\} .
$$

The collection $\mathcal{F}=\left\{A_{U}: U \in \mathcal{V}_{X}(x)\right\}$ is a filter of $\wp(\mathbf{N})$ and represent the convergence structure of $V_{X}(x)$ inside $\wp(\mathbf{N})$. Moreover, the convergence of $\left\langle x_{n}\right\rangle$ can be restated as for each neighbourhood $V$ of $x$ in $X$, there is a $A \in \mathscr{F}$ with the property of $x_{n} \in V$, whenever $n \in A$. As the collection $\mathscr{F}$ has the FIP, one could restate the definition in terms of a ultrafilter that contains $\mathscr{F}$.

What is observed in the above paragraph, as a chance for abstraction, is that each ultrafilter of $\wp(\mathbf{N})$ can give birth to a notion of convergence. Indeed, if $S$ is a set and $P$ is an filter of $\wp(S)$, then a point $x$ of a topological space $X$ is said to be a $P$-limit of a collection $\left\langle x_{s}\right\rangle_{s \in S}$ inscribed in $X$ if and only if, for any neighbourhood $U$ of $x$ in $X$, one can find a $A \in P$ with the property $x_{s} \in U$ whenever $s \in A$. The set of all $P$-limits of $\left\langle x_{n}\right\rangle$ is denoted by $p-\lim _{s \in S} x_{n}$ or, by a slackness of the author, $p-\lim x_{s}$ when there is no danger of confusion. Thus, each ultrafilter of $\wp(S)$ codifies the convergence structure of $V_{X}(x)$; this will be useful when an algebraic structure is defined in the set of all ultrafilters of $\wp(\mathbf{N})$.

3.4.1 Theorem. Being $X$ a topological space, $S$ a set, $\left\langle x_{s}\right\rangle_{s \in S}$ a collection of $X$ 's elements and $P$ an filter of $\wp(S)$, the following proposition are true:

(3.4.1.1). If $A \in P$, then $P-\lim x_{s} \subseteq \operatorname{cl}_{X}\left\{x_{s}: s \in A\right\}$;

(3.4.1.2). if $P$ is a prime filter, then $\bigcap_{A \in \mathfrak{p}} \mathrm{cl}_{X}\left\{x_{s}: s \in A\right\}=\mathfrak{p}-\lim x_{s}$;

(3.4.1.3). if $X$ is Hausdorff, then either $P$ - $\lim x_{s}$ is empty or a singleton;

(3.4.1.4). if $Y$ is a topological space and $f: X \rightarrow Y$ is continuous, then $f\left[P-\lim x_{s}\right] \subseteq$ $P-\lim f\left(x_{s}\right)$; and

(3.4.1.5). $\operatorname{cl}_{X}\left\{x_{s}: s \in S\right\}=\bigcup_{P \in \operatorname{stn} \wp(S)} P-\lim x_{s}$.

Proof: To see the validity of (3.4.1.1), suppose the existence of a $P$-limit $x$ of $\left\langle x_{s}\right\rangle_{s \in S}$; then, for every neighborhood $U$ of $x$ in $X$, one can find a $B \in P$ such that

\footnotetext{
${ }^{3}$ In terms of cardinality, when compared with the whole $\mathbf{N}$
} 
$x_{s} \in U$, whenever $x \in B$. As $P$ is a non-trivial filter, $A \cap B \neq \emptyset$ and $A \cap B \subseteq B$. Thus,

$$
\emptyset \neq\left\{x_{s}: s \in A \cap B\right\} \subseteq U \cap\left\{x_{s}: s \in A\right\} .
$$

To prove (3.4.1.2), let $x$ be a point within the referred intersection; then, for every neighborhood $U$ of $x$ in $X$ and $A \in P$, the set $\left\{x_{s}: s \in A\right\}$ meets $U$ in concordance with the indexes inside some $A_{0} \subseteq A$. As $P$ is a prime filter, either $A_{0} \in P$ or $A_{1}=A \backslash A_{0}$ is a member of $P$. Hence, considering the case in which $A_{0}$ is not a member of $P$, the argument reaches an absurd for, in this particular case, $x$ would be a member of $\operatorname{cl}_{X}\left\{x_{s}: s \in A_{1}\right\}$.

If $x_{0}$ and $x_{1}$ are members of $P-\lim x_{s}$, as $P$ is a proper filter and is closed by intersection, there are no two open subsets of $X$ being each a neighborhood of $x_{0}$ and $x_{1}$ that could separates points of $\left\langle x_{s}\right\rangle_{s \in S}$ that are approaching $x_{0}$ or $x_{1}$ in the sense of $P$. Therefore, as $X$ is a Hausdorff space, $x_{0}$ must be equal to $x_{1}$, which settles (3.4.1.3).

If $y \in f\left[P-\lim x_{s}\right]$, then there is a $P$-limit $x$ of $\left\langle x_{s}\right\rangle_{s \in S}$ such that $f(x)=y$. As $f$ is continuous, for every neighborhood $V$ of $y$ in $Y$, one can find a neighborhood $U$ of $x$ in $X$ such that $f[U] \subseteq V$; as $x$ is a $P$-limit of $\left\langle x_{s}\right\rangle_{s \in S}$, one can find a $A \in P$ such that $\left\{x_{s}: s \in A\right\} \subseteq U$. Thus, $y$ is a $P$-limit of $\left\langle f\left(x_{s}\right)\right\rangle_{s \in S}$ for

$$
\left\{f\left(x_{s}\right): s \in A\right\} \subseteq f[U] \subseteq V .
$$

At last, (3.4.1.2) proves that

$$
\bigcup_{P \in \operatorname{stn} \wp(S)} P-\lim x_{s}=\bigcup_{P \in \operatorname{stn} \wp(S)} \bigcap_{A \in P} \operatorname{cl}_{X}\left\{x_{s}: s \in A\right\} \subseteq \operatorname{cl}_{X}\left\{x_{s}: s \in S\right\} .
$$

Conversely, given a $x \in \operatorname{cl}_{X}\left\{x_{s}: s \in S\right\}$, for every neighborhood $U$ of $x$ in $X$, there is a $A_{U} \subseteq S$ such that $\emptyset \neq\left\{x_{s}: s \in A_{U}\right\}=U \cap\left\{x_{s}: s \in S\right\}$. If $U_{0}$ and $U_{1}$ are neighborhood of $x$ in $X$, then $A_{U_{0} \cap U_{1}}=A_{U_{0}} \cap A_{U_{1}}$. Therefore, the colection of all $A_{U}$, as $U$ runs through the neighborhood of $x$ in $X$, can be extended to an ultrafilter $P$ of $\wp(S)$. Consequently, $x \in P-\lim x_{s}$.

For any point $x$ of a topological space $X$, let $\left\langle x_{s}\right\rangle$ be a collection of $X$ 's points and $P$ a filter of $S$ such that one can find a $x \in P-\lim x_{s}$. Then, the axiom of choice produces a function $\varphi: V_{X}(x) \rightarrow P$ such that, for any neighbourhood $U$ of $x$ in $X$, $V_{U}=\left\{x_{s}: s \in \varphi(U)\right\} \subseteq U$. Now, the collection $\left\{V_{U}: U \in V_{X}(x)\right\}$ has the FIP and thus generates an ultrafilter $u$ of $\wp(X)$. Obviously, $u$ contains $v_{X}(x)$.

If $\mathscr{F}$ is a filter of a topological space $X$, then a point $x \in X$ is said to be a limit of the filter $\mathscr{F}$ if such filter contains all neighbourhoods of $x$; the collection of all limits of a filter $\mathscr{F}$ of $X$ is denoted by $\lim \mathcal{F}$.

3.4.2 Lemma. A topological space $X$ is Hausdorff if and only if for every filter $\mathscr{F}$ of $X$ has at most one limit. 


\subsection{Subspaces}

Let $X$ be a set and $M$ be a subset of $X$. Then, the inclusion map $\iota_{A \rightarrow X}: M \rightarrow X$ is defined as $\iota_{M \rightarrow X}(x)=x$. The identity map $\iota_{X \rightarrow X}$ will be denoted as $\iota_{X}$.

Let $X$ be a topological space and $M$ a subset of $X$. Then, the topology of subspace $\tau_{M, X}$ for $M$ is the $\subseteq$-infimum of all topology for $M$ that makes $\iota_{M \rightarrow X}$ continuous. As, for any $A \subseteq X$,

$$
\iota_{M \rightarrow X}^{-1}[A]=A \cap M
$$

one can easily see that

$$
\tau_{M, X}=\left\{U \cap M: U \in \tau_{X}\right\} .
$$

The topological space $\left\langle M, \tau_{M, X}\right\rangle$ is said to be a subspace of the topological space $X$.

A predicate $\mathscr{P}$ concerning topological spaces is said to be hereditary if, for every topological space $X$ that has the property $\mathscr{P}$, all subspaces of $X$ also have the property $\mathscr{P}$. A property $\mathscr{P}$ is said to be hereditary with respect of the property $\mathcal{Q}$ if, for every topological space $X$ that has the property $\mathscr{P}$, all subspaces of $X$ having the property $\mathcal{Q}$ also have the property $\mathscr{P}$.

It is easy too see that being a $T_{1}$ space, a Hausdorff space, a regular space, or a Tychonoff space is hereditary. Clearly, using the Urysohn's Lemma (3.3.4), normality is hereditary with respect to closed subsets.

3.5.1 Lemma. Normality is hereditary with respect of $\mathrm{F}_{\sigma}$-sets.

Proof: Let $F$ be a $\mathrm{F}_{\sigma}$ set of $X$ and $\left\langle F_{n}\right\rangle$ a sequence of closed subsets of $X$ whose union is equal to $F$. Given any closed subset $G$ of $F$, for any $n \in \mathbf{N}$, the set $G_{n}=G \cap F_{n}$ is closed in $X$. Thus, if $V$ is a open set of $X$ that contains $G$, the normality of $X$ produces a open set $V_{n}$ of $X$ such that

$$
G_{n} \subseteq V_{n} \subseteq \mathrm{cl}_{X} V_{n} \subseteq V
$$

Thus, for any $n \in \mathbf{N}$

$$
G \subseteq \bigcup_{n \in \mathbf{N}}\left(F \cap V_{n}\right) \quad \text { and } \quad \operatorname{cl}_{F}\left(F \cap V_{n}\right) \subseteq F \cap V
$$

Evoquing the lemma (3.3.3), the proof is complete.

In general, normality is not hereditary as can be seen in [Eng89; LAS95].

A continuous injection $f$ from a topological space $X$ to a topological space $Y$ is said to be a homeomorphic embedding if there is a subspace $Y_{0}$ of $Y$ and a homeomorphism 
$f^{\prime}: X \rightarrow Y_{0}$ such that the following diagram commutes

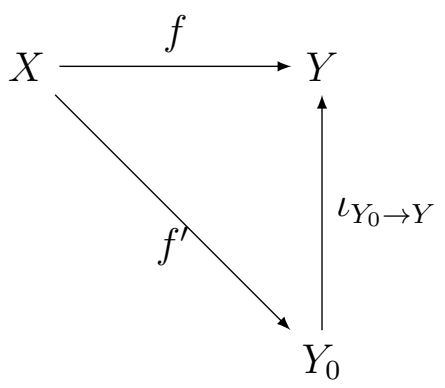

In the presence existence of a homeomorphic embedding from $X$ to $Y, X$ is said to be embeddable into $Y$.

\subsection{Product}

Let $\prod_{s \in S} X_{s}$ be the product of the collection of sets $\left\langle X_{s}\right\rangle_{s \in S}$. Then is possible to define the projection maps on the coordinate $s$ as the function $\mathrm{b}_{s}: \prod_{s \in S} X_{s} \rightarrow X_{s}$ given by $\mathrm{p}_{s}(x)=x(s)$.

The Tychonoff topology for the product $\prod_{s \in S} X_{s}$ of a collection of spaces $\left\langle X_{s}\right\rangle_{s \in S}$ is defined as the $\subseteq$-infimum of all topologies over the product that make every projection $\mathrm{p}_{s}$ continuous. Evidently $X$, together with a family $\left\langle h_{s}\right\rangle_{s \in S}$, is homeomorphic to the product of the family $\left\langle X_{s}\right\rangle_{s \in S}$ if and only if

prod.1 Given any space $Y$ and two continuous mappings $f, g: Y \rightarrow X$ such that, for every $s \in S, h_{s} \circ f=h_{s} \circ g$, then $f=g$; and

prod.2 given any space $Y$ that, for every $s \in S$, is linked to $X_{s}$ via a continuous function $f_{s}: Y \rightarrow X_{s}$ there is a continuous function $f: Y \rightarrow X$ such that $h_{s} \circ f=h_{s}$

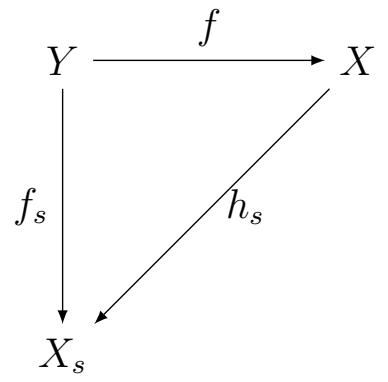

The continuous function $f$ in prod. 2 is called diagonal of the family $\left\langle f_{s}\right\rangle_{s \in S}$ and is denoted by $\triangle_{s \in S} f_{s}$. Evidently, in the canonical definition of the product, the diagonal has the definition

$$
\triangle_{s \in S} f_{s}(y)(s)=f_{s}(y) .
$$

The prod.1 assertion makes the diagonal unique. 
Thus, if $\sigma: S \rightarrow S$ is a permutation, then $\prod_{s \in S} X_{s}$ is homeomorphic to $\prod_{s \in S} X_{\sigma(s)}$. Indeed, using the characterization of products above, one can find continuous functions $f$ and $g$ such that

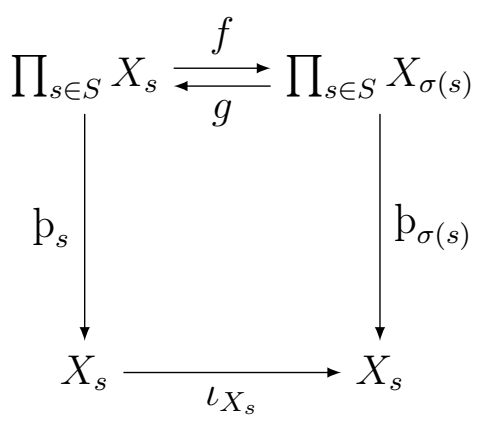

is a commutative diagram. As such, $f^{-1}=g$.

3.6.1 Lemma. Let $X$ be a topological space and $\left\langle Y_{s}\right\rangle_{s \in S}$ be a family of topological spaces. For every $s \in S$, suppose available a continuous function $f_{s}: X \rightarrow Y_{s}$. Then, the following are equivalent

SPC1 if $x \in X$ does not belongs to a closed subset $F$ of $X$, then there is a $s \in S$ such that $f_{s}(x) \notin \operatorname{cl}_{Y_{s}} f_{s}[F]$;

SPC2 $\mathscr{B}=\bigcup_{s \in S}\left\{f_{s}^{-1}[U]: U \in \tau_{Y_{s}}\right\}$ is a base of open subsets for $\tau_{X}$.

Proof: Assuming SPC1, let $U$ be an non empty open subset of $X$ and select a $x \in U$. Then, there is a $s \in S$ and a open set $V \in \tau_{Y_{s}}$ such that $f_{s}(x) \notin \operatorname{cl}_{Y_{s}} f_{s}[X \backslash U]$. Thus, is possible to find an open set $V$ of $Y_{s}$ such that $V$ contains $f_{s}(x)$ and $V$ does not meet $\mathrm{cl}_{Y_{s}} f_{s}[X \backslash U]$. Hence, using CF7,

$$
x \in f_{s}^{-1}[V] \subseteq f^{-1}\left[Y \backslash \operatorname{cl}_{Y_{s}} f^{-1}[X \backslash U]\right] \subseteq X \backslash U,
$$

which proves SPC2.

Now, if SPC2 holds, let $x \in X$ and $F$ be a closed subset of $X$ such that $x \notin F$. Then, there is a $s \in S$ and an open subset $V$ of $Y_{s}$ such that $x \in f_{s}^{-1}[V] \subseteq X \backslash F$. Thus, $f_{s}(s) \in V$ and $V \cap f_{s}[F]=\emptyset$, which proves that $f_{s}(x) \notin \operatorname{cl}_{Y_{s}} f_{s}[F]$.

A family of function $\left\langle f_{s}\right\rangle_{s \in S}$ as in (3.6.1) that satisfies SPC1 or, equivalently, SPC2, is said to separates points from closed subsets. With the same notations and hypotheses from (3.6.1), if $X$ is a $\mathrm{T}_{1}$ space, being $x$ and $y$ distinct points of $X$, one can find a $s \in S$ such that $f_{s}(x) \neq f_{s}(y)$; the collection $\left\langle f_{s}\right\rangle_{s \in S}$ separates points if, for any $x$ and $y$ distinct points of $X$, one can find a $s \in S$ such that $f_{s}(x) \neq f_{s}(y)$. The proof of the following result can be found in [Eng89]:

3.6.2 Theorem. (The Diagonal Theorem) Let $X$ be a topological space and $\left\langle Y_{s}\right\rangle_{s \in S}$ be a collection of topological spaces such that, for every $s \in S, Y_{s}$ is linked to $X$ via a continuous map $f_{s}: X \rightarrow Y_{s}$. If the family $\left\langle f_{s}\right\rangle_{s \in S}$ separates points, then the diagonal mapping $\triangle_{s \in S} f_{s}$ is injective. Furthermore, if $\left\langle f_{s}\right\rangle_{s \in S}$ also separates points from closed subsets, then $\triangle_{s \in S} f_{s}$ is a homeomorphic embedding. 


\subsection{Compactness}

A cover by open subsets for a topological space $X$ is a collection $\mathcal{A}$ of open subsets of $X$ such that $X=\bigcup \mathcal{A}$. A Hausdorff space $X$ for which every cover by open $\mathcal{A}$ subsets contains a finite cover (said sub-cover) for $X$ is said to be a Compact space ${ }^{4}$.

The finiteness of the cover that must exist to ensure compactness has a deep connection with the theory of filters, namely by the use of complementation and closed subsets. Indeed, if $\mathcal{A}$ is a cover by open subsets for a space $X$, then the collection $\mathcal{F}$ of all complementation of members of $\mathcal{A}$ is a collection of closed subsets of $X$ that has empty intersection. Moreover, by the definition of compactness, if $X$ is compact, then any finite sub-collection of $\mathscr{F}$ must have empty intersection. Thus, the following result is proved:

3.7.1 Lemma. A Hausdorff space is compact if and only if any collection of closed subsets of $X$ that has the FIP has non empty intersection.

3.7.2 Corolary. If $X$ is a quasi-compact space and $\left\langle x_{s}\right\rangle$ is a collection of elements of $X$, for any prime filter $p$ of $S, p-\lim x_{s}$ is not empty. Moreover, if $X$ is compact, then $p-\lim x_{s}$ is a singleton.

Proof: As observed in (3.4.1.2),

$$
\bigcap_{A \in p} \operatorname{cl}_{X}\left\{x_{s}: s \in A\right\}=p-\lim x_{s}
$$

whenever $p$ is prime; as $p$ is filter, the collection $\left\{\left\{x_{s}: s \in A\right\}: A \in p\right\}$ must fulfills the FIP, which proves that $p-\lim x_{s}$ is not empty. Therefore, if $X$ is compact, its property of being Hausdorff ensures, as observed in (3.4.1.3), that $p$ - $\lim x_{s}$ is constituted at most of one element.

For any space $X$, every family $\mathscr{F}$ composed by closed subsets of $X$ that has the FIP extends to an ultrafilter $p$ of $X$. Consequently, if $X$ is a quasi-compact space, then $\bigcap p$ is not empty; moreover, as $p$ is an ultrafilter, it must contain every neighbourhood of any element within its intersection and thus converges to every point present in its intersection. Reciprocally, if every ultrafilter of $X$ converges, then $X$ must be compact; indeed, if $\mathscr{F}$ is a collection of closed subsets of $X$ having the FIP, then it can be extended to an ultrafilter $p$ that converges to a point $x \in \bigcap P$. But, as $\bigcap p \subseteq \bigcap \mathcal{F}$, one has the following:

3.7.3 Lemma. A space $X$ is quasi-compact if and only if every ultrafilter of $X$ converges. Moreover, a space $X$ is compact if and only if every ultrafilter converges to an unique point.

\footnotetext{
${ }^{4}$ The reader should be aware that the definition of compact space given here is not the usual, which requires only property that every cover by open subsets must contains a finite cover by open subsets. A space that only fulfills this property will be called Quasi-compact space
} 
The reader should be now aware of the similarities between the lemma (3.7.3) and the Cauchy properties of the real line (i.e. every Cauchy sequence converges), which is behind all mechanism of constructing the Čech-Stone compactification, as the Cauchy properties of sequences is behind the completion of a metric space.

Let $X$ and $Y$ be topological spaces and $f: X \rightarrow Y$ a continuous function. For any compact set $K \subseteq X$, the image of $K$ under $f$ must be a compact set of $Y$. Thus, as any closed set within a compact space is compact; moreover, if $X$ is compact, then $f$ must be closed (i.e. it maps closed subsets to closed subsets).

3.7.4 Lemma. Any continuous bijection from a compact space $X$ to a space $Y$ is a homeomorphism.

\subsection{The Stone's Duality}

Let $L$ be a bounded distributive lattice and denote by $\operatorname{stn}(L)$ the collection of all ultrafilters of $L$, then is possible to define a map $d: L \rightarrow \wp(\operatorname{stn}(L))$ by

$$
d(x)=\{p \in \operatorname{stn}(L): x \in p\}
$$

called the Stone's duality of the lattice $L$. It is clear that $d(\mathbb{O})=\emptyset$ and $d(\mathbb{1})=\operatorname{stn} L$.

3.8.1 Lemma. For any distributive bounded lattice, its Stone's duality is a bounded monomorphism (i.e. $d$ is a injective homorphism that maps the minimum of $L$ to $\emptyset$ and the maximum to $\operatorname{stn} L)$.

Proof: Given two elements $x$ and $y$ of $L$ such that $x \curlywedge y$ is a member of an $L$ 's ultrafilter $P$, as $x \curlywedge y \preceq x$ and $x \curlywedge y \preceq y$ both $x$ and $y$ are member of $P$, thus

$$
\begin{gathered}
d(x \curlywedge y)=\{p \in \operatorname{stn}(L): x \curlywedge y \in p\}=\{p \in \operatorname{stn}(L): x \in p \wedge y \in p\}= \\
=\{p \in \operatorname{stn}(L): x \in p\} \cap\{p \in \operatorname{stn}(L): y \in p\}=d(x) \cap d(y) .
\end{gathered}
$$

Recall that any ultrafilter in a distributive lattice is a prime filter, thus

$$
\begin{gathered}
d(x \curlyvee y)=\{p \in \operatorname{stn}(L): x \curlyvee y \in p\}=\{p \in \operatorname{stn}(L): x \in p \vee y \in p\}= \\
\quad=\{p \in \operatorname{stn}(L): x \in p\} \cup\{p \in \operatorname{stn}(L): y \in p\}=d(x) \cup d(y) .
\end{gathered}
$$

Now, if $d(x)=d(y)$, then $y$ is a member of $\mathbb{E}(x)$, the filter generated by $\{x\}$. Thus $x \leq y$; analogously, one can obtain $y \leq x$.

3.8.2 Theorem. [Sto35] If $L$ is a bounded distributive lattice, then there exists a topology $\tau_{S}$ on $\operatorname{stn}(L)$ such that $\mathcal{Q}[L]$ is a base of closed for $\left\langle\operatorname{stn}(L), \tau_{S}\right\rangle$. Also, $\left\langle\operatorname{stn}(L), \tau_{S}\right\rangle$ is a quasi-compact space. 
Proof: As $d$ is a bounded morphism and $\bigcap \mathbb{Q}[L]=\emptyset$, the colection $d[L]$ is a base for closed sets for some topology $\tau_{S}$ of $\operatorname{stn}(L)$. Now, let $A$ be a subset of $L$ having the FIP; then, there is an ultrafilter $p \in \operatorname{stn}(L)$ such that $A \subseteq p$, i.e., for every $x \in A, p \in d(x)$. Thus, $p \in \bigcap_{x \in A} d(x)$, which proves that $\left\langle\operatorname{stn}(L), \tau_{S}\right\rangle$ is a quasi-compact space.

3.8.3 Lemma. For a distributive bounded lattice $L, \operatorname{stn}(L)$ is Hausdorff if and only if given two disjoint elements $a$ and $b$ of $L$, one can find elements $x$ and $y$ of $L$ such that $a \curlywedge x=b \curlywedge y=\mathbb{0}$ and $x \curlyvee y=\mathbb{1}$.

Proof: Suppose that $\operatorname{stn}(L)$ is Hausdorff, then $\operatorname{stn}(L)$ is a normal space, for the quasi-compactness of $\operatorname{stn}(L)$. Thus, for any disjoint elements $a$ and $b$ of $L$, one can find elements $x$ and $y$ such that $d(a) \subseteq \operatorname{stn}(L) \backslash d(x), d(b) \subseteq \operatorname{stn}(L) \backslash d(y)$ and

$$
(\operatorname{stn}(L) \backslash \mathcal{Q}(x)) \cap(\operatorname{stn}(L) \backslash \mathcal{Q}(y))=\emptyset
$$

Hence, $a \curlywedge x=b \curlywedge y=\mathbb{O}$ and $x \curlyvee y=\mathbb{1}$.

Conversely, let $p$ and $q$ be two distinct ultrafilters of $L$. If, without loss of generality, $a$ is an element of $p$ that is not listed among the elements of $q$; then, using 2.3.5, one can find a element $b \in q$ such that $a \curlywedge b=\mathbb{0}$; thus, by the hypothesis of $L$, is possible to find elements $x$ and $y$ of $L$ such that $a \curlywedge x=b \curlywedge y=\mathbb{O}$ and $x \curlyvee y=\mathbb{1}$. Thus, $p \in \operatorname{stn}(L) \backslash d(x)$, $q \in \operatorname{stn}(L) \backslash d(y)$ and

$$
(\operatorname{stn}(L) \backslash d(x)) \cap(\operatorname{stn}(L) \backslash d(y))=\emptyset,
$$

which proves that $\operatorname{stn}(L)$ is Hausdorff.

Any distributive bounded lattice $L$ that fulfills any of the equivalent conditions of the lemma 3.8.3 is said to be normal ${ }^{5}$. Evidently, for any set $X, \wp(X)$ is a normal lattice. Unfortunately, the lattice from the example 2.5.2 is an example of a distributive, bounded from above and not normal lattice.

3.8.4 Lemma. Let $R$ and $L$ be normal lattices and $f: R \rightarrow L$ be a bounded lattice morphism. Then, there is a continuous function $\varphi: \operatorname{stn}(L) \rightarrow \operatorname{stn}(R) \operatorname{such}$ that, for every $x \in R, \varphi^{-1}\left[d_{R}(x)\right]=d_{L}(f(x))$.

Proof: As the dualities $d_{L}$ and $d_{R}$ are isomorphisms between its images $\mathcal{L}$ and $\mathcal{R}$, respectively, one can define $g: \mathscr{R} \rightarrow \mathcal{L}$ by $g(F)=d_{L} \circ f \circ d_{R}^{-1}(F)$. By the definition, $g$ is a morfism and

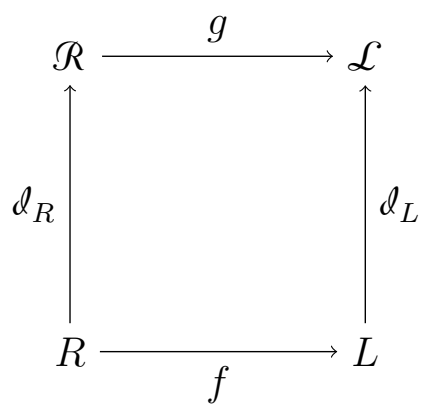

\footnotetext{
${ }^{5}$ The reader is hereby invited to compare the definitions of a normal lattice to the definition of a normal base of open subsets for a $\mathrm{T}_{1}$ space given by (NBO1) and (NBO2)
} 
is a commutative diagram. For any $p \in \operatorname{stn}(L)$, let $\mathscr{F}_{p}$ be the family of all closed sets belonging to $\mathcal{L}$ that has $p$ among its elements. Then, the family

$$
\mathscr{L}_{p}=\left\{G \in \mathscr{R}: g(G) \in \mathscr{F}_{p}\right\}
$$

has the FIP. As $\operatorname{stn}(R)$ is a compact space, $\bigcap \mathscr{L}_{p}$ is not empty. Now, if there are distinct ultrafilters $q_{0}$ and $q_{1}$ inside $\bigcap \mathcal{G}_{p}$, then one can find elements $x$ and $y$ of $R$ such that $x \notin q_{0}$, $y \notin q_{1}$ and $x \curlyvee y=\mathbb{1}_{R}$. Thus, without loss of generality, as $d_{L}(f(x)) \cup d_{L}(f(y))=\mathcal{L}$, $p \in d_{L}(f(x))$. As $g \circ d_{R}=f \circ d_{L}, d_{R}(x) \in \mathcal{L}_{p}$, which is absurd. Thus, one can define $\varphi: \operatorname{stn}(L) \rightarrow \operatorname{stn}(R)$ by setting $\varphi(p)$ to be the unique member of $\bigcap \mathcal{L}_{p}$.

Moreover, given any ultrafilter $p$ of $L$ and $x \in R, \varphi(p) \in d_{R}(x)$ if and only if $p \in g\left(d_{R}(x)\right)$, which proves the continuity of $\varphi$.

3.8.5 Lemma. The Stone space of a Boolean algebra has a base of open-and-closed subsets (i.e it is zero-dimensional).

Proof: Let $B$ be a Boolean algebra and $d: B \rightarrow \operatorname{stn}(B)$ be the Stone duality of $B$. Then, the colection

$$
\mathscr{B}=\{d(x): x \in B\}=\{\{q \in \operatorname{stn}(B): x \in q\}: x \in B\}
$$

is a base of closed sets for $\operatorname{stn}(B)$. As the duality is a monomorphism between $B$ and $\wp(\operatorname{stn}(B))$, every set in the form $d(x)$ is also open, for $d(x)=\operatorname{stn}(B) \backslash d\left(x^{*}\right)$.

\subsection{Topological translation of order properties}

3.9.1 Lemma. [Hin88] Let $\varphi$ be predicate discoursing about elements of some Boolean algebra $B$ and

$$
\mathscr{R}=\{p \in \operatorname{stn} B: \forall a \in p(\varphi(a))\} .
$$

Then, the following assertions are equivalent:

(3.9.1.1). For every $a \in B, \varphi(a) \rightarrow(d(a) \cap \mathscr{R} \neq \emptyset)$; and

(i) $\neg \varphi(\mathbb{D})$;

(ii) for any elements $a$ and $b$ of $B$ such that $a \leq b, \varphi(a) \rightarrow \varphi(b)$;

(iii) for any elements $a$ and $b$ of $B, \varphi(a \curlyvee b) \rightarrow \varphi(a) \vee \varphi(b)$.

Proof: Assuming (3.9.1.1), it is obivious that (i) occurs because $d(\mathbb{O})=\emptyset$. Now, if $a$ and $b$ are elements of $B$ such that $a \leq b$ and $\varphi(a)$, then is possible to find a $p \in \mathcal{Q}(a) \cap \mathscr{R}$. As $p$ is a filter and $a \leq b, b \in p$; thus $p \in \mathcal{d}(b)$. Finally, if $p \in \mathcal{d}(a \curlyvee b) \cap \mathcal{R}$, then $a \curlyvee b \in p$; as $p$ is a prime filter, $a \in p$ or $b \in p$, which translates to $\varphi(a)$ or $\varphi(b)$.

Conversely, define $\mathscr{F}$ to be the collection of all proper filters of $B$ whose elements make $\varphi$ true by evaluation. $\mathcal{F}$ is not empty since the vality of (i), (ii) and (iii) implies 
$\varphi(\mathbb{1})$. As the union of a $\subseteq$-linear family of proper filters of $B$ is a proper filter of $B$, the Kuratowski-Zorn lemma produces a $\subseteq$-maximal element $p$ of $\mathscr{F}$. If $p$ is not an ultrafilter of $B$, one can find a $b \in B$ such that neither $b$ nor $b^{*}$ are listed among the elements of $p$. Thus,

$$
F_{0}=\{a \in B: \exists c(c \in p \wedge c \curlywedge b \leq a)\}
$$

and

$$
F_{1}=\left\{a \in B: \exists c\left(c \in p \wedge c \curlywedge b^{*} \leq a\right)\right\}
$$

are proper filters of $B$. Indeed, let $a \in F_{0}$ and $d \in B$ such that $a \leq d$; then, one can find a $c \in p$ such that $b \curlywedge c \leq a \leq d$, and thus $d \in F_{0}$. If $a_{0}, a_{1} \in F_{0}$, then the definition of this set produces, for each $i \in\{0,1\}$, a $c_{i} \in p$ such that $b \curlywedge c_{i} \leq a_{i}$; hence

$$
b \curlywedge\left(c_{0} \curlyvee c_{1}\right)=\left(b \curlywedge c_{0}\right) \curlyvee\left(b \curlywedge c_{1}\right) \leq a_{0} \curlyvee a_{1} \text {. }
$$

For last, if $\mathbb{O} \in F_{0}$, then there is a $c \in p$ such that $b \curlywedge c=\mathbb{0}$; consenquently, $c \leq b^{*}$, which is absurd.

Moreover, for any $a \in p$, one can prove that

$$
a \curlywedge b \leq a \text { and } a \curlywedge b^{*} \leq a .
$$

Hence, $F_{0}$ and $F_{1}$ circumscribe of $p$, which proves that neither $F_{0}$ nor $F_{1}$ are listed among the elements of $\mathcal{F}$. By the definition of $\mathcal{F}$, one can select some $c$ and $d$ among the elements of $p$ such that

$$
\neg \varphi(b \curlywedge c) \text { and } \neg \varphi\left(b^{*} \curlywedge d\right)
$$

As $p$ is a filter, $\varphi(c \curlywedge d)$ and thus, by (iii), either $b \curlywedge c \curlywedge d$ or $b^{*} \curlywedge c \curlywedge d$ must validate $\varphi$ by evaluation. Consequently, by (ii), either $\varphi(c \curlywedge b)$ or $\varphi\left(b^{*} \curlywedge d\right)$, which is absurd.

3.9.2 Corolary. In (3.9.1), the set $\mathscr{R}$ is a closed subset of $\operatorname{stn} B$.

3.9.3 Corolary. Let $\varphi$ and $\psi$ be predicates discoursing about elements of some Boolean algebra $B$ such that $\varphi$ satisfies one of the equivalent conditions of (3.9.1). If $\mathscr{R}$ is as defined in (3.9.1) for some Boolean algebra $B$, let

$$
\mathcal{S}=\{p \in \operatorname{stn} B: \forall a \in p(\psi(a))\} .
$$

Then, the following are equivalent:

(3.9.3.1). $\mathscr{R} \subseteq \mathcal{S}$; and

(3.9.3.2). for all $a \in B, \varphi(a) \rightarrow \psi(a)$. 


\section{CHAPTER 4}

\section{Compactifications}

\subsection{Extension of continuous functions}

Let $M$ be a subspace of a topological space $X$. A continuous function $f$ defined in $M$ and assuming its values in a topological space $Y$ is said to be continuous extendable over $X$ if there is a continuous function $F: X \rightarrow Y$ such that $F \uparrow_{M}=f$; evidently, this definition is equivalent to ask for the existence of a continuous function $F: X \rightarrow Y$ such that

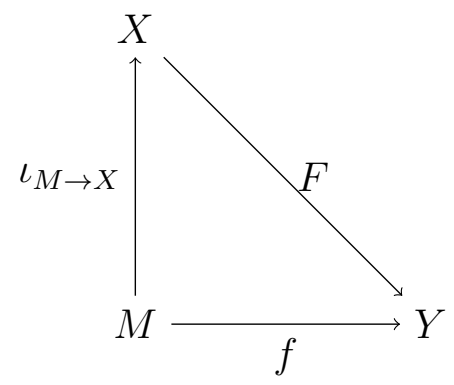

is a commutative diagram.

4.1.1 Theorem. [Ury25] Any continuous function defined in a subspace $M$ of a topological space $X$ and assuming its values in $\mathbf{I}$ is continuously extendable over $X$ if and only if any two disjoint functionally closed subsets of $M$ are completely separated in $X$.

Proof: Let $F$ and $G$ be any disjoint and functionally closed subsets of $M$. Then, there is a continuous function $f: M \rightarrow \mathbf{I}$ such that $F=f^{-1}[\{0\}]$ and $G=f^{-1}[\{1\}]$. Thus, if $f$ is extendable over $X$ by a continuous function $F: X \rightarrow \mathbf{I}, F$ and $G$ are completely separated by $F$.

Conversely, proceeding inductively, suppose available a continuous function $f_{n}$ : 
$M \rightarrow \mathbf{I}$ such that $\left|f_{n}\right| \leq 2^{-n}$. By defining the sets

$$
F_{n}=\left\{x \in M: f_{1}(x) \leq \frac{1}{3}\right\} \quad \text { and } \quad G_{n}=\left\{x \in M: f_{1}(x) \geq \frac{2}{3}\right\}
$$

the hypothesis that both $F_{n}$ and $G_{n}$ are completely separated in $X$ produces a continuous function $g_{n}: X \rightarrow \mathbf{I}$ that, without loss of generality, can be assumed to have norm not exceeding $2^{-n}$ and complete separates the sets $F_{n}$ and $G_{n}$. Then, by setting $f_{n+1}=$ $4^{-1}\left(f_{n}-g_{n} \uparrow_{M}\right)$, one have $\left|f_{n+1}\right| \leq 2^{-n-1}$.

Starting with any continuous function $f_{0}: M \rightarrow \mathbf{I}$, the above induction produces two sequences of continuous functions, namely $\left\langle f_{n}\right\rangle$ and $\left\langle g_{n}\right\rangle$ such that, for all natural $n$, the values of $f_{n}$ and $g_{n}$ does not exceed $2^{-n}$. Thus, the Weierstrass's M-test tesfies the existence and the continuity of the function $g$ defined by the uniformly and absolutely convergent series $\sum_{n \geq 0} g_{n}$.

Now, given a natural number $n$,

$$
\left(g_{0}+g_{1}+\cdots+g_{n}\right) \uparrow_{M}=\left(f_{0}-f_{1}\right)+\left(f_{1}-f_{2}\right)+\cdots+\left(f_{n}-f_{n+1}\right)=f_{1}-f_{n+1} .
$$

As $f_{n+1} \rightarrow 0, g \uparrow_{M}=f_{0}$. Thus, $g$ is a continuous extension of $f_{0}$ over $X$.

A subspace $M$ of a topological space $X$ is said to be $C^{*}$-embeddable if every bounded real function defined in $M$ is continuous extendable over $X$. In the presence of Urysohn's Lemma (3.3.4) and the theorem (4.1.1) one can easily prove that

4.1.2 Corolary. [Ury25] Every closed subspace of a normal space is $\mathrm{C}^{*}$-embeddable.

Let $X$ be a topological space and $D$ a dense subset of $X$. Then, one defines

$$
\operatorname{ex}_{X, D}(A)=X \backslash \operatorname{cl}_{X}(D \backslash A)
$$

Given any $A_{0}, A_{1} \subseteq X$,

$$
\begin{aligned}
\operatorname{ex}\left(A_{0}\right) \cap \operatorname{ex}\left(A_{1}\right) & =\left[X \backslash \operatorname{cl}_{X}\left(D \backslash A_{0}\right)\right] \cap\left[X \backslash \operatorname{cl}_{X}\left(D \backslash A_{1}\right)\right]= \\
& =X \backslash\left(\operatorname{cl}_{X}\left(D \backslash A_{0}\right) \cup \operatorname{cl}_{X}\left(D \backslash A_{1}\right)\right)=X \backslash\left(\operatorname{cl}_{X}\left(D \backslash\left(A_{0} \cap A_{1}\right)\right)\right)= \\
& =\operatorname{ex}_{X, D}\left(A_{0} \cap A_{1}\right) .
\end{aligned}
$$

Moreover, it is easy to see that, for each open set $U$ of $X$,

$$
U=\operatorname{ex}_{X, D} U
$$

because $U=X \backslash \operatorname{cl}_{X}(X \backslash U)$.

4.1.3 Lemma. Let $X$ be a regular space and $V$ a family of open subsets of $X$. If

$$
\mathcal{T}=\left\{W \in \tau_{X}: \exists V \in \mathcal{V}(W \subseteq V)\right\}
$$

then $\bigcup \mathcal{V}=\bigcup \mathcal{T}$. 
4.1.4 Theorem. [Sun89; PW88] Concerning a continuous map $f$ defined on a dense subspace $M$ of a topological space $X$ and assuming its values on a regular space $Y$, the following are equivalent:

(4.1.4.1). $f$ is continuous extendable over $X$;

(4.1.4.2). given any point $x \in X$,

$$
\mathscr{F}_{x}=\left\{A \subseteq Y: \exists U\left(U \in \mathcal{V}_{x} \wedge f[U \cap A] \subseteq M\right)\right\}
$$

is a convergent filter on $Y$;

(4.1.4.3). given an open cover $\mathcal{A}$ for $Y$, there is an open cover $\mathscr{B}$ for $X$ such that $\{U \cap M: U \in \mathscr{B}\}$ refines the open cover $\left\{f^{-1}[V]: V \in \mathcal{A}\right\}$; and

(4.1.4.4). if $\mathcal{A}$ is an open cover for $Y$, then

$$
\left.\left\{\operatorname{ex}_{X, M} f^{-1}[V]\right): V \in \mathcal{A}\right\}
$$

is an open cover for $X$.

Proof: (4.1.4.1) $\rightarrow($ 4.1.4.2). Let $F: X \rightarrow Y$ be an extension of $f$; then, for every neighbourhood $\mathrm{V}$ of $F(x)$ there is a neighbourhood $U$ of $x$ such that $F[U] \subseteq V$; thus, $V \in \mathcal{F}_{x}$.

(4.1.4.2) $\rightarrow(4.1 .4 .3)$. For every $x \in X$, let $F(x)$ be the limit of $\mathscr{F}_{x}$. Then, choosing a $V \in \mathcal{A}$ such that $F(x) \in V$ there is a neighbourhood $U_{x}$ of $x$ such that $f\left[U_{x} \cap A\right] \subseteq V$. Evidently, $\mathbb{B}=\left\{U_{x}: x \in X\right\}$ is a open cover for $X$.

The implication $(4.1 .4 .3) \rightarrow(4.1 .4 .4)$ is imediate.

$(4.1 .4 .4) \rightarrow(4.1 .4 .1)$ One can define the function $g^{*}: \tau_{Y} \rightarrow \tau_{X}$ as

$$
g^{*}(V)=\bigcup\left\{\operatorname{ex}_{X, M} f^{-1}[W]: W \in \mathscr{R}_{V}\right\}
$$

As the operator $\operatorname{ex}_{X, M}$ preserves finite intersections, so does $g^{*}$. Moreover, $g^{*}$ preserves the containment relation. Now, if $V$ is a family of open subsets of $Y$, the regularity of $Y$ implies that $\bigcup \mathcal{V}=\bigcup \mathcal{T}$, where

$$
\mathcal{T}=\left\{W \in \tau_{X}: \exists V \in \mathcal{V}(W \subseteq V)\right\} .
$$

Additionally, if $W \in \mathcal{T}$ and $V \in \mathcal{V}$ are so that $\operatorname{cl}_{Y} W \subseteq V$, then $\operatorname{ex}_{X, M} f^{-1}[W] \subseteq g^{*}(V)$. Thus, if $V$ is an open subset of $Y$ such that $\operatorname{cl}_{Y} V \subseteq \cup \mathcal{V}$, then $\mathcal{T} \cup\left\{Y \backslash \operatorname{cl}_{Y} V\right\}$ is a cover by open subsets for $Y$. Then the hypothesis testifies that

$$
\left\{\operatorname{ex}_{X, M} f^{-1}[W]: W \in \mathcal{T}\right\} \cup\left\{\operatorname{ex}_{X, M} f^{-1}\left[Y \backslash \operatorname{cl}_{Y}(V)\right]\right\}
$$

is a cover by open subsets for $X$ and, evidently, as $\operatorname{ex}_{X, M} f^{-1}[V] \cap \operatorname{ex}_{X, M} f^{-1}\left[Y \backslash \operatorname{cl}_{Y}(V)\right]=$ $\emptyset$,

$$
\operatorname{ex}_{X, M} f^{-1}[V] \subseteq \bigcup\left\{\operatorname{ex}_{X, M} f^{-1}[W]: W \in \mathcal{T}\right\} \subseteq \bigcup_{U \in \mathcal{V}} g^{*}(U)
$$


Therefore,

$$
g^{*}(\bigcup v)=\bigcup\left\{\operatorname{ex}_{X, M} f^{-1}[V]: V \in \mathscr{R}_{\cup} v\right\} \subseteq \bigcup_{U \in \mathcal{V}} g^{*}(U)
$$

Because $g^{*}$ preserves the containment, it is easy to see that $g^{*}$ preserves arbitrary unions. Moreover, as $Y$ is a regular space, for each open set $V$ of $Y$ the colection $\mathscr{R}_{V}$ of all open sets $W$ of $Y$ such that $\operatorname{cl}_{Y} W \subseteq V$ is not empty.

Now, let $g_{*}: \tau_{X} \rightarrow \tau_{Y}$ be given by

$$
g_{*}(U)=\bigcup\left\{V \in \tau_{Y}: U \subseteq g^{*}(U)\right\}
$$

Then, given an open set $V$ of $Y$ and an open set $U$ of $X$ such that $V \subseteq g_{*}(U)$, then

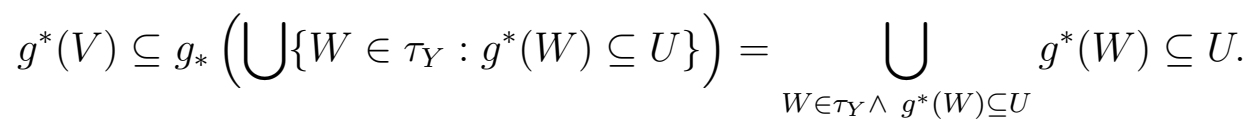

Conversely, if $g^{*}(V) \subseteq U$, by definition, $V \subseteq g_{*}(U)$.

By definition, for each $x \in X$ there is a closed set $F_{x}$ of $Y$ such that $g_{*}(X)$ $\left.\operatorname{cl}_{X}(\{x\})\right)=Y \backslash F$. The cases $F_{x}=\emptyset$ cannot be true, for otherwise it would imply that $g^{*}(Y)=X \backslash \operatorname{cl}_{X}(\{x\})$ and contradict the fact that $g^{*}(Y)=Y$. If is the case that $F_{x}$ has two distinct points, say $y_{0}$ and $y_{1}$, one can find two open sets $V_{0}$ and $V_{1}$ of $Y$ that disjoint separate these two points; in this way, it would result a $i \in\{0,1\}$ that testifyies $g^{*}\left(V_{i}\right) \subseteq X \backslash \operatorname{cl}_{X}\{x\}$ and therefore $V_{i} \subseteq Y \backslash F$, which is absurd for $y_{i} \in V_{i} \cap F$. Thus, $F_{x}$ consists of a single point. Thus, one can define a map $g: X \rightarrow Y$ such that, for each point $x$ of $X, g(x)$ is the unique point inside $F_{x}$.

For each $x \in X$ and any open set $V$ of $Y$,

$$
\begin{aligned}
g^{-1}[V] & =\{x \in X: g(x) \in V\}=\left\{x \in X: V \nsubseteq Y \backslash F_{x}\right\}= \\
& =\left\{x \in X: V \nsubseteq g_{*}\left(X \backslash \operatorname{cl}_{X}\{x\}\right)\right\}=\left\{x \in X: g^{*}(V) \nsubseteq X \backslash \operatorname{cl}_{X}\{x\}\right\}= \\
& =\left\{x \in X: g^{*}(V) \cap \operatorname{cl}_{X}\{x\} \neq \emptyset\right\}=\left\{x \in X: x \in g^{*}(V)\right\}=g^{*}(V),
\end{aligned}
$$

proving that $g$ is continuous.

Now note that, for each open set $V$ of $Y$,

$$
g^{*}(V) \cap M=\bigcup\left\{\left(\operatorname{ex}_{X, M} f^{-1}[W]\right) \cap M: W \in \mathcal{V}_{V}\right\}=\bigcup\left\{f^{-1}[W]: W \in \mathcal{V} \cdot V\right\}=f^{-1}[V]
$$

Thus, $g^{-1}[V] \cap M=f^{-1}[V]$.

Finally, if there is a point $x \in M$ such that $f(x) \neq g(x)$, then one can find an open set $V$ of $Y$ such that $f(x) \in V$ and $g(x) \notin V$. Since $x \in f^{-1}[V]=g^{-1}[V] \cap M$, this claim is absurd. Thus, $g$ is a continuous extension of $f$ over $X$.

4.1.5 Corolary. [Eng89; Tă52] A continuous map $f$, defined on a dense subspace $M$ of a topological space $X$ and assuming its values on a compact space $Y$, is continuous extendable over $X$ if and only if for every pair $F_{0}$ and $F_{1}$ of disjoint closed subsets inscribed in $Y$ the inverse images $f^{-1}\left[F_{0}\right]$ and $f^{-1}\left[F_{1}\right]$ have disjoint closures in $X$. 
Let $X$ and $Y$ be topological spaces and $A$ a subspace of $X$. Given a continuous map $f: A \rightarrow Y$ and $x \in \operatorname{cl}_{X} A$, a point $y \in Y$ is said to be a limit of $f$ on the point $x$ if, for every neighbourhood $V$ of $y$, there is a neighbourhood $U$ of $x$ such that $f[A \cap U] \subseteq V$. Denote by $\lim _{a \rightarrow x} f(a)$ the limit $y$, when there is any.

4.1.6 Corolary. Let $A$ be a subspace of $X$ and $f: A \rightarrow Y$ a continuous map of $A$ to a regular space $Y$. Then, $f$ extends to $\operatorname{cl}_{X} A$ if and only if, for every $x \in \operatorname{cl}_{X} A$, exists $\lim _{a \rightarrow x} f(a)$

\subsection{Compactifications}

Let $X$ be a topological space, then the weight of $X$ is defined as

$$
\mathrm{w}(X)=\min |\mathscr{B}|: \mathscr{B} \text { is a base of open subsets for } X .
$$

It's easy to see that if $Y$ and $X$ are homeomorphic spaces, then they have the same weight. As is done in [Eng89], one can prove that, for any cardinal $\kappa$ and any space $X$ whose weight does not exceed $\kappa$, given a base $\mathscr{B}$ for $X$, one can find a base $\mathcal{B}_{0} \subseteq \mathscr{B}$ satisfying $\left|\mathcal{B}_{0}\right| \leq \kappa$.

If $X$ is a Tychonoff space whose weight is $\kappa$, then there is a family $\mathcal{B}$ of functionally open sets of $X$ that is a base for $X$ and has cardinality $\kappa$. Thus, for every $U \in \mathscr{B}$, there is a continuous function $f_{U}: X \rightarrow \mathbf{I}$ such that $U=f^{-1}[[0,1[]$. Moreover, as $X$ is a Tychonoff space, and consequently a $\mathrm{T}_{0}$ space, the family $\left\{f_{U}: U \in \mathscr{B}\right\}$ separates points and separates points from closed sets. Therefore, the diagonal theorem 3.6.2 witnesses that the diagonal map $\triangle_{U \in \mathcal{B}} f_{U}$ is a homeomorphic embedding of $X$ in $\mathbf{I}^{\kappa}$. Thus, the Tychonoff separation axiom is equivalent to be homeomorphic to a dense subset of a compact space.

A compactification of a Tychonoff space is a pair $\langle c X, x\rangle$ in which $c X$ is a compact space and $c: X \rightarrow c X$ is a homeomorphic embedding that maps $X$ as a dense subspace of $c X$. Clearly, as seen above, a space $X$ has a compactification if and only if is a Tychonoff space. For conveniance, a compactification $\langle c X, x\rangle$ of a Tychonoff space $X$ will be denoted by $c X$, where the homeomorphic embedding $c$ will be understood from the notation.

To distinguish compactification by homeomorphic maps, is possible to define an order in the transversal set of all compactification of $X$, namely $\mathscr{K}(X)$ : given two compactifications $c_{0} X$ and $c_{1} X, c_{1} X$ is said to be a extent of the compactification $c_{0} X$, denoted by $c_{0} X \leq_{K} c_{1} X$, if there is a continuous map $f: c_{1} X \rightarrow c_{0} X$ such that

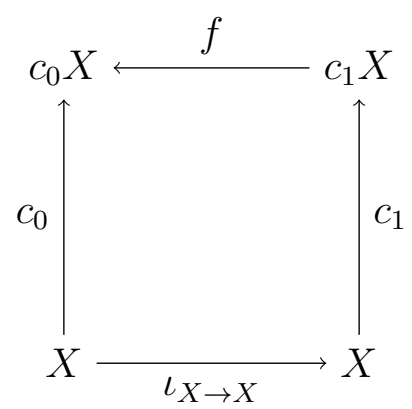


is a commutative diagram. In this convenction, given spaces $X$ and $Y$, where $Y$ is compact, if $g: X \rightarrow Y$ is continuous extendable over $c_{0} X$, say by $\bar{g}$, then $g$ is also continuous extendable over $c_{1} X$, namely by $G=f \circ \bar{g}$, i.e. any subdiagram of the following diagram is commutative:

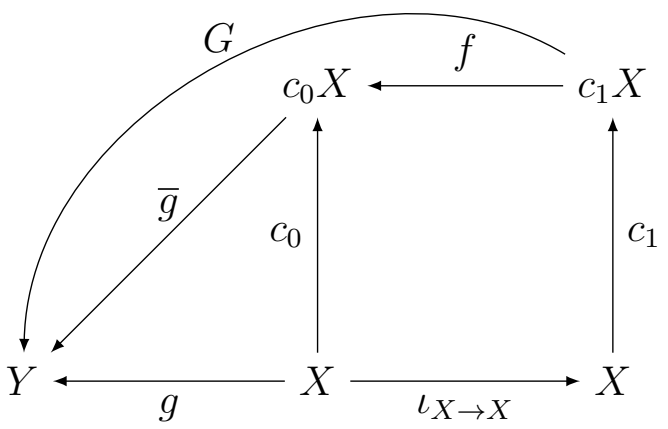

The Taĭmanov's Theorem 4.1.5 also proves the converse, i.e. if, for every continuous functions $f$ from $X$ to $Y$ that is continuous extentable over $c_{0} X, f$ is also continuous extendable over $c_{1} X$, then $c_{0} X \leq_{K} c_{1} X$. Indeed, if $F_{0}$ and $F_{1}$ are closed and disjoints subsets of $c_{0} X$, the normality of $c_{0} X$ produces a continuous function $f: c_{0} X \rightarrow \mathbf{I}$ such that, for every $i \in\{0,1\}, F_{i} \subseteq f^{-1}[\{i\}]$. By the hypothesis, there is a continuous function $g: c_{1} X \rightarrow \mathbf{I}$ such that $g \circ c_{1}=f \circ c_{0}$. Hence, by setting $h=c_{0} \circ c_{1}^{-1}: c_{1}[X] \rightarrow c_{0} X$, for each $i \in\{0,1\}$,

$$
\begin{aligned}
h^{-1}\left[F_{0}\right] & \subseteq h^{-1}\left[f^{-1}[\{i\}]\right]=\left(c_{0} \circ c_{1}\right)^{-1}\left[f^{-1}[\{i\}]\right] \subseteq \\
& \subseteq c_{1}\left[\left(f \circ c_{0}\right)^{-1}[\{i\}]\right]=c_{1}\left[\left(g \circ c_{1}\right)^{-1}[\{i\}]\right] \subseteq g^{-1}[\{i\}]
\end{aligned}
$$

Thus,

$$
\operatorname{cl}_{c_{1} X}\left(h^{-1}\left[F_{0}\right]\right) \cap \operatorname{cl}_{c_{1} X}\left(h^{-1}\left[F_{1}\right]\right)=\emptyset,
$$

testifying that $h$ has a continuous extension $H$ over $c_{1} X$. Obviuously, $c_{0}=H \circ c_{1}$.

Any compactifications $c_{0} X$ and $c_{1} X$ satisfying $c_{0} X \leq_{K} c_{1} X$ and $c_{1} X \leq_{K} c_{0} X$ must be homeomorphic. Indeed, let $f_{0}: c_{0} X \rightarrow c_{1} X$ and $f_{1}: c_{1} X \rightarrow c_{0} X$ be continuous functions such that any subdiagram of

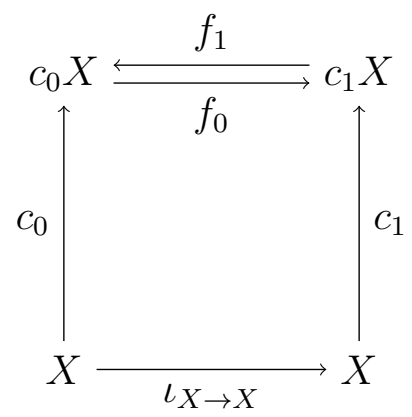

is commutative. Thus,

$$
\left(f_{0} \circ f_{1}\right) \circ c_{1}=f_{0} \circ\left(f_{1} \circ c_{1}\right)=f_{0} \circ c_{0}=c_{1},
$$


and

$$
\left(f_{1} \circ f_{0}\right) \circ c_{0}=f_{1} \circ\left(f_{0} \circ c_{0}\right)=f_{1} \circ c_{1}=c_{0}
$$

Hence,

$$
f_{0} \circ f_{1}\left\lceil_{c_{1}[X]}=\iota_{c_{1}[X]} \text { and } f_{1} \circ f_{0} \uparrow_{c_{0}[X]}=\iota_{c_{0}[X]} .\right.
$$

As, for every $i \in\{0,1\}, c_{i}[X]$ is dense in $c_{i} X, f_{0} \circ f_{1}=\iota_{c_{1} X}$ and $f_{1} \circ f_{0}=\iota_{c_{1} X}$, which proves that these both functions are bijective. Clearly, as $c_{0} X$ and $c_{1} X$ are compact, $f_{0}$ and $f_{1}$ are closed and thus these functions are homeomorphisms.

If $c X$ is a compactification of a Tychonoff space $X$, then $c$ separates points from closets sets. Thus, if $\left\langle c_{s} X_{s}\right\rangle_{s \in S}$ is a family of compactifications of $X$, then the map $c=\triangle_{s \in S} c_{s}: X \rightarrow \prod_{s \in S} c_{s} X_{s}$ is a homeomorphic embedding, which makes $c X=$ $\mathrm{cl}_{\prod_{s \in S} c_{s} X_{s}}[c[X]]$ a compactification of $X$. Now, the restriction of each projection $\mathrm{p}_{s}$ to $c X$ proves that $c_{s} X_{s} \leq_{K} c X$. Moreover, if $d X$ is a compactification of $X$ that, for each $s \in S$, produces a continuous function $f_{s}: d X \rightarrow c_{s} X_{s}$ fulfilling $f_{s} \circ d=c_{s}$, then the diagonal $f=\triangle_{s \in S} f_{s}$ satisfies $f \circ c=f_{s}$. Thus $c X$ is the supremum of the family $\left\langle c_{s} X_{s}\right\rangle_{s \in S}$ with respect to the order $\leq_{K}$ in $C(X)$.

The supremum of $\mathscr{K}(X)$ is called $\check{C}$ ech-Stone compactification of the space $X$ and a representative of the class of all mutually homeomorphic Cech-Stone compactifications of $X$ is denoted by $\beta X$.

4.2.1 Corolary. The Cech-Stone compactification $\beta X$ of a Tychonoff space $X$ has is topolocally caracterized by the following property: given any compact space $K$ and any continuous function $f: X \rightarrow K$, one can find a continuous function $F: \beta X \rightarrow K$ such that

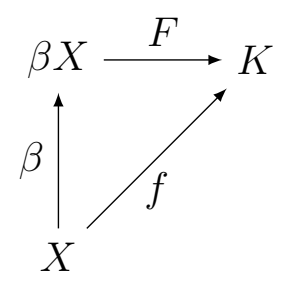

is a commutative diagram.

4.2.2 Lemma. Any compactification of a Tychonoff $X$ space whose closure operator disjoints completely separated sets of $X$ is equivalent to the Čech-Stone compactification of $X$.

Proof: The problem is obviously reducible to the case where the completely separated sets are functionally closed sets of $X$. In this particular case, the result is true by the application of 4.1.1.

4.2.3 Lemma. For a Tychonoff space $X, \beta X$ is precisely the unique compactification where the clousure operator is a lattice morfism from $\mathrm{FC}(X)$ to the set of all closed subsets of $\beta X$. 
Let $X$ be a Tychonoff space having more than one element; suppose that $\mathcal{L}$ is a sublattice of $\mathrm{FC}(X)$ that is also a normal base of closed subsets for $X$. Then, then $\lambda: X \rightarrow \operatorname{stn} \mathcal{L}$ is a continuous and injective function. Indeed, as

$$
\mathscr{B}=\{\{p: F \in p\}: F \in \mathcal{L}\}
$$

is a base of open subsets for $\operatorname{stn} \mathcal{L}$, for any $F \in \mathcal{L}$ and any point $x$ of $X$,

$$
x \in \lambda^{-1}[\{p \in \operatorname{stn} \mathcal{L}: F \in p\}]
$$

if and only if $x \in F$. As one could find a member of $\mathscr{F}$ that does not contain $x, \lambda$ is continuous. Moreover, as $\mathscr{F}$ is a normal base, $\lambda$ is a injective function and one can find an element of $\mathcal{B}$ that is a image under $\lambda$. Thus, $X$ cam be densely embedded into $\operatorname{stn} \mathcal{L}$. As such, $\operatorname{stn} \mathcal{L}$ is a compactification of $X$.

For any space $X$, let $\mathrm{C}(X)$ be the ring of all continuous real functions defined in $X$. Let $c X$ be a compactification of a space $X$. Then, consider

$$
\mathcal{L}=\left\{f \uparrow_{X}^{-1}[\{0\}]: f \in \mathrm{C}(c X)\right\} .
$$

4.2.4 Lemma. $\mathcal{L}$ is a sublattice of $\mathrm{FC}(X)$ that is also a normal base of closed subsets for $X$ that equates to $\mathrm{FC}(X)$ if and only if $c X=\beta X$; moreover, $\operatorname{stn} \mathcal{L}$ is homeomorphic to $c X$.

Proof: If $F_{0}$ and $F_{1}$ are elements of $\mathcal{L}$, say by the grace of $f_{0}, f_{1} \in \mathrm{C}(c X)$, respectively, then so are $F_{0} \cap F_{1}$ and $F_{0} \cup F_{1}$, for

$$
F_{0} \cap F_{1}=\left(f_{0} \Upsilon_{X}+f_{1} \Upsilon_{X}\right)^{-1}[\{0\}] \quad \text { and } \quad F_{0} \cup F_{1}=\left(f_{0} \Upsilon_{X} \cdot f_{1} \Upsilon_{X}\right)^{-1}[\{0\}],
$$

and

$$
f_{0} \uparrow_{X}+f_{1} \Upsilon_{X}=\left(f_{0}+f_{1}\right) \Upsilon_{X} \quad \text { and } f_{0} \Upsilon_{X} \cdot f_{1} \Upsilon_{X}=\left(f_{0} \cdot f_{1}\right) \Upsilon_{X} .
$$

Consequently, $\mathcal{L}$ is a sublattice of $\mathrm{FC}(X)$.

Now, if $x \in X$ and $F \subseteq X$ is a closed subset of $X$ that does not contain $x$, then, as $F \subseteq \mathrm{cl}_{c X} F$ and $c X$ is a Tychonoff space, one can find a continuous function $f: c X \rightarrow \mathbf{I}$ that separates $x$ and $F$. Thus, $f \uparrow_{X}^{-1}[\{0\}]$ is a member of $\mathcal{L}$ that contains $x$ and does not meet $F$. Lastly, if $G$ and $H$ are members of $\mathcal{L}$, say by testimony of the members $g$ and $h$ of $\mathrm{C}(c X)$, then

$$
G^{\prime}=(g \cdot h) \uparrow_{X}^{-1}\left[\left\{\frac{1}{3}\right\}\right] \quad \text { and } \quad F^{\prime}=(g \cdot h) \uparrow_{X}^{-1}\left[\left\{\frac{2}{3}\right\}\right]
$$

are disjoint members of $\mathcal{L}$ such that $G \subseteq X \backslash G^{\prime}$ and $H \subseteq X \backslash H^{\prime}$.

Now, if $Y$ is a compact space and $f: X \rightarrow Y$ is a continuous function, the function $g=f \circ \lambda^{-1}: \lambda[X] \rightarrow Y$ is continuous. Given two closed and disjoint subsets $F_{0}$ and $F_{1}$ of $Y$, the normality of $Y$ testifies the existence of two functionally closed and disjoint $G_{0}$ and $G_{1}$ such that, for any $i \in\{0,1\}, F_{i} \subseteq G_{i}$. Thus, as for every $i \in\{0,1\}, f^{-1}\left[G_{i}\right]$ is a functionally closed subset of $X$,

$$
\operatorname{cl}_{\operatorname{stn} \mathcal{L}} f^{-1}\left[G_{i}\right]=\left\{\mathfrak{p} \in \operatorname{stn} \mathcal{L}: f^{-1}\left[G_{i}\right] \in \mathfrak{p}\right\} .
$$


Therefore, no ultrafilter can contain the intersection $\left(\operatorname{cl}_{\operatorname{stn} \mathcal{L}} f^{-1}\left[G_{0}\right]\right) \cap\left(\operatorname{cl}_{\operatorname{stn} \mathcal{L}} f^{-1}\left[G_{1}\right]\right)$ which, with help of the Talmanov's theorem (4.1.5), proves that $f \circ \lambda^{-1}$ is continuous extendable over $\operatorname{stn} \mathcal{L}$ if and only if there is some $\bar{f} \in \mathrm{C}(c X)$ such that $f=\bar{f} \uparrow_{X}$. Hence, $\operatorname{stn} \mathcal{L}$ and $c X$ are homeomorphic for they extend the same continuous functions of $X$ and $\mathcal{L}=\mathrm{FC}(X)$ if and only if $\operatorname{stn} \mathcal{L}$ extends all functions of $\mathrm{C}^{*}(X)$.

4.2.5 Corolary. $\operatorname{stn} \mathrm{FC}(X)$ is $\beta X$.

Therefore, there is a biunivocal correspondence between the set of all compactifications of $X$, namely $\mathcal{K}(X)$, and the the set of all normal sublattices of $\mathrm{FC}(X)$. The property of being a normal sublattice of $\mathrm{FC}(X)$ is crucial to characterize all compactifications of a Tychonoff space $X$ in this fashion; indeed, for any cardinal $\kappa$ satisfying $2^{\kappa} \geq \omega_{\kappa}$, in [Ul'77], Ul'janov constructed an example of a compactification of $\mathbf{D}(\kappa)$ that does not comes from the Stone space of a non-sublattice of $\mathrm{FC}(X)$ that is a sublattice of $\wp(X)$ and a normal base of closed subsets for $X$.

\subsection{The Čech-Stone Compactification}

As seen previously, the $\check{C}$ ech-Stone compactification of a Tychonoff space $X$ is any compactification $c X$ of $X$ that, in a presence of a compact space $K$ and a continuous map $f: X \rightarrow K$, produces a continuous map $F: c X \rightarrow K$ so that

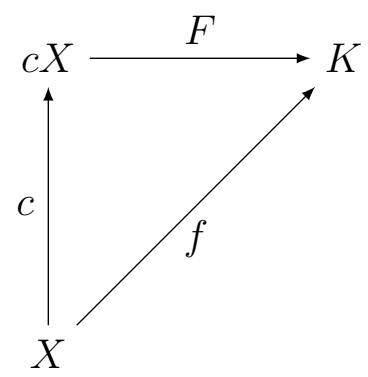

is a commutative diagram.

It's easy to see that, given any compactification $c_{0} X$ and a Čech-Stone compactification $c X, c_{0} X \leq_{K} c_{X}$. Thus all Čech-Stone compactifications of a Tychonoff space $X$ are mutually homeomorphic. Moreover, considering a transversal of the class of all compactifications of $X$ with respect of the equivalence relation generated by $\leq_{K}$, a representative of the equivalence class of the Čech-Stone compactifications of $X$, in this transversal, is the $\leq_{K}$-maximum.

As said in the previous chapter, $\beta X$ will denote a representative of the equivalence class of all Čech-Stone compactifications of a Tychonoff space $X$. For a proof of the following results, the reader can consult [Eng89].

As $\beta X$ can be constructed from the Stone space of the lattice $\mathrm{FC}(X)$,

$$
|\beta X|=|\operatorname{stn} \mathrm{FC}(X)| \leq 2^{|\mathrm{FC}(X)|} \leq 2^{\mathrm{w}(X)} .
$$


Hence, if $D$ is a discrete space of weight $\kappa$, then $|\beta D| \leq 2^{2^{\kappa}}$.

For any topological space $X$, one can define the cardinal

$$
\mathrm{d}(X)=\min \{|D|: D \text { is a dense subset of } X\}
$$

called density of $X$. It's easy to see that $\mathrm{d}(X) \leq \mathrm{w}(X)$.

4.3.1 Theorem. (Hewitt-Marczewski-Pondiczery) If $\left\langle X_{s}\right\rangle_{s \in S}$ is a collection of topological spaces and $\kappa$ is a cardinal such that, for any $s \in S, \mathrm{~d}\left(X_{s}\right) \leq \kappa$ and $|S| \leq 2^{\kappa}$, then

$$
\mathrm{d}\left(\prod_{s \in S} X_{s}\right) \leq \kappa
$$

In virtue of the the Hewitt-Marczewski-Pondeczery Theorem, $\mathbf{I}^{2^{\kappa}}$ has a dense subspace $M$ of cardinality $\kappa$. Thus, any continuous surjection of $D$ to $M$ must extends to a continuous surjection $f: \beta D \rightarrow \mathbf{I}^{2^{\kappa}}$. Thus, by the Cantor-Bernstein theorem, $|\beta D|=2^{2^{\kappa}}$. Particularly, $|\beta \mathbf{N}|=2^{\mathfrak{c}}$.

4.3.2 Lemma. Any compactification of a Tychonoff $X$ space whose closure operator disjoints completely separated sets of $X$ is homeomorphic to the Cech-Stone compactification of $X$.

Proof: The problem is obviously reducible to the case where the completely separated sets are functionally closed sets of $X$. In this particular case, the result is true by the application of 4.1.1.

4.3.3 Lemma. For a Tychonoff space $X, \beta X$ is precisely the unique compactification where the closure operator is a lattice morphism from $\mathrm{FC}(X)$ to the set of all closed subsets of $\beta X$.

4.3.4 Corolary. If $A \subseteq \beta \mathrm{N}$ is a countable and discrete, then $\mathrm{cl}_{\beta \mathbf{N}} A$ is homeomorphic to $\beta \mathbf{N}$

4.3.5 Lemma. Let $F$ be an infinite closed subset of $\beta \mathbf{N}$. Then, $F$ contains a copy of $\beta \mathbf{N}$.

Proof: As $F$ is a infinite Hausdorff space, there is a discrete countable

$$
A=\left\{x_{n}: n \in \mathbf{N}\right\} \subseteq F
$$

that, in the presence of (4.3.4), has a copy of $\beta \mathbf{N}$ in its closure.

Let $c X$ be any compactification of a Tychonoff space $X$. Then the set $c X \backslash X$ is called the reminder of the compactification $c X$. In particular, the reminder of $\beta X$ is denoted by $X^{*}$.

4.3.6 Theorem. [Č59; GJ60] Any non-empty $\mathrm{G}_{\delta}$ set of the Čech-Stone compactification of a non-compact Tychonoff space $X$ contained in the remainder $X^{*}$ has a copy of $\mathbf{N}$. 
Proof: Let $G$ be a $\mathrm{G}_{\delta}$ of $\beta X$; as $\beta X$ is a normal space, there is a continuous function $f: \beta X \rightarrow \mathbf{I}$ such that $G=f^{-1}[\{0\}]$. Thus any point contained in $X$ has a positive image under $f$. Moreover, $X$ is a dense subspace of $\beta X$, inductively one can construct a $\left\langle x_{n}\right\rangle_{n \in \mathbf{N}}$ within $X$ such that

$$
f\left(x_{n}\right) \leq \frac{1}{n+1} \quad \text { and } \quad f\left(x_{n}\right)>f\left(x_{n+1}\right) .
$$

Thus, being $A=\left\{x_{n}: n \in \mathbf{N}\right\}, A$ is discrete; thus, any two mutually disjoint and closed subsets $F_{0}$ and $F_{1}$ of $A$ have closed and mutually disjoint images under $f$; the normality of $] 0,1]$ testify that $F_{0}$ and $F_{1}$ are completely separated in $\left.] 0,1\right]$ and, via $f$, also in $X$. As $\mathrm{FC}(A)=\wp(A)$, the lemma (4.3.3) proves that $\mathrm{cl}_{\beta X}$ is $\beta N$. Moreover,

$$
\mathrm{cl}_{\beta X}(A)=\bigcup_{\mathfrak{p} \in \beta \mathbf{N}} p-\lim x_{n}
$$

Thus $\operatorname{cl}_{\beta X} A \backslash A \subseteq G$, for $f\left(p-\lim x_{n}\right)=p$ - $\lim f\left(x_{n}\right)$, whenever $\mathfrak{p} \in \beta \mathbf{N}$; hence that $G$ has a copy of $\mathbf{N}^{*}$. As any closed and infinite subset of $\beta \mathbf{N}$ has a copy of $\beta \mathbf{N}$, the proof is complete.

4.3.7 Corolary. No point of the reminder of the Čech-Stone compactification of a Tychonoff space can be a $\mathrm{G}_{\delta}$.

4.3.8 Corolary. Any space $X$ that is a countable union of compact subspaces has a remainder with no isolated points.

4.3.9 Lemma. For a Tychonoff space $X$, the following is equivalent

(4.3.9.1). $X$ is locally compact;

(4.3.9.2). $X$ is open in any of its compactification;

(4.3.9.3). $X$ is open in $\beta X$.

If $D$ is a discrete space, then for any $A \subseteq D$,

$$
\operatorname{cl}_{\beta D}(A)=\{\mathfrak{p} \in \beta D: A \in \mathfrak{p}\} .
$$

Thus, one can define the map $(\cdot)^{*}: \wp(D) \rightarrow \wp(\beta D)$ by

$$
A^{*}=\operatorname{cl}_{\beta D}(A) \backslash A \text {. }
$$

Evidently, $A^{*}=\emptyset$ if and only if $A$ is a compact subspace of $D$, i.e. $A^{*}=\emptyset$ if and only if $A$ is finite.

4.3.10 Lemma. For any subsets $A$ and $B$ of a discrete space $D, A^{*}=B^{*}$ if and only if $A \backslash B$ and $B \backslash A$ are finite.

Thus, $(\cdot)^{*}$ is a boolean morphism from $\wp(D)$ to $\operatorname{CO}(\beta D)$ whose kernel (i.e. the elements whose value of $(\cdot)^{*}$ is null) is $[D]^{<\omega}$. Thus, a usual algebraic argumentation proves that $\operatorname{CO}(\beta D \backslash D)$ is isomorphic to $\wp(D) /[D]^{<\omega}$. Hence, $D^{*}$ is a compact zerodimensional space with no isolated points that is homeomorphic to the Stone space of the Boolean algebra $\wp(D) /[D]^{<\omega}$. 


\section{Part II}

Algebraic Topological Dynamics 


\section{CHAPTER 5}

\section{Introductory Concepts}

\section{$5.1 \quad$ Semigroup structure of $\beta \mathrm{N}$}

A semigroup is a pair $\langle S, \cdot\rangle$ in which $S$ is non-empty set and - is a associative binary operation on $S$. Henceforth, a semigroup will be denoted only by its set, where the operation will be understood from the context.

Allow $S$ to be a semigroup whose operation is $*$ and furnished with the discrete topology; then $\beta S$ will be the Cech-Stone compactification of $S$, i.e. the collection of all ultrafilters of $\wp(S)$ equipped with the zero-dimensional topology whose base for openand-closed sets is given by

$$
\mathrm{CO}(\beta S)=\{\{p \in \beta S: A \in p\}: A \in \wp(S)\} .
$$

Given any $s \in S$, let

$$
\begin{aligned}
l_{s}: S & \rightarrow \beta S \\
t & \mapsto s * t
\end{aligned} \text { and } \quad \begin{array}{rlrl}
r_{s}: S & \rightarrow \beta S \\
& t & \mapsto t * s .
\end{array}
$$

Obviously both $l_{s}$ and $r_{s}$ are continuous functions - since $S$ is discrete - and thus there are unique $\bar{l}_{s}, \bar{r}_{s}: \beta S \rightarrow \beta S$ that continuously extends $l_{s}$ and $r_{s}$ over $\beta S$, respectively. Additionally,

$$
\bar{l}_{s}(p)=p-\lim _{t \in S} l_{s}(t)=\lim _{t \rightarrow p} l_{s}(t)
$$

and

$$
\bar{r}_{s}(p)=p-\lim _{t \in S} r_{s}(t)=\lim _{t \rightarrow p} r_{s}(t) .
$$

Fixed a $p \in \beta S$, consider the functions

$$
\begin{aligned}
& L_{p}: S \rightarrow \beta S \\
& s \mapsto \bar{l}_{s}(p) \text { and } \\
& R_{p}: S \rightarrow \beta S \\
& s \longmapsto \bar{l}_{s}(p) \quad s \mapsto \bar{r}_{s}(p) .
\end{aligned}
$$


As $S$ is discrete, $L_{p}$ and $R_{p}$ are continuous. Thus, by the property that characterizes $\beta S$, there exist continuous functions $\bar{L}_{p}, \bar{R}_{p}: \beta S \rightarrow \beta S$ which extend $L_{p}$ and $R_{p}$ over $\beta S$, respectively. Moreover, as $S$ is dense in $\beta S$, the continuity of both these functions testifies that, for every ultrafilters $p$ and $q$ of $\wp(S), \bar{L}_{p}(q)=\bar{R}_{q}(p)$. Therefore, the operation $\star: \beta S \times \beta S \rightarrow \beta S$ given by $p \star q=\bar{L}_{q}(q)$ is left and right continuous and uniquely extends of $*$ over $\beta S$.

Observe that, for any ultrafilters $p, q$ and $r$,

$$
\begin{aligned}
(p \star q) \star r & =p-\lim _{s \in S} q-\lim _{t \in S} r-\lim _{u \in S}((s * t) * u) \stackrel{1}{=} p-\lim _{s \in S} q-\lim _{t \in S} r-\lim _{u \in S}(s *(t * u)) \stackrel{2}{=} \\
& =p-\lim _{s \in S} s \star\left(q-\lim _{t \in S} r-\lim _{u \in S}(t * u) \stackrel{3}{=} p \star\left(q-\lim _{t \in S} t \star r-\lim _{u \in S} u\right)=\right. \\
& =p \star(q \star r) .
\end{aligned}
$$

where

- in equality (1), is used the associativity of $*$;

- in equality (2), is used the continuity of $\bar{L}_{s}$; and

- in equality (3), is used the continuity of $\bar{L}_{t}$

5.1.1 Lemma. There is a unique associative operation $\star$ on $\beta S$ such that

ESO1 $\star$ extends $*$, i.e. given any $s, t \in S, s \star t=s * t$;

ESO2 $\star$ is left and right continuous, i.e. for each $p \in \beta S$, the functions $\lambda_{p}, \rho_{p}$ : $\beta S \rightarrow \beta S$ given by $\lambda_{p}(q)=p \star q$ and $\rho_{p}(q)=q \star p$ are continuous;

ESO4 given any compact left-and-right-topological semigroup ${ }^{1}\langle c S, \bullet\rangle$ such that $c S$ is a compactification of $S$ and $\bullet$ satisfies the relativisation of ESO1 and ESO2 to $\bullet$ there is a continuous semigroup morphism $f: \beta S \rightarrow c S$ that makes the following diagram commutative

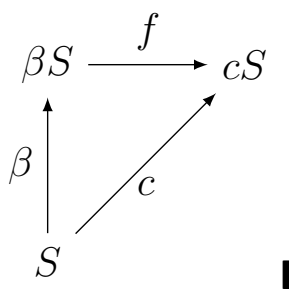

${ }^{1}$ A left-topological semigroup is a semigroup $\langle S, *\rangle$ together with a topology $\tau$ that, for every $s \in S$, makes the function

$$
\begin{aligned}
\lambda_{s}: S & \rightarrow S \\
t & \mapsto s * t
\end{aligned}
$$

continuous. Analogously, one can define the notion of a right-topological semigroup. Unfortunately, a left-topological semigroup that also happens to be a right-topological semigroup can fail to be a topological semigroup, i.e. $*$ might not be continuous on $S \times S$; this is the case of $\beta \mathbf{N}$. 
Henceforth, a semigroup $S$ (for instance $\mathbf{N}$ or $\mathbf{Z}$ ) will always carry the discrete topology; considering this space, $\beta S$ will be the Cech-Stone compactification in which the extension of the operation defined in $S$ will be as in 5.1.1.

Hence, one can consider the operations,$+ \cdot$ of $\beta \mathbf{N}$ that are right and left continuous and uniquely extend the usual operations of addition and multiplication of $\mathbf{N}$, respectively.

The extension of a discrete semigroup operation over its Čech-Stone semigroup compactification can be obtained directly:

5.1.2 Lemma. Let $\langle S, *\rangle$ a discrete semigroup and consider the semigroup operation $\star$ on $\beta S$ as in 5.1.1. Then, given $p, q \in \beta S$,

$$
p \star q=\{A \subseteq S:\{s \in S:\{t \in S: s * t \in A\} \in q\} \in p\} .
$$

Proof: Given any $A \subseteq S, A \in p \star q$ if and only if $p \star q \in \operatorname{cl}_{\beta S} A . \operatorname{As~cl}_{\beta S} A$ is a open-and-closed in $\beta S$ and

$$
p \star q=p-\lim _{s \in S} q-\lim _{t \in S}(s * t),
$$

the definition of p-limits proves that $A \in p \star q$ if and only if

$$
\left\{s \in S: q-\lim _{t \in S}(s * t) \in \operatorname{cl}_{\beta S} A\right\} \in q,
$$

which will occur if and only if

$$
\left\{s \in S:\left\{t \in S:(s * t) \in \operatorname{cl}_{\beta S} A\right\} \in q\right\} \in p .
$$

Now, $s * t$ is always member of $S$, which implies that $s * t$ will always - in this case be a member of $A$; thus $A \in p \star q$ if and only if

$$
A \in\{B \subseteq S:\{s \in S:\{t \in S: s * t \in A\} \in q\} \in p\}
$$

as desired.

5.1.3 Corolary. For any two ultrafilters $p$ and $q$ of $\wp(\mathbf{N})$

(5.1.3.1). $p+q=\{A \subseteq \mathbf{N}:\{n \in \mathbf{N}:\{m \in \mathbf{N}: n+m \in A\} \in q\} \in p\}$; and

(5.1.3.2). $p \cdot q=\{A \subseteq \mathbf{N}:\{n \in \mathbf{N}:\{m \in \mathbf{N}: n \cdot m \in A\} \in q\} \in p\}$.

As in the development of group theory, the centre of a semigroup is the collection of all elements within this semigroup that commute with any elements of this semigroup. For any $p \in \beta \mathbf{N}$ and $n \in \mathbf{N}$, the continuity of $\bar{R}_{n}$ and $\bar{L}_{n}$ testifies that

$$
p+n=\bar{R}_{n}(p)=p-\lim _{m \in \mathbf{N}} \bar{R}_{n}(m)=p-\lim _{m \in \mathbf{N}} \bar{L}_{n}(m)=n+p .
$$


Thus, $\mathbf{N}$ is inscribed in the centre of $\langle\beta \mathbf{N},+\rangle$. A similar argument shows that $\mathbf{N}$ is also inscribed into the centre of $\langle\beta \mathbf{N}, \cdot\rangle$. It will be seen in 6.4 .2 that $\mathbf{N}$ is actually the centre of $\langle\beta \mathbf{N},+\rangle$ and $\langle\beta \mathbf{N}, \cdot\rangle$.

Let $S$ be a semigroup and $A \subseteq S$; for any $s \in S$, let

$$
A+s=\{a * s: a \in A\} .
$$

If $B$ is a subset of $S$, then

$$
A+B=\{a+b: a \in A \wedge b \in B\}=\bigcup_{b \in B} A+b .
$$

5.1.4 Lemma. Let $\langle S, *\rangle$ be a topological left semigroup. The following propositions are true

(5.1.4.1). If $A, B \subseteq S$, then $\operatorname{cl}_{S}(A)+B \subseteq \operatorname{cl}_{S}(A+B)$;

(5.1.4.2). If $S$ is compact, for every $A \subseteq S$ and $s \in S, \operatorname{cl}_{S}(A)+s=\operatorname{cl}_{S}(A+s)$;

(5.1.4.3). If $A, B \subseteq S$ and $A$ is contained in the centre of $S$, then $\operatorname{cl}_{S}(A)+\operatorname{cl}_{S}(B) \subseteq$ $\operatorname{cl}_{S}(A+B)$.

Proof: As $S$ is a left topological semigroup, for each $s \in S$, the function $r_{s}: S \rightarrow S$ given by $\rho_{s}(t)=t * s$ is continuous. Hence for every $s \in B$, the set $\rho_{s}^{-1}\left[\operatorname{cl}_{S}(A+s)\right]$ is a closed subset of $S$ which contains $A$. Hence,

$$
\operatorname{cl}_{S}(A)+s \subseteq \operatorname{cl}_{S}(A+s) \subseteq \operatorname{cl}_{S}(A+B) .
$$

Since

$$
\bigcup_{s \in B}\left(\mathrm{cl}_{S}(A)+s\right)=\mathrm{cl}_{S}(A)+B
$$

$\mathrm{cl}_{S}(A)+B$ must be a subset of $\mathrm{cl}_{S}(A+B)$.

For each $s \in S$, one can prove that $\mathrm{cl}_{S}(A)+s=\rho_{s}\left[\mathrm{cl}_{S}(A)\right]$; as $S$ is compact and $\rho_{s}$ is continuous, it can be deduced that $\mathrm{cl}_{S}(A)+s$ is compact and thus a closed subset of $S$. As $A+b \subseteq \operatorname{cl}_{S}(A)+b$, it follows that $\operatorname{cl}_{S}(A)+b=\operatorname{cl}_{S}(A+b)$, which settles (5.1.4.2).

For the last proposition, the validity of $(\mathbf{5 . 1 . 4 . 1})$ proves that

$$
\operatorname{cl}_{S}(A)+\operatorname{cl}_{S}(B) \subseteq \operatorname{cl}_{S}\left(A+\operatorname{cl}_{S}(B)\right) ;
$$

since $A$ is a subset inscribed in the centre of $S, A+\operatorname{cl}_{S}(B)=\operatorname{cl}_{S}(B)+A$. Thus, using again (5.1.4.1),

$$
\operatorname{cl}_{S}(A)+\operatorname{cl}_{S}(B) \subseteq \operatorname{cl}_{S}\left(A+\operatorname{cl}_{S}(B)\right)=\operatorname{cl}_{S}\left(\operatorname{cl}_{S}(B)+A\right) \subseteq \operatorname{cl}_{S}(B+A)=\operatorname{cl}_{S}(A+B),
$$

concluding the proof. 


\subsection{Ideals in $\beta \mathrm{N}$}

A left ideal of a semigroup $\langle S, *\rangle$ is a non-empty subset $L$ of $S$ such that, given $l \in L$ and $s \in S, s * l \in L$; analogously, one can define the notion of a right ideal of $S$. A non empty subset $I$ of $S$ that are both left and right ideal of $S$ is called an ideal of $S$. Evidently, a non empty subset $L$ of $S$ is a left ideal if and only if $S * L \subseteq L$; analogous relations also follows for right ideal and ideals of $S$.

The (left or right) ideals of a semigroup $S$ shares some known properties of an ideal of a ring, such as:

5.2.1 Lemma. Let $S$ be a semigroup. Then, the following properties are true:

(i) if $L_{0}$ and $L_{1}$ are (left or right) ideals of $S$, then $L_{0} \cap L_{1}$ is an (respectively left or right) ideal of $S$ if and only if their intersection is not empty;

(ii) the intersection between an left and a right ideal of $S$ is always non empty; and

(iii) if $x \in S$, then $x S$ is a right ideal of $S, S x$ is a left ideal of $S$ and $x S x$ is an ideal of $S$;

Let $S$ be a semigroup and $p \in \beta S$. If $\lambda_{p}: \beta S \rightarrow \beta S$ is given by $\lambda_{p}(q)=q+p$. Then,

$$
L=\lambda_{p}[\beta S]=\beta \mathbf{N}+p
$$

is a left ideal of $\langle\beta \mathbf{N},+\rangle$. As $\beta S$ is compact and $\lambda_{p}$ is continuous, $L$ is also compact. Moreover, if $S$ has a $\subseteq$-minimal ideal $M$, given any ideal $I$ of $S$, by the item (ii) of 5.2.1, one can prove that $M \cap I \neq \emptyset$; thus the ideal $M \cap I$ is $M$, for $M$ is minimal. Therefore, a semigroup $S$ has at most one $\subseteq$-minimal ideal; in such cases, $K(S)$ will denote the $\subseteq$-minimal ideal of $S$.

The lemma 5.2.2 suggest the existence of a special class of ultrafilters inside $\beta S$ that generates minimal left ideals; $\mathrm{m} K(\beta S)$, if it exists will be those ultrafilters that will generate $K(\beta S)$. Observe, however, that this is quite different from the notion of principality of ideals in classical algebra. Indeed, any compact infinite set of $\beta S$ should contain at least a copy of $\beta \mathbf{N}$ and any ultrafilter present in this set will generate such ideals.

5.2.2 Lemma. Let $S$ be a semigroup and $M$ be a left ideal of $\beta S$. Then $M$ is $\subseteq$-minimal among the left ideals of $\beta S$ if and only if, for any $p \in M, M=\beta S \star p$. Analogously, a right ideal $M$ of $\beta S$ is $\subseteq$-minimal among the right ideals of $\beta S$ if and only if, for any $p \in M, M=p \star \beta S$.

Proof: Firstly, suppose that $M$ is $\subseteq$-minimal among the left ideals of $\beta S$. Given any $p \in M$, the fact that $M$ is a left ideal of $\beta S$ proves that $\beta S \star p \subseteq M$; thus the minimality of $M$ testifies for the desired equality.

Conversely, suppose that, for any $p \in M$ one has $M=\beta S \star p$; if $L$ is a left ideal of $\beta S$ that is contained in $M$, then given any $p \in L$,

$$
M=\beta S \star p \subseteq L \subseteq M ;
$$


which proves that $L=M$.

5.2.3 Corolary. Minimal (left, right or two sided) ideals of $\beta S$ are compact.

Proof: It's sufficient to see that the operation $\star$ is left and right continuous.

5.2.4 Corolary. Let $S$ be a semigroup; then every left ideal of $\langle\beta \mathbf{N},+\rangle$ contain a minimal left ideal.

Proof: Consider a left ideal $L$ of $\langle\beta S, \star\rangle$; then, for any $p \in L, \beta S \star p$ is a closed left ideal of $\beta S$ contained in $L$. Thus, the collection $\mathcal{L}$ of all closed left ideals of $\beta S$ that are contained in $L$ is not empty. Declare $\preceq$ to be the order defined in $\mathcal{L}$ by $I_{1} \preceq I_{0}$ if and only if $I_{0} \subseteq I_{1}$ and let $C$ be a $\preceq$-linearly ordered subset of $\mathcal{L}$. As $C$ has the FIP, clearly $\bigcap \subset \neq \emptyset$; moreover, given any $p$ present in such intersection, $\beta S \star p$ will be contained in any element of $C$, proving that $\bigcap \mathcal{C}$ is a closed left ideal of $\beta S$ that is contained in $L$. Hence, $\bigcap e$ is a $\preceq$-majorant for $C$. As such, the Kuratowski-Zorn Lemma produces a $\preceq$-maximal element $M \in \mathcal{L}$ that is, by the definition of $\preceq$, a $\subseteq$-minimal element $M$ of $\mathcal{L}$. Evidently, until now, there is no evidence that $M$ is a minimal left ideal of $\beta S$.

To prove that $M$ is a minimal left ideal of $\beta S$, let $p \in M$. Then, $\beta S \star p$ is a closed left ideal of $\beta S$ that is contained in $M$ and thus in $L$; thus, $\beta S \star p$ is a member of $\mathcal{L}$. As follows, the $\subseteq$-minimality of $M$ among the elements of $\mathcal{L}$ completes the proof.

5.2.5 Corolary. For a semigroup $S$, let $M$ be a minimal left ideal of $\beta S$. Then, for any $p \in M$ and $q \in \beta S$, there is a $r \in \beta S$ such that

$$
r \star q \star p=p .
$$

Proof: As $M$ is minimal and $q \star p \in M, M=\beta S \star(q \star p)$. Thus, as $p \in M$, one can find a $r \in \beta S$ such that $r \star q \star p=p$.

5.2.6 Theorem. Let $\mathcal{L}$ be the collection of all minimal left ideals of $\beta S$. Then,

$$
K(\beta S)=\bigcup \mathcal{L}
$$

Proof: Let $M=\bigcup \mathcal{L}$. For any $p \in M$, one can encounter a $L \in \mathcal{L}$ such that $p \in L ;$ as such,

$$
\beta S \star p=L \subseteq M
$$

To prove that $M$ is also a right ideal of $\beta S$, observe that it's sufficient to prove that, for any $q \in \beta S$ and $p \in M, \beta S \star(p \star q)$ is a minimal left ideal of $\beta S$. Indeed, if $\beta S \star(p \star q)$ is a minimal left ideal of $\beta S$, then $\beta S \star(p \star q) \subseteq M$ which proves that $p \star q \in M$.

To prove that $\beta S \star(p \star q)$ is a minimal left ideal of $\beta S$, one can use 5.2 .2 ; i.e. for any $r \in \beta S$,

$$
\beta S \star(r \star(p \star q))=\beta S \star(p \star q) .
$$


Indeed, by 5.2 .5 , one can find a $s \in \beta S$ such that

$$
s \star r \star p=p .
$$

Thus, by the associativity of $\star$,

$$
s \star(r \star p \star q)=(s \star r \star p) \star q=p \star q .
$$

It follows that $p \star q \in \beta S \star(r \star(p \star q))$. Thus, given any $t \in \beta S$, one can find a $s \in \beta S$ such that

$$
t \star(p \star q)=(t \star s) \star(r \star(p \star q)) .
$$

Lastly, let $I$ be any ideal of $\beta S$. Then, by the item (i) and (ii) of $5.2 .1, I \cap M$ is a left ideal of $\beta S$. Thus, the minimality of $M$ shows that $M \subseteq I \cap M \subseteq I$.

5.2.7 Corolary. There is an unique minimal ideal of $\beta S$.

5.2.8 Lemma. $\mathbf{N}^{*}$ is an ideal of $\langle\beta \mathbf{N},+\rangle$.

Proof: For every $n \in \mathbf{N}$, let $A_{n}$ be the set of all natural numbers greater than or equal to $n$. Then, for every natural number $m<n$, the fact that $\{m\}$ is isolated in $\beta \mathbf{N}$ testifies that $\{0, \ldots, n-1\}=\beta \mathbf{N} \backslash \operatorname{cl}_{\beta \mathbf{N}} A_{n}$. Thus, $\mathbf{N}^{*}=\bigcap_{n \in \mathbf{N}} \operatorname{cl}_{\beta \mathbf{N}} A_{n}$. As, for any $k \in \mathbf{N}, k+A_{n} \subseteq A_{n}$,

$$
k+\mathbf{N}^{*}=k+\bigcap_{n \in \mathbf{N}} \operatorname{cl}_{\beta \mathbf{N}} A_{n}=\bigcap_{n \in \mathbf{N}}\left(k+\operatorname{cl}_{n \in \mathbf{N}} A_{n}\right) \subseteq \bigcap_{n \in \mathbf{N}} \operatorname{cl}_{n \in \mathbf{N}} A_{n}=\mathbf{N}^{*} .
$$

The result thus follows from the fact that $\mathbf{N}^{*}$ is closed in $\beta \mathbf{N}$.

Evidently, the same argument can be applied to show that $\mathbf{N}^{*}$ is a right ideal of $\langle\beta \mathbf{N},+\rangle$.

5.2.9 Lemma. If $R$ is a right ideal of $\langle\beta \mathbf{N},+\rangle$, then $\operatorname{cl}_{\beta \mathbf{N}} R$ is a right ideal of $\langle\beta \mathbf{N},+\rangle$.

Proof: Let $p \in \mathrm{cl}_{\beta \mathbf{N}} R$ and $q \in \beta \mathbf{N}$. Given any $A \in p+q$,

$$
\{m \in \mathbf{N}:\{n \in \mathbf{N}: n+m \in A\} \in p\} \in q .
$$

Thus, one can pick a $m \in \mathbf{N}$ such that $\{n \in \mathbf{N}: n+m \in A\} \in p$; moreover, the closure of $\{n \in \mathbf{N}: n+m \in A\}$ will be a neighbourhood of $p$ in $\beta \mathbf{N}$. Hence there is a ultrafilter $r \in \operatorname{cl}_{\beta \mathbf{N}}(\{n \in \mathbf{N}: n+m \in A\}) \cap R$ so that $A ß r+m$. Clearly, $r+m \in \operatorname{cl}_{\beta \mathbf{N}}(A) \cap R$, which proves that $p+q \in \operatorname{cl}_{\beta \mathbf{N}} I$, for $\operatorname{cl}_{\beta \mathbf{N}} A$ is a neighbourhood of $p+q$.

Analogously, one can prove the following:

5.2.10 Lemma. If $L$ is a left ideal of $\langle\beta \mathbf{N},+\rangle$, then $\operatorname{cl}_{\beta \mathbf{N}} L$ is a left ideal of $\langle\beta \mathbf{N},+\rangle$. 


\subsection{Idempotents in $\beta \mathbf{N}$}

An idempotent element of a semigroup $\langle S, \star\rangle$ is a $x \in S$ such that $x \star x=x$. In the present section, the Ellis-Namakura theorem is presented. This theorem rises the opportunity of finding an idempotent element inside both $\langle\beta \mathbf{N},+\rangle$ and $\langle\beta \mathbf{N}, \cdot\rangle$.

5.3.1 Theorem. [Ell58; Num52] Every compact left-topological semigroup $\langle S, *\rangle$ has an idempotent element.

Proof: Let $\mathscr{I}$ be the collection of all non empty and closed subsets of $S$ that are invariant under $*$, i.e. all closed subsets $I$ of $S$ such that $I * I \subseteq I$. Consider the inverse containment relation $\supseteq$ in $\mathscr{I}$ and let $\mathcal{L}$ a $\supseteq$-linear subset of $\mathscr{I}$. Clearly, using the FIP, $I=\bigcap \mathcal{L}$ is a non empty closed subset of $S$ that clearly is also invariant under $*$. Hence, the Kuratowski-Zorn Lemma produces a $\supseteq$-maximal $M$ (that will be $\subseteq$-minimal) element of $\mathscr{I}$.

Given any $x \in M$ and $I \in \mathscr{I}$, by the left-continuity of + and the compactness of $I$ proves that $x+I$ is compact. Moreover, as $M$ is minimal of $\mathscr{I}$ and invariant under ,$+ M+x=M$. Thus, $M^{\prime}=\{y \in M: y+x=x\}$ is non empty, compact by the left-continuity of + and, by the associativity of + , also invariant under + . Therefore $M^{\prime}=M$, which shows that $x \in M^{\prime}$ and, consequently, $x$ is a idempotent element of $S$.

Cardinality will not play an important hole here, but, for the sake of information, in the case of $\beta \mathbf{N}$, the proof of the Ellis-Nakamura theorem can be modified to show that are $2^{\mathfrak{c}}$ idempotents of $\beta \mathbf{N}$, since any infinite compact subset of $\beta \mathbf{N}$ contains a copy of $\beta \mathbf{N}$.

As $\mathbf{N}^{*}$ is a closed ideal of $\langle\beta \mathbf{N},+\rangle$ and $\langle\beta \mathbf{N}, \cdot\rangle$, one can easily prove the following:

5.3.2 Corolary. There exists an ultrafilter inside $\beta \mathbf{N} \backslash \mathbf{N}$ that is idempotent with respect the operation of addition and multiplication.

\subsection{Syndetic Sets and the van der Waerden Theorem}

The following result is know as the van der Waerden Theorem:

5.4.1 Theorem. ([vdW27]) Let $A_{0}, \ldots, A_{k-1}$ be a non empty list of pairwise disjoint subsets of $\mathbf{N}$ such that $\mathbf{N}=\bigcup_{i<k} A_{i}$. Then, there is an $i<k$ such that $A_{i}$ contains arbitrary long arithmetic progressions.

Let $A_{0}, \ldots, A_{k-1}$ be as in (5.4.1). Then,

$$
\beta \mathbf{N}=\bigcup_{i<k} \operatorname{cl}_{\beta \mathbf{N}}\left(A_{i}\right) .
$$


By setting

$$
\left.\mathscr{A} \mathscr{P}=\left\{p \in \beta \mathbf{N}: \forall A \in p \forall k \in \mathbf{N} \exists a, b \in \mathbf{N}\left(\operatorname{AP}_{k}(a, b) \subseteq A\right)\right)\right\}
$$

one can easily see that (5.4.1) is a consequence of the existence of some $p \in \mathscr{A} \mathscr{P}$. Indeed, if there is some $p \in \mathscr{A} \mathscr{P}$, as $p$ is prime, some $A_{i}$ has to be a member of $p$. The hardest part is to prove that $\mathscr{A} \mathscr{P}$ is indeed not empty. Before proving that, let observe that $K(\beta \mathbf{N},+)$ and $K(\beta \mathbf{N}, \cdot)$ are contained in $\mathscr{A} \mathscr{P}$, for $\mathscr{A} \mathscr{P}$ is an ideal of $\langle\beta \mathbf{N},+\rangle$ and $\langle\beta \mathbf{N}, \cdot\rangle$.

5.4.2 Lemma. If $\mathscr{A} \mathscr{P} \neq \emptyset$, then $\mathscr{A} \mathscr{P}$ is a closed ideal of both $\langle\beta \mathbf{N},+\rangle$ and $\langle\beta \mathbf{N}, \cdot\rangle$.

Proof: Let $p$ be an element of $\mathscr{A} \mathscr{P}$ and $q$ be any element of $\beta \mathbf{N}$.

Then $\mathscr{A} \mathscr{P}$ is a left ideal of $\langle\beta \mathbf{N},+\rangle$ if and only if, for any $A \in p+q, A$ contains arbitrarily long arithmetic sequences; if $A \in p+q$, then is possible to find a $m \in \mathbf{N}$ such that

$$
A_{m}=\{n \in \mathbf{N}: m+n \in A\}
$$

is a member of $p$, for, by the set-theoretic definition of + ,

$$
\{m \in \mathbf{N}:\{n \in \mathbf{N}: m+n \in A\} \in p\} \in q .
$$

As $p \in \mathcal{A} \mathscr{P}$, for any $k \in \mathbf{N}$, one can find natural numbers $a$ and $b>0$ such that $\operatorname{AP}_{k}(a, b) \subseteq A_{m}$. Thus, $\operatorname{AP}_{k}(m+a, b) \subseteq A$, which testifies that $p+q$ is an element of $\mathcal{A} \mathscr{P}$.

Now, $\mathscr{A} \mathscr{P}$ is a left ideal of $\langle\beta \mathbf{N}, \cdot\rangle$ if and only if, for any element $A$ of $p \cdot q, A$ contains arbitrary long arithmetic progression. Let $A \in p \cdot q$; by the set-theoretic definition of the product of ultrafilters, there is a $m \in \mathbf{N}$ such that

$$
A_{m}=\{n \in \mathbf{N}: n \cdot m \in A\}
$$

is a member of $p$; as such, for any $k \in \mathbf{N}$, one can find $a, b \in \mathbf{N}$ satisfying $b>0$ and $\operatorname{AP}_{k}(a, b) \subseteq A_{m}$. Hence, $\operatorname{AP}_{k}(a \cdot m, b \cdot m) \subseteq A$.

The set $\mathscr{A} \mathscr{P}$ is a right ideal of $\langle\beta \mathbf{N},+\rangle$ if and only if, for any $A \in q+p, A$ contains arbitrarily long arithmetical progressions. But, by the set-theoretic definition of ,$+ A \in q+p$ if and only if

$$
\{n \in \mathbf{N}:\{m \in \mathbf{N}: n+m \in A\} \in p\} \in q .
$$

Thus, is possible to find an infinitude of $n \in \mathbf{N}$ for which the set $A_{n}=\{m \in \mathbf{N}: n+m \in$ A ) contains arbitrarily long arithmetic progressions, for $p$ is a member of $\mathscr{A} \mathscr{P}$; i.e. give any $k \in \mathbf{N}$, is possible to find naturals $a$ and $b$ such that $b>0$ and $\operatorname{AP}_{k}(a, b) \subseteq A_{n}$. As such, for every $t<k, n+(a+t \cdot b)$ is a member of $A$. As $q$ is an ultrafilter, is possible to find a $n_{0}$ such that, for every $t<k, n_{0}+(a+t \cdot b)$ is a member of $A$, i.e. $\operatorname{AP}_{k}\left(n_{0}+a, b\right) \subseteq A$.

The set $\mathscr{A} \mathscr{P}$ is a right ideal of $\langle\beta \mathbf{N}, \cdot\rangle$ if and only if, for any $A \in q \cdot p, A$ contains arbitrarily long arithmetic progressions; but, by the set-theoretic definition of $\cdot$, one can 
find an infinitude of $n \in \mathbf{N}$ for which $A_{n}=\{m \in \mathbf{N}: n \cdot m \in A\}$ is a member of $p$. Thus, for any $k \in \mathbf{N}$, is possible to find $a, b \in \mathbf{N}$ such that $b>0$ and $\operatorname{AP}_{k}(a, b) \subseteq A_{n}$. As such, for any $t<k$,

$$
n \cdot(a+t \cdot b)=n \cdot a+n \cdot t \cdot b
$$

is an element of $A$. Because $q$ is an ultrafilter, is possible to find an infinitude of $n_{0} \in \mathbf{N}$ for which $\mathrm{AP}_{k}\left(n \cdot a, n_{0} \cdot b\right) \subseteq A$.

Lastly, let $r \in \beta \mathbf{N} \backslash \mathscr{A} \mathscr{P}$; then, one can find a set $A$ of natural numbers that is a member of $r$ and, for some $k \in \mathbf{N}$, does not contain any arithmetic progression of length $k$. Thus, by the formula that defines $\mathscr{A} \mathscr{P}$, one can see that $\operatorname{cl}_{\beta \mathbf{N}} A$ cannot contain any ultrafilter present belonging to $\mathscr{A} \mathscr{P}$, which proves $r \in \operatorname{cl}_{\beta \mathbf{N}}(A) \subseteq \beta \mathbf{N} \backslash \mathscr{A} \mathscr{P}$. Hence, $\mathscr{A} \mathscr{P}$ is closed.

As $K(\beta \mathbf{N})$ is the minimal ideal of $\langle\beta \mathbf{N},+\rangle$, the following is true:

5.4.3 Corolary. If $\mathscr{A} \mathscr{P} \neq \emptyset$, then $K(\beta \mathbf{N},+) \subseteq \mathscr{A} \mathscr{P}$ and $K(\beta \mathbf{N}, \cdot) \subseteq \mathscr{A} \mathscr{P}$.

Let $A_{0}, \cdots, A_{r-1}$ be a non empty list of subsets of $\mathbf{N}$ that colours $\mathbf{N}$; then, for some $i<r$, it's clear that

$$
\operatorname{cl}_{\beta \mathbf{N}}\left(A_{i}\right) \cap K(\beta \mathbf{N},+) \neq \emptyset .
$$

Let $\mathcal{L}$ be the collection of all ideals of $\langle\beta \mathbf{N},+\rangle$ that are minimal; then, for any $A \subseteq A$,

$$
\operatorname{cl}_{\beta \mathbf{N}}(A) \cap K(\beta \mathbf{N},+)=\bigcup_{L \in \mathcal{L}} \operatorname{cl}_{\beta \mathbf{N}}(A) \cap L .
$$

For any set $A$ of natural numbers and any ultrafilter $p$, let

$$
A-p=\{n \in \mathbf{N}: A-n \in p\}=\{n \in \mathbf{N}: A \in n+p\} .
$$

Then, for any ultrafilter $q \in \beta \mathbf{N}, A \in p+q$ if and only if $A-p \in q$.

5.4.4 Lemma. For any set of natural numbers $A$, the following are equivalent: ${ }^{2}$

PSS1 there is a left minimal ideal $L$ of $\langle\beta \mathbf{N},+\rangle$ such that $\operatorname{cl}_{\beta \mathbf{N}}(A) \cap L \neq \emptyset$;

PSS2 there is a $p \in \beta \mathbf{N}$ for which is possible to find a finite set $F$ such that

$$
\mathbf{N}=\bigcup_{n \in F}[(A-p)-n] ; \text { and }
$$

PSS3 There is a finite set $F \subseteq \mathbf{N}$ such that

$$
\left\{\left(\bigcup_{n \in F}(A-n)\right)-m: m \in \mathbf{N}\right\} .
$$

has the FIP.

\footnotetext{
${ }^{2}$ Mutatis mutandi, this lemma can be proved for any discrete semigroup.
} 
Proof: PSS1 $\Rightarrow$ PSS2. Firstly, note that the nature of $L$ proves the equality $L=$ $\beta \mathbf{N}+q$, whenever $q$ is an element of $L$. Thus, if is possible to find a $p$ among the elements of $\operatorname{cl}_{\beta \mathbf{N}}(A) \cap L$ and a $r \in \beta \mathbf{N}$ such that $p=r+q$. Considering that

$$
p=r+q=r-\lim _{n \in \mathbf{N}}(n+q)
$$

is an element of $\operatorname{cl}_{\beta \mathbf{N}}(A)$ and such set is open in $\beta \mathbf{N}$, one can encounter a set $B \in q$ for which, given any $n \in B, n+q \in \operatorname{cl}_{\beta \mathbf{N}} A$. As such, for any element $q$ of $L$ is possible to find a $n \in \mathbf{N}$ satisfying $q \in \operatorname{cl}_{\beta \mathbf{N}}(A-n)$, which proves that the collection

$$
\left\{\operatorname{cl}_{\beta \mathbf{N}}(A-n): n \in \mathbf{N}\right\}
$$

is a cover by open subsets for $L$; as $L$ is compact, one can find a finite set $F \subseteq \mathbf{N}$ such that

$$
L \subseteq \bigcup_{n \in F} \operatorname{cl}_{\beta \mathbf{N}}(A-n)
$$

Given any $m \in \mathbf{N}$, as $L$ is a left ideal of $\langle\beta \mathbf{N},+\rangle$, one can find a $n \in F$ such that $m+p \in \operatorname{cl}_{\beta \mathbf{N}}(A-n)$. Thus, the set

$$
A-(n+m)=(A-n)-m
$$

is an element of $p$; hence, by definition, $m \in(A-p)-n$.

PSS2 $\Rightarrow$ PSS3. Let $p \in L$ and $F$ be the finite set asserting PSS2; then, for each $m \in \mathbf{N}$, one can find a $n \in F$ for which $m \in[(A-p)-n]$. Then, $[(A-n)-m] \in p$. As $p$ is an ultrafilter of $\wp(\mathbf{N})$, the result follows.

$\mathrm{PSS} 3 \Rightarrow \mathrm{PSS} 1$. Let $F$ be a finite set of $\mathbf{N}$ that testifies the validity of PSS3. As

$$
\left\{\left(\bigcup_{n \in F}(A-n)\right)-m: m \in \mathbf{N}\right\} \text {. }
$$

has the FIP, one can find an ultrafilter $q$ of $\wp(\mathbf{N})$ that contains such collection, i.e., for any $m \in \mathbf{N}$,

$$
\left(\bigcup_{n \in F}(A-n)\right)-m
$$

is an element of $q$. Thus, $m+q$ is an element of $\mathrm{cl}_{\beta \mathbf{N}}\left(\bigcup_{n \in F}(A-n)\right)$ - in particular, $q$ is an element of the last set -; being the last set closed, one has

$$
\beta \mathbf{N}+q \subseteq \operatorname{cl}_{\beta \mathbf{N}}\left(\bigcup_{n \in F}(A-n)\right)=\bigcup_{n \in F} \operatorname{cl}_{\beta \mathbf{N}}(A-n) .
$$

By the corolary 5.2.4 there is a minimal left ideal of $\langle\beta \mathbf{N},+\rangle$ contained in $\beta \mathbf{N}+q$. Given any $p \in L$, one can find a $n \in F$ such that $p \in \operatorname{cl}_{\beta \mathbf{N}}(A-n)$. Thus, $n+p \in \operatorname{cl}_{\beta \mathbf{N}}(A)$; as $L$ is a left ideal, the desired follows.

Any set $A \subseteq \mathbf{N}$ that satisfy any of the equivalent information of 5.4 .4 is called a piecewise syndetic set. A more strong condition for a set of natural numbers than PSS1 is having its closure in $\beta \mathbf{N}$ intersecting every minimal left ideal of $\langle\beta \mathbf{N},+\rangle$; sets of this type will be called syndetic.

Analogously to 5.4.4, one can prove the following: 
5.4.5 Corolary. For any set $A \subseteq \mathbf{N}$, the following assertions are equivalent

(SS1) A is syndetic;

(SS2) There is a finite $F \subseteq \mathbf{N}$ such that

$$
\mathbf{N}=\bigcup_{n \in F}(A-n) ; \text { and }
$$

(SS3) $\operatorname{cl}_{\beta \mathbf{N}}(\mathbf{N} \backslash A)$ contains no minimal left ideal;

If $A$ is piecewise syndetic, then $\operatorname{cl}_{\beta \mathbf{N}}(A)$ must contain a ultrafilter of $K(\beta \mathbf{N},+)$. The validity of 5.4 .3 proves that

5.4.6 Corolary. If $\mathscr{A} \mathscr{P} \neq \emptyset$, then every (piecewise) syndetic set contains arbitrary long arithmetic progressions.

5.4.7 Theorem. The set $\mathscr{A} \mathscr{P}$ is not empty.

Proof: For natural number $k \geq 1$, let $\triangle_{k}: \beta \mathbf{N} \rightarrow \beta \mathbf{N}^{k}$ be the diagonal map, i.e.

$$
\triangle_{k}(p)=\underbrace{\langle p, \ldots, p\rangle}_{k \text { times }}
$$

By setting

$$
\mathrm{AT}_{k}=\{\langle a, a+b, \ldots, a+(k-1) b\rangle: a, b \in \mathbf{N} \wedge b>0\},
$$

it is possible to prove that $\triangle^{-1}\left[\mathrm{cl}_{\beta \mathbf{N}^{k}} \mathrm{AT}_{k}\right]$ is constituted precisely of those ultrafilters whose members contais arithmetic progressions of length $k$. Indeed, if $p \in \triangle_{k}^{-1}\left[\mathrm{cl}_{\beta \mathbf{N}^{k}} \mathrm{AT}_{k}\right]$, then, for every $A \in p, \prod_{i<k} \operatorname{cl}_{\beta \mathbf{N}}(A)$ meets $\mathrm{AT}_{k}$, say by the pledge of some $\langle a, a+b, \ldots, a+$ $(k-1) b\rangle$. But, as

$$
\prod_{i<k} \mathrm{cl}_{\beta \mathbf{N}} A=\mathrm{cl}_{\beta \mathbf{N}^{k}}\left(\prod_{i<k} A\right)
$$

one has $\{a, a+b, \ldots, a+(k-1) b\} \subseteq A$. Contrariwise, suppose that $p$ is an ultrafilter whose elements contais arithmetic sequences of length $k$. If $A_{0}, \ldots, A_{n-1}$ is a non-empty list of elements of $p$, then, by setting $A=\bigcap_{i<n} A_{i}, U=\prod_{i<k} \operatorname{cl}_{\beta \mathbf{N}} A$ is a non-empty basic neighbourhood of $\triangle_{k}(p)$ that must intercept $\mathrm{AT}_{k}$.

Now, it is easy to see that $\mathrm{AT}_{k}$ is a subsemigroup ${ }^{3}$ of $\beta \mathbf{N}^{k}$ and $\mathrm{AT}_{k}+\triangle_{k}[\mathbf{N}] \subseteq \mathrm{AT}_{k}$. Thus, as $\mathbf{N}$ is contained in the center of $\langle\beta \mathbf{N},+\rangle$, the lemma (5.1.4) and the fact that $\triangle_{k}$ is continuous prove that

$$
\mathrm{cl}_{\beta \mathbf{N}^{k}}\left(\mathrm{AT}_{k}\right)+\mathrm{cl}_{\beta \mathbf{N}^{k}}\left(\mathrm{AT}_{k}\right) \subseteq \mathrm{cl}_{\beta \mathbf{N}^{k}}\left(\mathrm{AT}_{k}\right)
$$

and

$$
\triangle_{k}[\beta \mathbf{N}]+\operatorname{cl}_{\beta \mathbf{N}^{k}}\left(\mathrm{AT}_{k}\right) \subseteq \mathrm{cl}_{\beta \mathbf{N}^{k}}\left(\mathrm{AT}_{k}\right)
$$

\footnotetext{
${ }^{3}$ A subsemigroup of a semigroup $\langle S, *\rangle$ is a subset $S_{0}$ of $S$ such that the restriction of $*$ to $S_{0} \times S_{0}$ is a associative binary operation.
} 
Hence, by setting $S$ to be $\mathrm{cl}_{\beta \mathbf{N}^{k}}\left(\mathrm{AT}_{k}\right)$, for every $p \in \beta \mathbf{N}$,

$$
S+\triangle_{k}(p) \subseteq S \text { and } \triangle_{k}(p)+S \subseteq S
$$

Thus, being $S+\triangle_{k}(p)$ a closed left ideal of $S$ and $S$ a compact subspace of $\beta \mathbf{N}^{k}$, the Ellis-Namakura Theorem testifies the existence of a $x \in S$ such that $x+p$ is idempotente. Now, if $p \in K(\beta \mathbf{N},+)$, one can find a $y \in \beta \mathbf{N}^{k}$ such that $y+x+\triangle_{k}(p)=\triangle_{k}(p)$. Thus,

$$
\triangle_{k}(p)=x+y+\triangle_{k}(p)=x+y+\triangle_{k}(p)+y+\triangle_{k}(p)=\triangle_{k}(p)+x+\triangle_{k}(p) .
$$

As $x+\triangle_{k}(p) \in S$ and $\triangle_{k}(p)+S \subseteq S, \triangle_{k}(p) \in S$. Therefore, as stated above, $p \in \triangle^{-1}[S]$ proves that every $A \in p$ contains arbitrary long arithmetic sequences. 


\section{CHAPTER 6}

\section{The Erdős-Turán Conjecture}

\subsection{Introduction}

During this chapter, several concepts and results of measure theory is used. These concepts and results can be found in [Hal78].

The quest for properties discoursing about subsets of $\mathbf{N}$ that imply the containment of arbitrarily long arithmetical progressions will continue in this chapter. Before the formalisation, let us reflect on the heuristics. For any subset $A$ of $\mathbf{N}$, suppose that a non empty arithmetical progression $\operatorname{AP}_{k}(a, b)$ is a subset of $A$. Then,

$$
a \in A, a+b \in A, \ldots \text { and } a+(k-1) b \in A,
$$

which is equivalent to

$$
a \in A, a \in(A-b), \ldots, a \in(A-(k-1)),
$$

or

$$
a \in \bigcap_{t<k}(A-t \cdot b)
$$

Thus, $A$ contains arbitrarily long arithmetic progressions if and only if, for any natural number $k \geq 1$, one can find a positive natural number $b$ such that

$$
A \cap\left(\bigcap_{t<k}(A-t \cdot b)\right) \neq \emptyset .
$$

The trick here, which seems pedantic at first glance, is to prove that, for some good notion of measure on $\mathbf{N}$, the above set has positive measurement and thus cannot be 
empty. To this end, one has to import heuristics from measure theory; indeed, observe that if $\delta$ is a good notion of measure on $\mathbf{N}$ such that the transformation $S: \mathbf{N} \rightarrow \mathbf{N}$ given by $S(n)=n+1$ satisfies

$$
\delta(A)=\delta\left(S^{-1}[A]\right)
$$

whenever $A \subseteq \mathbf{N}$, then

$$
\delta\left(A \cap\left(\bigcap_{t<k} S^{-t b}[A]\right)\right)>0
$$

implies that $A$ contains arbitrarily long arithmetical sequences. The work that remains is to find out what is a good notion of measure that could provide such framework.

The first result of this "kind" is the Poincaré Recurrence Theorem:

6.1.1 Theorem. [Poincaré - 1890; Carathéodory - 1919] Let $\langle X, \mathcal{B}, \mu\rangle$ be a probabilistic space and $T: X \rightarrow X$ a measure preserving map - i.e., for any $B \in \mathcal{B}, T^{-1}[B] \in \mathscr{B}$ and $\mu(B)=\mu\left(T^{-1}[B]\right)$ - Then, for each $A \in \mathscr{B}$ such that $\mu(A)>0$, there exists a positive $n \in \mathbf{N}$ such that

$$
\mu\left(A \cap T^{-n}[A]\right)>0 .
$$

The process of iteration on the above theorem produces quite interesting results to number theory. Indeed, let $\langle X, \mathcal{B}, \mu\rangle$ be a probabilistic space and $T: X \rightarrow X$ a measure preserving map; given a $A \in \mathscr{B}$ positive valued under $\mu$, one can find a natural number $n_{0}>1$ such that

$$
\mu\left(A \cap T^{-n_{0}}[A]\right)>0 ;
$$

using the information provided by the theorem for $A \cap T^{-n_{0}}[A]$ instead of $A$, one can find a positive natural $n_{1}$ such that

$$
\mu\left(A \cap T^{-n_{0}}[A] \cap T^{-n_{1}}\left[A \cap T^{-n_{0}}[A]\right]\right)>0 .
$$

It's easy to see that

$$
A \cap T^{-n_{0}}[A] \cap T^{-n_{1}}\left[A \cap T^{-n_{0}}[A]\right]=A \cap T^{-n_{0}}[A] \cap T^{-n_{1}}[A] \cap T^{-\left(n_{0}+n_{1}\right)}[A] .
$$

For any $G \subseteq \mathbf{N}$, let

$$
\operatorname{FS}(G)=\left\{\sum_{n \in F} n: F \in[G]^{<\omega} \backslash\{\emptyset\}\right\}
$$

be the set of all finite sums of elements of $G$. Then, iterating the Poincaré's Recurrence Theorem, for any positive $\mu$-mensurable set $A$ and any $k \geq 1$, one can find a finite set of natural numbers $F$ such that $|F|=k$ and

$$
\mu\left(A \cap \bigcap_{x \in F S(F)} T^{-x}[A]\right)>0 .
$$

Thus, Poincaré Recurrence is a optimal tool to deal with finite sums of a given set of natural numbers (or, in a more abstract setting, a semigroup). But, to use Poincaré's recurrence theorem, is necessary to transfer number theoretical situations to a measure theoretical ones. 


\subsection{The Measure Problem}

Note that there can be no $\mu: \wp(\mathbf{N}) \rightarrow[0,1]$ that is monotonic, shift invariant and countably additive function such that $\mu(\mathbf{N})=1$. Indeed, for any $m, n \in \mathbf{N}$, as $\mu$ is shift invariant, $\mu(\{n\})=\mu(\{m\})$; moreover, either $\mu(\{n\})=0$ or $\mu(\{n\})>0$. Both cases leads to an absurd because as the countable additivity of $\mu$ proves that

$$
\mu(\mathbf{N})=\sum_{n \geq 0} \mu(\{n\})
$$

Following [LT15], any monotonic finitely subadditive function $\delta: \wp(\mathbf{N}) \rightarrow[0,1]$ satisfying

(DF1) $\delta(\mathbf{N})=1$

(DF2) if $n \in \mathbf{N}$ is not zero, $\delta(n \cdot A)=\frac{1}{n} \delta(A)$; and

(DF3) given any $n \in \mathbf{N}, \delta(n+A)=\delta(A)$.

is called upper density function of $\mathbf{N}$. It is easy to see that, if $A$ is a finite set of natural numbers and $\delta$ is a upper density function of $\mathbf{N}$, then $\delta(A)=0$. Indeed, if $k \geq 1$ is a natural number, then

$$
\delta(\{1\})=\delta(\{1\}+k-1)=\delta(\{k\})=\frac{1}{k} \delta(\{1\})
$$

Thus, $\delta(\{n\})=0$, for every $n \in \mathbf{N}$; also

$$
\delta(\emptyset)=\delta(k \emptyset)=\frac{1}{k} \delta(\emptyset),
$$

which proves that $\delta(\emptyset)=0$. Finally, if $F \subseteq \mathbf{N}$ is finite and non-empty,

$$
0 \leq \delta(F)=\delta\left(\bigcup_{n \in F}\{n\}\right) \leq \sum_{n \in F} \delta(\{n\})=0 .
$$

For any $A \subseteq \mathbf{N}$ and $n \in \mathbf{N}$, let

$$
A / n=\{m \in \mathbf{N}: n \cdot m \in A\} .
$$

6.2.1 Lemma. If $\delta$ is an upper density function of $\mathbf{N}$, then, for every $A \subseteq \mathbf{N}$ and a natural number $n \geq 0$,

$$
\delta(A-n)=\delta(A)
$$

If $n \geq 1$, then

$$
\delta(A / n)=n \delta(A)
$$


Proof: For any $n \in \mathbf{N}$, the set

$$
A \backslash[(A-n)+n]
$$

is finite. Thus, as $\delta$ is finitely subadditive, it's observable that

$$
0=\delta(A \backslash[(A-n)+n])=\delta(A)-\delta([(A-n)+n])=\delta(A)-\delta(A-n),
$$

which proves that $\delta(A)=\delta(A-n)$.

If $n \geq 1$, then the set

$$
A \backslash(n \cdot A / n)
$$

is a finite set. Thus,

$$
0=\delta(A \backslash(n \cdot A / n))=\delta(A)-\delta(n \cdot A / n)=\delta(A)-\frac{1}{n} \delta(A / n),
$$

which settles the desired.

The next result, merely a reformulation of 3.9.1 for the Boolean algebra $\wp(\mathbf{N})$, will help to translate properties concerning subsets of $\mathbf{N}$ to topological properties of $\beta \mathbf{N}$.

6.2.2 Corolary. For any predicate $\varphi$ discoursing about subsets of $\mathbf{N}$, let

$$
\mathscr{R}_{\varphi}=\{p \in \beta \mathbf{N}: \forall A \in p(\varphi(A))\} .
$$

Then the following are equivalent:

a) If $\varphi(A)$, then $\operatorname{cl}_{\beta \mathbf{N}}(A) \cap \mathscr{R}_{\varphi} \neq \emptyset$; and

b) (i) $\neg \varphi(\emptyset)$;

(ii) if $A \subseteq B \subseteq \mathbf{N}$, then $\varphi(A) \rightarrow \varphi(B)$; and

(iii) if $A, B \subseteq \mathbf{N}$, then $\varphi(A \cup B) \rightarrow \varphi(A) \vee \varphi(B)$.

Moreover, the set $\mathscr{R}_{\varphi}$ is closed in $\beta \mathbf{N}$.

6.2.3 Corolary. For any predicates $\varphi$ and $\psi$ discoursing about subsets of $\mathbf{N}$ such that $\varphi$ satisfy one of the equivalent assertions of 6.2 .2 , the following are equivalent:

(i) For any set $A \subseteq \mathbf{N}, \varphi(A) \rightarrow \psi(A)$; and

(ii) $\mathscr{R}_{\varphi} \subseteq \mathscr{R}_{\psi}$.

6.2.4 Lemma. Let $\delta$ be a upper density function of $\mathbf{N}$ and

$$
\mathscr{D}_{\delta}=\{p \in \beta \mathbf{N}: \forall A \in p(\delta(A)>0)\} .
$$

Then, $\mathscr{D}_{\delta}$ is a closed ideal of $\langle\beta \mathbf{N},+\rangle$ and an left ideal of $\langle\beta N, \cdot\rangle$. 
Proof: Clearly the statement "having upper density greater than 0" "satisfies (i),(ii) and (iii) of 6.2 .2 , and thus $\mathscr{D}_{\delta}$ is a closed subset of $\beta \mathbf{N}$. Now, given any $p \in \mathscr{D}_{\delta}$ and $q \in \beta \mathbf{N}$, a set $A$ is an element of $p+q$ if and only if the set of all $n \in \mathbf{N}$ such that $A-n \in p$ is a member of $q$; thus, selecting a $n \in \mathbf{N}$ such that $A-n \in p$,

$$
\delta(A)=\delta(A-n)>0
$$

which proves that $q+p \in \mathscr{D}_{\delta}$. Now, for any $n \in \mathbf{N}$ and $A \subseteq \mathbf{N}, \delta(A)>0$ implies that $\delta(n+A)>0$ (and vice versa). Consequently, $\mathbf{N}+\mathscr{D}_{\delta} \subseteq \mathscr{D}_{\delta}$ for, given a $p \in \mathscr{D}_{\delta}, A \in n+p$ if and only if $n+A$ is an element of $p$. Hence, for any $q \in \beta \mathbf{N}$, the fact that $\mathscr{D}_{\delta}$ is a closed subset of $\beta \mathbf{N}$ proves that

$$
q+p=q-\lim _{n \in \mathbf{N}}(n+p) \in \mathscr{D}_{\delta}
$$

An analogous use of 6.2 .1 proves that $\mathscr{D}_{\delta}$ is also a left ideal of $\langle\beta \mathbf{N}, \cdot\rangle$.

If $S^{\uparrow}$ is the set of all increasing sequences of natural numbers, define (as in [Hin88])

$$
\mathscr{P}(A)=\left\{r \in \mathbf{R}: \exists s, t \in S^{\uparrow} \forall n \in \mathbf{N}\left(s_{n} \cdot r \leq\left|A \cap\left[t_{n}+1, t_{n}+s_{n}\right]\right|\right)\right\}
$$

Evidently, $\mathscr{P}(A) \subseteq \mathbf{I}$ and it's not empty. Then, the Banach upper density of $A$, namely $\mathfrak{b}(A)$, is the supremum of $\mathscr{P}(A)$. The more alerted reader should get confused at this point, for the definition given above is not the usual one; the equivalence between the two definitions, as well as two more equivalent definitions, will be given below.

6.2.5 Lemma. [Hin88] For each $A \subseteq \mathbf{N}$, let

$$
b=\sup \left\{r \in \mathbf{I}: \exists t \in S^{\uparrow} \forall n \in \mathbf{N} \forall k \leq n\left(r \cdot k \leq\left|A \cap\left[t_{n}+1, t_{n}+k\right]\right|\right)\right\} .
$$

Then, $\mathfrak{b}(A)=b$.

Proof: Evidently, by testimony of the increasing sequence $\langle n\rangle, b \leq \mathfrak{b}(A)$. If $\boldsymbol{b}(A)>$ $b$, then $\varepsilon=b(A)-b>0$. Thus, as $\mathfrak{b}(A)>b+2 \varepsilon / 3$ and using the definition of the Banach upper density, one can find increasing sequences $\left\langle t_{n}\right\rangle$ and $\left\langle s_{n}\right\rangle$ such that, for each $n \in \mathbf{N}$,

$$
\left|A \cap\left[t_{n}+1, t_{n}+s_{n}\right]\right| \geq s_{n} \cdot\left(b+\frac{2 \varepsilon}{3}\right) .
$$

As $b<b+\varepsilon / 3$, there is an increasing sequence of natural numbers $\left\langle x_{n}\right\rangle$ such that, for any $n \in \mathbf{N}$,

$$
\left|A \cap\left[x_{n}+1, x_{n}+k\right]\right|<k\left(b+\frac{\varepsilon}{3}\right),
$$

whenever $k \leq n$. Thus, one can find natural numbers $m$ and $n$ such that, for each $z \geq m$ and each $v \leq n$,

$$
|A \cap[z+1, z+v]|<v\left(b+\frac{\varepsilon}{3}\right) .
$$

Let $k \in \mathbf{N}$ such that $t_{k} \geq m$ and $s_{k} \geq 3 n / \varepsilon$. Suppose that are two non empty list of natural numbers $u_{1}, \ldots, u_{l}$ and $v_{1}, \ldots, v_{l}$ satisfying 
(1) $u_{1}=t_{k}$ and $t_{k}+s_{k}-n \leq u_{l-1}+v_{l-1} \leq t_{k}+s_{k}$;

(2) if $i \leq l, v_{i} \leq n$ and $\left|A \cap\left[u_{i}+1, u_{i}+v_{i}\right]\right|<v_{i}(b+\varepsilon / 3)$; and

(3) if $i<l, u_{i+1}=u_{i}+v_{i}$.

Then, using (3) and (1),

$$
\sum_{i=1}^{l} v_{i}=\left(\sum_{i<l} v_{i}\right)+v_{l}=\left(\sum_{i<l} u_{i+1}-u_{i}\right)+v_{l}=u_{l}+v_{l}-t_{k} \leq s_{k} .
$$

And using (2) and (1) and the fact that $s_{k} \geq 3 n / \varepsilon$,

$$
\begin{aligned}
\left|A \cap\left[t_{k}+1, t_{k}+s_{k}\right]\right| & =\left|A \cap\left[t_{k}+1, u_{l}+v_{l}\right]\right|+\left|A \cap\left[u_{l}+v_{l}+1, t_{k}+s_{k}\right]\right| \leq \\
& \leq \sum_{i=1}^{l}\left|A \cap\left[u_{i}, u_{i}+v_{i}\right]\right|+n<\sum_{i=1}^{l} v_{i}\left(b+\frac{\varepsilon}{3}\right)+n \leq \\
& \leq s_{k}\left(b+\frac{\varepsilon}{3}\right)+n \leq s_{k}\left(b+\frac{2 \varepsilon}{3}\right) .
\end{aligned}
$$

which is absurd. Thus, $\mathfrak{b}(A) \leq b$.

As the definition goes, for any $k \geq 1$, if $\mathcal{L}_{k}$ is the set of all intervals inside $\mathbf{N}$ whose length is not smaller than $k$, one can prove that

$$
b(A)=\sup \bigcap_{k \geq 1} \bigcup_{I \in \mathcal{L}_{k}}\left\{r \in \mathbf{I}: r \leq \frac{|A \cap I|}{|I|}\right\} .
$$

6.2.6 Lemma. Let $A$ be a set of natural numbers whose Banach upper density is positive. Then there is a sequence $\left\langle I_{n}\right\rangle$ of intervals of $\mathbf{N}$ whose sequence of cardinalities diverges and

$$
\lim _{n \rightarrow \infty} \frac{\left|A \cap I_{n}\right|}{\left|I_{n}\right|}=b(A)
$$

The sequence $\left\langle I_{n}\right\rangle$ above is called Følner sequence. The following equation is the usual definition of the Banach density:

6.2.7 Lemma. [Son78; Ten15] For any $A \subseteq \mathbf{N}$

$$
\mathfrak{b}(A)=\lim _{n \geq 1} \max _{m \geq 0} \frac{|A \cap[m+1, m+n]|}{n} .
$$

6.2.8 Example. Let $A$ be a syndetic set. Then, $b(A)>0$. Indeed, if $A \subseteq \mathbf{N}$ is a syndetic set of $\mathbf{N}$, then one can find a sequence of natural numbers $\left\langle t_{n}\right\rangle$ and an increasing sequence of natural numbers $\left\langle s_{n}\right\rangle$ such that

$$
\left\{t_{n}+s_{m}: m, n \in \mathbf{N} \wedge m \leq n\right\} \subseteq A,
$$

Thus, for each $n \in \mathbf{N}$,

$$
0<\left|A \cap\left\{t_{n}+s_{m}: m \leq n\right\}\right| \leq m \leq n \leq s_{n},
$$

as $\left\langle s_{n}\right\rangle$ is increasing. 
In [ET36], Erdős and Turán posed the question if $A \subseteq \mathbf{N}$ is a set having positive Banach upper density, is it true that $A$ contains arbitrarily long arithmetic progression? The answer for this question is affirmative and was proved combinatorially by Endre Szemerédi in [Sze75]. In the present account, a prove of this fact will be presented in the style of ergodic theory, formulated by Hillel Furstenberg in [Fur77; FKO82], with a topological emphasis as can be seen in [HS12].

In fact, the original question was formulated in terms of the natural upper density ${ }^{1}$. If $A$ is a set of natural numbers, then its natural upper density is given by

$$
\bar{d}(A)=\limsup _{n \rightarrow \infty} \frac{|A \cap[1, n]|}{n}
$$

The same arguments used to prove 6.2 .1 can be used to prove the following:

6.2.9 Lemma. The Banach upper density and the natural upper density are, as the very name suggest, upper densities of $\mathbf{N}$.

6.2.10 Lemma. Let $\mathcal{B}$ be a $\sigma$-algebra on $X$ and $\mu: \mathcal{B} \rightarrow \mathbf{I}$ be a finitely additive function whose value on $X$ is 1 . If $\left\langle A_{n}\right\rangle$ is a $\subseteq$-decreasing sequence of subsets within $X$ such that $\bigcap_{n \in \mathbf{N}} A_{n}=\emptyset$ and $\lim _{n \rightarrow \infty} \mu\left(A_{n}\right)=0$, then $\mu$ is countably additive.

Proof: Consider $A=\bigcup_{n \in \mathbf{N}} A_{n}$ and, for each natural number $m, B_{m}=\bigcup_{k \leq m} A_{k}$. Obviously, $A=\bigcup_{m \in \mathbf{N}} B_{m}$. As $\mu$ is finitely additive, for every $m \in \mathbf{N}$,

$$
\mu(A)=\mu\left(B_{m} \cup\left(A \backslash B_{m}\right)\right)=\mu\left(B_{m}\right)+\mu\left(A \backslash B_{m}\right) .
$$

But the sequence $A \backslash B_{m}$ converges to $\emptyset$, thus

$$
\begin{aligned}
\mu(A) & =\mu\left(B_{m} \cup\left(A \backslash B_{m}\right)\right)=\lim _{m \rightarrow \infty} \mu\left(B_{m}\right)+\lim _{m \rightarrow \infty} \mu\left(A \backslash B_{m}\right)= \\
& =\lim _{m \rightarrow \infty} \sum_{n \leq m} \mu\left(A_{n}\right)=\sum_{n \geq 0} \mu\left(A_{n}\right) .
\end{aligned}
$$

The following result is a consequence of the Caratheodory Extension Theorem for outer measures (see, for instance, [Hal78]):

6.2.11 Lemma. Let $\mathcal{A}$ be a Boolean algebra of subsets of some set $X$. If $\mu$ is a countably additive measure defined in $\mathcal{A}$, then there is a $\sigma$-algebra $\mathscr{B}$ of subsets of $X$ such that function $\bar{\mu}: \mathscr{B} \rightarrow[0, \infty]$ given by

$$
\bar{\mu}(A)=\inf \left\{\sum_{n \geq 0} \mu\left(A_{n}\right): \forall n \in \mathbf{N}\left(A_{n} \in \mathcal{A} \wedge A \subseteq \bigcup_{n \in \mathbf{N}} A_{n}\right)\right\}
$$

is an countably additive measure on $\mathcal{B}$ that extends $\mu$ and every element of $\mathcal{A}$ is $\mathcal{B}$ measurable.

\footnotetext{
${ }^{1}$ The equivalence of this two statement is proved in lemma 6.4.3
} 
6.2.12 Theorem. [FKO82] If $A$ is a set of natural numbers whose Banach density is positive, then there is a probability space $\langle X, \mathscr{B}, \mu\rangle$, a $\mu$-measurable $A_{0}$ and a measure preserving map $T: X \rightarrow X$ such that

(FR1) $X$ is a compact space and $T: X \rightarrow X$ is a homeomorphism;

(FR2) $\mu\left(A_{0}\right)=\mathfrak{b}(A)$; and

(FR3) for every finite set $F \subseteq \mathbf{N}$,

$$
\mu\left(A_{0} \cap \bigcap_{m \in F} T^{-m}\left[A_{0}\right]\right) \leq \mathfrak{b}^{*}\left(A \cap \bigcap_{m \in F}(A-m)\right)
$$

Proof: $\operatorname{As~}^{*}(A)>0$, there is a Følner sequence $\left\langle F_{n}\right\rangle$ such that $\lim _{n \rightarrow \infty}\left|F_{n}\right|=\infty$ and

$$
\mathfrak{b}^{*}(A)=\lim _{n \rightarrow \infty} \frac{\left|A \cap F_{n}\right|}{\left|F_{n}\right|} .
$$

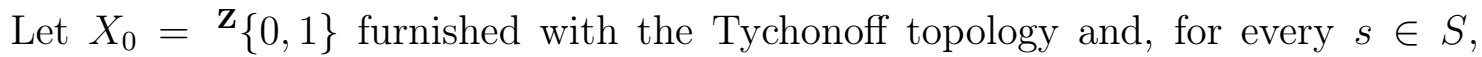
let $T_{s}: X_{0} \rightarrow X_{0}$ given by $T_{s}(x)(n)=x(n+1)$. The cancellative property of the sum implies that $T_{s}$ is a homeomorphism. If $\chi_{A}$ is the characteristic function of $A$, let $X=\operatorname{cl}_{X_{0}}\left\{T_{s}\left(\chi_{A}\right): s \in S\right\}$. Evidently, $X$ is a compact space and $T_{s}$ is a homeomorphism of $X$ into itself.

If $n \in \mathbf{N}$, consider the open and closed subsets of $X$ given by $D_{n}=X \cap \mathrm{p}_{n}^{-1}[\{1\}]$. Let $\mathcal{A}$ be the smallest Boolean algebra generated by $\left\{D_{n}: n \in \mathbf{N}\right\}$ and $\mathscr{B}$ the smallest $\sigma$-algebra generated by $\left\{D_{n}: n \in \mathbf{N}\right\}$. Now, for every $n \in \mathbf{N}, T_{s}\left[D_{n+1}\right]=D_{n}$ and $T_{s}^{-1}\left[D_{n}\right]=D_{n+1}$. Hence, $\mathscr{B}$ is closed under application of $T_{s}$ and $T_{s}^{-1}$.

Let $\varphi: \wp(X) \rightarrow \wp(\mathbf{N})$ given by $\varphi(B)=\left\{n \in \mathbf{N}: T_{s}^{n}\left(\chi_{A}\right) \in B\right\}$. For any $B, C \subseteq X$ it's easy to see that $\varphi(B \cap C)=\varphi(B) \cap \varphi(C)$; moreover, if $B \cap C=\emptyset$, then $\varphi(B \cup C)=$ $\varphi(B) \cup \varphi(C)$. Thus, given any ultrafilter $p \in \beta \mathbf{N}$, the function $\nu: \wp(X) \rightarrow[0,1]$ defined by

$$
\nu(B)=p-\lim \frac{\left|\varphi(B) \cap I_{n}\right|}{\left|I_{n}\right|}
$$

is finitely additive monotonic and assumes 1 when evaluated on $X$. Moreover, if $B \subseteq X$, then

$$
\varphi\left(T_{s}^{-1}[B]\right)=(-1+\varphi(B)) \cap \mathbf{N}
$$

Thus,

$$
\nu\left(T_{s}^{-1}[B]\right)=p-\lim \frac{\left|\varphi\left(T^{-1}[B]\right) \cap I_{n}\right|}{\left|I_{n}\right|}=p-\lim \lim _{n \in \mathbf{N}} \frac{\left|\varphi(B) \cap I_{n}\right|}{\left|I_{n}\right|}=\nu(B),
$$

which makes $T_{s} \nu$-invariant.

As $\mathcal{A}$ is composed by open-and-closed subsets of $X$, if $\left\langle B_{n}\right\rangle$ is a $\subseteq$-decreasing sequence inside $\mathcal{A}$ such that $\bigcap_{n \in \mathbf{N}} A_{n}=\emptyset$, then the compactness of $X$ testifies the existence of some $m \in \mathbf{N}$ such that $A_{n}=\emptyset$ whenever $n \geq m$. In this case, $\lim _{n \rightarrow \infty} \nu\left(A_{n}\right)=$ 
0 and thus, by $6.2 .10, \nu$ is countably additive in $\mathcal{A}$. Hence, with the help of 6.2 .11 , there is a $\sigma$-algebra $C$ of subsets of $X$ such that

$$
\mu(B)=\inf \left\{\sum_{n \geq 0} \nu\left(B_{n}\right): \forall n \in \mathbf{N}\left(B_{n} \in \mathcal{A} \wedge B \subseteq \bigcup_{n \in \mathbf{N}} B_{n}\right)\right\}
$$

is a measure on $\langle X, C\rangle$ that extends $\nu$ and $\mathcal{A} \subseteq C$. As $\mathcal{A} \subseteq \mathcal{B}$, is evidently that $\mathscr{B} \subseteq C$. Thus, $\langle X, \mathscr{B}, \mu\rangle$ is a probability space.

Now, if $B \in \mathscr{B}$, then for any natural $n$, as $T$ is an automorphism of $X$,

$$
\begin{aligned}
\mu(B) & =\inf \left\{\sum_{C \in \mathcal{A}_{0}} \nu(C): \mathcal{A}_{0} \in[\mathcal{A}]^{\leq \omega} \wedge B \subseteq \bigcup \mathcal{A}_{0}\right\}= \\
& =\inf \left\{\sum_{C \in \mathcal{A}_{0}} \nu\left(T^{-1}[C]\right): \mathcal{A}_{0} \in[\mathcal{A}]^{\leq \omega} \wedge T^{-1}[C] \subseteq \bigcup_{C \in \mathcal{A}_{0}} T^{-1}[C]\right\}= \\
& =\mu\left(T^{-1}[B]\right) .
\end{aligned}
$$

Being $A_{0}=D_{0}$,

$$
\begin{aligned}
\varphi\left(A_{0}\right) & =\left\{n \in \mathbf{N}: T^{n}\left(\chi_{A}\right) \in D_{0}\right\}=\left\{n \in \mathbf{N}: T^{n}\left(\chi_{A}\right)(0)=1\right\}= \\
& =\left\{n \in \mathbf{N}: \chi_{A}(n)=1\right\}=A
\end{aligned}
$$

and

$$
\mu\left(A_{0}\right)=\nu\left(A_{0}\right)=p-\lim \frac{\left|\varphi\left(A_{0}\right) \cap I_{n}\right|}{\left|I_{n}\right|}=p-\lim \frac{\left|A \cap I_{n}\right|}{\left|I_{n}\right|}=\mathfrak{b}(A) .
$$

Finally, if $F$ is a finite subset of $\mathbf{N}$, as

$$
\mu\left(A_{0} \cap \bigcap_{n \in F} T^{-n}\left[A_{0}\right]\right)=p-\lim _{k \in \mathbf{N}} \frac{\left|A \cap \bigcap_{n \in \mathbf{N}}(A[n]) \cap I_{k}\right|}{\left|I_{k}\right|}
$$

and, for each $k \in \mathbf{N}$ and being $\mathcal{L}_{k}$ the collection of all intervals of $\mathbf{N}$ whose length is greater or equal to $k$,

$$
\frac{\left|A \cap\left(\bigcap_{n \in F} A[n]\right) \cap I_{k}\right|}{\left|I_{k}\right|} \leq \sup \bigcup_{m \in \mathbf{N}} \bigcap_{I \in \mathcal{L}_{m}}\left\{r \in \mathbf{I}: \frac{|A \cap I|}{|I|} \geq r\right\},
$$

one has

$$
\mu\left(A_{0} \cap \bigcap_{n \in F} T^{-n}\left[A_{0}\right]\right) \leq \mathfrak{b}\left(A \cap \bigcap_{n \in F} A[n]\right)
$$

as required.

Now, let $A \subseteq \mathbf{N}$ be a set of positive Banach density. Then, one can find a probability space $\langle X, \mathcal{B}, \mu\rangle$, a measure preserving map $T: X \rightarrow X$ and a set $A^{\prime} \in \mathscr{B}$ such that, for any finite $F \subseteq \mathbf{N}$,

$$
\mu\left(A^{\prime} \cap \bigcap_{m \in F} T^{-m}\left[A^{\prime}\right]\right) \leq \mathfrak{b}^{*}\left(A \cap \bigcap_{m \in F}\{n \in \mathbf{N}: n+m \in A\}\right) .
$$


For any $k \geq 1$, if one can find a $b \in \mathbf{N}$ such that

$$
\mu\left(\bigcap_{i<k} T^{-i b}\left[A^{\prime}\right]\right)>0
$$

then, as $F=\{0, b, \ldots,(k-1) b\}$ is finite,

$$
0<b^{*}\left(A \cap \bigcap_{m \in F}\{n \in \mathbf{N}: n+m \in A\}\right) .
$$

Thus, by choosing any

$$
a \in A \cap \bigcap_{m \in F}\{n \in \mathbf{N}: n+m \in A\}
$$

one has $\operatorname{AP}_{k}(a, b) \subseteq A$.

The only thing remaining to do now is to find a recurrence result, in the fashion of (6.1.1), that could grantees the non-voidness of the above intersection.

6.2.13 Theorem. [Fur77] Let $\langle X, \mathscr{B}, \mu\rangle$ be a probability space and $T: X \rightarrow X$ be a measure preserving map. Then, for any set $B \in \mathscr{B}$ of positive measure and any $k \geq 1$, is possible to find a $b \in \mathbf{N}$ such that

$$
\mu\left(\bigcap_{m<k} T^{-m b}[B]\right)>0 .
$$

The proof of 6.2.13 requires a long development of ergodic theory which escapes the proposal of this dissertation. A didactic exposition of this result, alongside with its number theoretical and combinatorial consequences, can be found in [Fur81] and [MAM07].

6.2.14 Corolary. [Sze75; Fur77](Szemerédi Theorem) Every set of natural numbers whose Banach density is positive has arbitrarily long arithmetic sequences.

\subsection{Equivalences of the Erdôs-Turán Conjecture}

Consider a set $A$ composed by positive natural numbers whose natural upper density is positive, say $2 \alpha$ for some $\alpha \in \mathbf{I}$. Then there is a sequence of positive naturals $\left\langle n_{k}\right\rangle$ such that

$$
\lim _{k \rightarrow \infty} \frac{\left|A \cap\left[1, n_{k}\right]\right|}{n_{k}}=2 \alpha
$$

and, for any $k \in \mathbf{N}$,

$$
\left|A \cap\left[1, n_{k}\right]\right| \geq \alpha n_{k} \quad \text { and } \quad n_{k+1} \geq \frac{2 n_{k}}{\alpha} .
$$

Thus, for every $k \in \mathbf{N}$,

$$
\mid A \cap\left[n_{k}, n_{k+1}\left[\mid \geq \alpha n_{k+1}-n_{k} \geq \alpha n_{k+1}-\frac{\alpha n_{k+1}}{2}=\frac{\alpha n_{k+1}}{2} .\right.\right.
$$


Therefore,

$$
\sum_{n \in A} \frac{1}{n} \geq \sum_{k \geq 0} \sum_{n \in A \cap\left[n_{k}, n_{k+1}[\right.} \frac{1}{n} \geq \sum_{k \geq 0} \frac{\alpha n_{k+1}}{2 n_{k+1}}=\sum_{k \geq 0} \frac{\alpha}{2},
$$

which proves that the sum of reciprocals of all elements within $A$ must diverge. After the solution of 6.2.14 was given by Endre Szemerédi, in a talk in honour to Paul Turán, Erdôs conjectured:

6.3.1 Conjecture. (Erdős-Turán Conjecture) Being $A$ a set of positive natural numbers whose sum of reciprocals diverges, does $A$ contain arbitrarily long arithmetic progressions?

Even the weaker version of 6.3.1 in which the question is restricted to arithmetic progressions of length 3 is, by the moment when this treatise is being written, open. In this section, some combinatorial equivalences of 6.3.1, due to Erdôs, Gerver and Hindman, are presented.

Remember that

$$
\mathscr{A} \mathscr{P}=\left\{p \in \beta \mathbf{N}: \forall A \in p \forall k \in \mathbf{N} \exists a, b \in \mathbf{N}\left(\operatorname{AP}_{k}(a, b) \subseteq A\right)\right\}
$$

and consider the following sets

$$
\mathscr{D}=\{p \in \beta \mathbf{N}: \forall A \in p(\overline{\mathrm{d}}(A)>0)\} \quad \text { and } \quad \mathscr{B}=\{p \in \beta \mathbf{N}: \forall A \in p(\mathcal{b}(A)>0)\} .
$$

Then, Szemerédi Theorem is equivalent to the fact that $\mathscr{B} \subseteq \mathscr{A} \mathscr{P}$ (indeed, it will be proved that $\mathscr{B} \subseteq \mathscr{A} \mathscr{P}$ and $\mathscr{D} \subseteq \mathscr{A} \mathscr{P}$ are equivalent assertions).

6.3.2 Lemma. $\mathscr{B}=\operatorname{cl}_{\beta \mathbf{N}}\left(\mathbf{N}^{*}+\mathscr{D}\right)$.

Proof: For every subset $A$ of $\mathbf{N}, \mathfrak{b}(A) \geq \bar{d}(A)$, thus $\mathscr{D} \subseteq \mathscr{B}$. As $\mathscr{B}$ is a left closed ideal of $\langle\beta \mathbf{N},+\rangle$,

$$
\mathbf{N}^{*}+\mathscr{D} \subseteq \mathbf{N}^{*}+\mathscr{B} \subseteq \mathscr{B},
$$

which implies that $\operatorname{cl}_{\beta \mathbf{N}}\left(\mathbf{N}^{*}+\mathscr{D}\right) \subseteq \mathscr{B}$.

Conversely, given any $p \in \mathscr{B}, A \in p$ and $\alpha>0$ such that $b^{*}(A)>\alpha$, using 6.2.5 one can find an increasing sequence $\left\langle t_{n}\right\rangle$ such that, for every $n \in \mathbf{N}$ and every natural $k \leq n$,

$$
\left|A \cap\left\{t_{n}+1, \ldots, t_{n}+k\right\}\right| \geq k \alpha .
$$

Thus, for each $m \in \mathbf{N}$, let $T_{m}=\left\{n \in \mathbf{N}: n \geq m \wedge t_{n}+m \in A\right\}$. Define $S_{0}=\mathbf{N}$ and, for each $m \in \mathbf{N}$

$$
S_{m+1}= \begin{cases}T_{m+1} \cap S_{m} & , \text { if }\left|T_{m+1} \cap S_{m}\right|<\mathbf{N} ; \text { or } \\ S_{m} \backslash T_{m+1} & , \text { otherwise. }\end{cases}
$$

In this case, for each $m \in \mathbf{N}, S_{m}$ is infinite. Moreover, being

$$
B=\left\{n \in \mathbf{N}: S_{n+1}=S_{n} \cap T_{n+1}\right\},
$$

it follows that $\bar{d}(B)>0$. Indeed, for each $k \in \mathbf{N}$ and $n \in S_{k}$ such that $n \geq k$, select a natural number $m$ from $[1, k]$ so that $t_{n}+m \in A$; if $m \notin B$, then $n \in S_{k} \subseteq S_{m}=S_{m-1} \backslash T_{m}$ and therefore $t_{n}+m \notin A$. Thus, $m \in B$ and

$$
|B \cap\{0, \ldots, k\}| \geq\left|A \cap\left\{t_{n}+1, \ldots, t_{n}+k\right\}\right| \geq k \alpha .
$$


Now, as $B$ has positive upper natural density, let $r \in \mathscr{D}$ such that $B \in r$. For each $m \in \mathbf{N}$, let $U_{m}=\left\{t_{n}: n \in S_{m}\right\}$ and, as these sets are infinite and has the FIP, $q \in \mathbf{N}^{*}$ such that $\left\{U_{m}: m \in \mathbf{N}\right\} \subseteq q$. For every $m \in B$, one has

$$
\left\{t_{n}: n \in S_{m}\right\} \subseteq\{n \in \mathbf{N}: n+m \in A\}
$$

and $q$ extends the sets $U_{m}$, from which one deduces $A \in q+r$. Thus, $\operatorname{cl}_{\beta \mathbf{N}}(A)$ is a neighbourhood of $p$ that meets $\mathbf{N}^{*}+\mathscr{D}$.

Let

$$
\mathscr{E}=\left\{p \in \beta \mathbf{N}: \forall A\left(A \in p \wedge 0 \notin A \rightarrow \sum_{n \in A} \frac{1}{n}=\infty\right)\right\}
$$

Then the Erdôs-Turán conjecture can be reformulated as $\mathscr{E} \subseteq \mathscr{A} \mathscr{P}$.

6.3.3 Lemma. $\mathscr{E}$ is a right ideal of both $\langle\beta \mathbf{N},+\rangle$ and $\langle\beta \mathbf{N}, \cdot\rangle$.

Proof: Let $p \in \mathscr{E}$ and $q \in \beta \mathbf{N}$. Given any $A \in p+q$ whose elements are positive, the set of all $n \in \mathbf{N}$ such that $A-n \in p$ is a member of $q$. Thus, as $A-n=\{m-n$ : $m \in A \wedge m \geq n\}$, the limit

$$
\sum_{m \in A \wedge m>n} \frac{1}{m-n}
$$

is divergent; this information culminates in the divergence of $\sum_{m \in A} \frac{1}{m}$, which settles $p+q \in \mathscr{E}$. If $A \in p \cdot q$, choose some $n \geq 1$ such that $A / n \in p$. Then, the set $A \backslash(n \cdot A / n)$ is finite; consequently, one has the following equality between limits

$$
\sum_{m \in A} \frac{1}{m} \geq \sum_{m \in A / n} \frac{1}{m n}=\frac{1}{n} \sum_{m \in A / n} \frac{1}{m}
$$

which proves that sum of reciprocals composed by elements from $A$ is divergent.

Let $\mathscr{S}_{0}$ be the set of all ultrafilters $p$ of $\wp(\mathbf{N})$ such that, given any $A \in p$, one can find increasing sequences $\left\langle s_{n}\right\rangle$ and $\left\langle t_{n}\right\rangle$ of natural numbers satisfying $0 \notin\left\{s_{n}: n \in \mathbf{N}\right\}$,

$$
\left\{t_{n}+s_{m}: m, n \in \mathbf{N} \wedge m \leq n\right\} \subseteq A \text { and } \quad \sum_{n \geq 0} \frac{1}{s_{n}}=\infty .
$$

6.3.4 Lemma. For any set $A$ of natural numbers, let $\varphi(A)$ be the statement "there exist increasing sequences $\left\langle t_{n}\right\rangle$ and $\left\langle s_{n}\right\rangle$ of natural numbers such that $0 \notin\left\{s_{n}: n \in \mathbf{N}\right\}$, $\sum_{n \geq 0} \frac{1}{s_{n}}$ diverges and $\left\{t_{n}+s_{m}: m, n \in \mathbf{N} \wedge m \leq n\right\} \subseteq A^{\prime \prime}$. Whenever $A_{0}, A_{1} \subseteq \mathbf{N}$, if $\varphi\left(A_{0} \cup A_{1}\right)$, then $\varphi\left(A_{0}\right)$ or $\varphi\left(A_{1}\right)$.

Proof: Let $\left\langle t_{n}\right\rangle$ and $\left\langle s_{n}\right\rangle$ be increasing sequences of natural numbers such that $0 \notin\left\{s_{n}: n \in \mathbf{N}\right\}, \sum_{n \geq 0} \frac{1}{s_{n}}$ diverges and $\left\{t_{n}+s_{m}: m, n \in \mathbf{N} \wedge m \leq n\right\} \subseteq A_{0} \cup A_{1}$. Suppose available a function $\sigma: \mathbf{N} \rightarrow\{0,1\}$ and, for every $k \in \mathbf{N}$ and $i \in\{0,1\}$, a set $D_{i}(k)$ fullfing 
- $D_{\sigma(k)}(k)$ is infinite;

- $D_{\sigma(k+1)}(k+1) \subseteq D_{\sigma(k)}(k)$; and

- given any $n \in D_{\sigma(k)}(k), n \geq k$ and $t_{n}+s_{k} \in A_{\sigma(k)}$.

Then, define $B_{i}$ to be the inverse image of $\{i\}$ under $\sigma$. As $\mathbf{N}=B_{0} \cup B_{1}$, there is a $j \in\{0,1\}$ such that $\sum_{n \in B_{j}} \frac{1}{s_{n}}$ diverges, for

$$
\sum_{n \geq 0} \frac{1}{s_{n}}=\sum_{n \in B_{0}} \frac{1}{s_{n}}+\sum_{n \in B_{1}} \frac{1}{s_{n}} .
$$

Now, if $u$ is an order-preserving enumeration for $B_{j}$, the infiniteness of the parcels inside $\left\langle D_{\sigma(k)}(k)\right\rangle$ allows the definition of $\left\langle m_{k}\right\rangle$ such that $m_{0}=\min D_{\sigma\left(u_{0}\right)}\left(u_{0}\right)$ and, for any $k \geq 0$,

$$
m_{k+1}=\min D_{\sigma\left(u_{k+1}\right)}\left(u_{k+1}\right) \backslash\left\{n \in \mathbf{N}: n \leq m_{k}\right\} .
$$

Consequently, $\left\langle m_{k}\right\rangle$ is increasing, for every $k \in \mathbf{N}, m_{k} \geq k$ and $t_{m_{k}}+s_{u_{k}} \in A_{j}$. But $\left\langle D_{\sigma(k)}(k)\right\rangle$ is $\subseteq$-decreasing thus, for any $k, n \in \mathbf{N}$ such that $k \leq n$, one has $m_{n} \in$ $D_{j}\left(u_{n}\right) \subseteq D_{j}\left(u_{k}\right)$, which proves that $t_{m_{n}}+s_{u_{k}} \in A_{j}$.

Now, for the creation of the $\left\langle D_{i}(k)\right\rangle$, given any $i \in\{0,1\}$, let

$$
D_{i}(0)=\left\{n \in \mathbf{N}: t_{0}+s_{0} \in A_{i}\right\}
$$

as $\mathbf{N}=D_{0}(0) \cup D_{1}(0)$, there must be a $\sigma(0) \in\{0,1\}$ such that $D_{\sigma(0)}(0)$ is infinite. Suppose that, for a $k \geq 0$, are avaiable a list $D_{i}(0), \ldots, D_{i}(k)$ of subsets inscribed in $\mathbf{N}$ and natural numbers $\sigma(0), \ldots, \sigma(k)$ such that

- $D_{\sigma(k)}(k)$ is infinite;

- $D_{\sigma(k+1)}(k+1) \subseteq D_{\sigma(k)}(k) ;$ and

- given any $n \in D_{\sigma(k)}(k), n \geq k$ and $t_{n}+s_{k} \in A_{\sigma(k)}$.

Let

$$
D_{i}(k+1)=\left\{n \in D_{\sigma(k)}(k): n \geq k+1 \wedge t_{n}+s_{k+1} \in A_{i}\right\}
$$

Then, by the initial hypothesis, $D_{\sigma(k)}(k)=D_{0}(k+1) \cup D_{1}(k+1)$, which proves the existence of a $\sigma(k+1) \in\{0,1\}$ such that $D_{\sigma(k+1)}(k+1)$ is infinite.

6.3.5 Corolary. $\mathscr{S}_{0}$ is a closed subset of $\beta \mathbf{N}$.

6.3.6 Lemma. $\mathscr{S}_{0}=\mathrm{cl}_{\beta \mathbf{N}}\left(\mathbf{N}^{*}+\mathscr{E}\right)$

Proof: Let $p \in \mathscr{S}_{0}$ and $A \in p$; then one can find increasing sequences $\left\langle s_{n}\right\rangle$ and $\left\langle t_{n}\right\rangle$ of natural numbers satisfying $0 \notin\left\{s_{n}: n \in \mathbf{N}\right\}$,

$$
\left\{t_{n}+s_{m}: m, n \in \mathbf{N} \wedge m \leq n\right\} \subseteq A \text { and } \quad \sum_{n \geq 0} \frac{1}{s_{n}}=\infty .
$$


If

$$
B_{0}=\left\{t_{n}: n \in \mathbf{N}\right\} \quad \text { and } \quad B_{1}=\left\{s_{n}: n \in \mathbf{N}\right\},
$$

then 6.2.2 proves the existence of a $q \in \operatorname{cl}_{\beta \mathbf{N}}\left(B_{0}\right) \cap \mathbf{N}^{*}$ and a $r \in \operatorname{cl}_{\beta \mathbf{N}}\left(B_{1}\right) \cap \mathscr{E}$. Therefore, for any $m \in \mathbf{N}$,

$$
\left\{t_{n}: n \in \mathbf{N} \wedge m \leq n\right\} \subseteq A-s_{m} ;
$$

and, since $q$ does not admit finite elements,

$$
\left\{t_{n}: n \in \mathbf{N} \wedge m \leq n\right\} \in q .
$$

Hence, $A-s_{m} \in q$ from which one can derive

$$
\left\{s_{n}: n \in \mathbf{N}\right\} \subseteq\{m \in \mathbf{N}: A-m \in q\} .
$$

Therefore, $\mathrm{cl}_{\beta \mathbf{N}} A$ is a neighbourhood of $p$ that meets $\mathbf{N}^{*}+\mathscr{S}$ by the testimony of $q+r$. Consequently, $\mathscr{S}_{0} \subseteq \operatorname{cl}_{\beta \mathbf{N}}\left(\mathbf{N}^{*}+\mathscr{E}\right)$.

Conversely, if $p \in \mathbf{N}^{*}$ and $q \in \mathscr{E}$, by choosing $A \in p+q$ the set

$$
B=\{m \geq 1: A-m \in q\}
$$

is a member of $q$ and thus admits an increasing enumeration $\left\langle s_{n}\right\rangle$ whose sum of reciprocals diverges. Moreover, given any $n \in \mathbf{N}$, the set $\bigcap_{m \leq n}\left(A-s_{m}\right)$ is infinite, for it's a member of $p$; consequently, one can find an increasing sequence $\left\langle t_{n}\right\rangle$ such that, for any $n \in \mathbf{N}$, $t_{n} \in \bigcap_{m \leq n}\left(A-s_{m}\right)$. Thus, for any $n \in \mathbf{N},\left\{t_{n}+s_{m}: m \leq n\right\} \subseteq A$; which implies that $p+q \in \mathscr{S}_{0}$. As $\mathscr{S}_{0}$ is closed, $\operatorname{cl}_{\beta \mathbf{N}}\left(\mathbf{N}^{*}+\mathscr{E}\right) \subseteq \mathscr{S}_{0}$.

6.3.7 Corolary. $\mathscr{S}_{0}$ is an ideal of $\langle\beta \mathbf{N},+\rangle$ and a right ideal of $\langle\beta \mathbf{N}, \cdot\rangle$.

Proof: As $\mathscr{E}$ is a right ideal of $\langle\beta \mathbf{N},+\rangle, \mathbf{N}^{*}+\mathscr{E}$ is a right ideal of $\langle\beta \mathbf{N},+\rangle$. Since $\mathbf{N}^{*}$ is a left ideal of $\langle\beta \mathbf{N},+\rangle, \mathbf{N}^{*}+\mathscr{E}$ is an ideal of $\langle\beta \mathbf{N},+\rangle$. Thus, $\mathscr{S}_{0}=\operatorname{cl}_{\beta \mathbf{N}}\left(\mathbf{N}^{*}+\mathscr{E}\right)$ is an ideal of $\langle\beta \mathbf{N},+\rangle$.

Now, if $p \in \mathscr{S}_{0}$ and $q \in \beta \mathbf{N}$, for any $A \in p \cdot q$ there is a $k \geq 1$ that produces increasing sequences $\left\langle s_{n}\right\rangle$ and $\left\langle t_{n}\right\rangle$ of natural numbers satisfying $0 \notin\left\{s_{n}: n \in \mathbf{N}\right\}$,

$$
\left\{t_{n}+s_{m}: m, n \in \mathbf{N} \wedge m \leq n\right\} \subseteq A / k \text { and } \quad \sum_{n \geq 0} \frac{1}{s_{n}}=\infty .
$$

But $\left\langle k \cdot t_{n}\right\rangle$ and $\left\langle k \cdot s_{n}\right\rangle$ are increasing sequences, $0 \notin\left\{k \cdot s_{n}: n \in \mathbf{N}\right\}$,

$$
\left\{k \cdot t_{n}+k \cdot s_{m}: m, n \in \mathbf{N} \wedge m \leq n\right\} \subseteq A \text { and } \quad \sum_{n \geq 0} \frac{1}{k \cdot s_{n}}=\infty .
$$

As such, one can prove that $p \cdot q \in \mathscr{S}_{0}$. Hence, $\mathscr{S}_{0}$ is a right ideal of $\langle\beta \mathbf{N}, \cdot\rangle$.

Suppose that $A$ has arbitrarily long arithmetic progressions. For any $n \geq 1$, suppose also that there are chosen a non empty finite and increasing lists $a_{0}, \ldots, a_{n-1}$ and $t_{0}, \ldots, t_{n-1}$ such that, for any $m \leq n, \operatorname{AP}_{m}\left(t_{m}, a_{m}\right) \subseteq A$. Then

$$
A_{n}=\bigcup_{m<n}\left(\operatorname{AP}_{m}\left(t_{m}, a_{m}\right) \cup\left\{k \in A: k \leq \max \operatorname{AP}_{m}\left(t_{m}, a_{m}\right)\right\}\right)
$$


is a finite set. Thus one can find natural numbers $t_{n}$ and $a_{n}$ such that $a_{0}, \ldots, a_{n}$ and $t_{0}, \ldots, t_{n}$ still increasing finite lists and $\mathrm{AP}_{n}\left(t_{n}, a_{n}\right) \subseteq A$. So, there are increasing sequences of natural numbers $\left\langle t_{n}\right\rangle$ and $\left\langle a_{n}\right\rangle$ such that, for any $n \geq 1, \mathrm{AP}_{n}\left(t_{n}, a_{n}\right) \subseteq A$. Now, for any $n \geq 1$, let $s_{n}=n$. Then,

$$
\left\{t_{n}+a_{n} \cdot s_{m}: m, n \in \mathbf{N} \backslash\{0\} \wedge m \leq n\right\} \subseteq A
$$

and $\sum_{n \geq 1} s_{n}^{-1}$ diverges.

By defining the set $\mathscr{S}$ to be the set of all $p$ whose elements produce two increasing sequences $\left\langle t_{n}\right\rangle$ and $\left\langle s_{n}\right\rangle$ and a non-decreasing sequence $\left\langle a_{n}\right\rangle$ whose parcels are natural numbers such that $0 \notin\left\{s_{n}: n \in \mathbf{N}\right\}, \sum_{n \geq 0} \frac{1}{s_{n}}$ diverges,

$$
\left\{t_{n}+a_{n} \cdot s_{m}: m, n \in \mathbf{N} \wedge m \leq n\right\} \subseteq A,
$$

the inequality $\mathscr{A} \mathscr{P} \subseteq \mathscr{S}$ holds true. Moreover, it is easy to see that $\mathscr{S}_{0} \subseteq \mathscr{S}$.

6.3.8 Lemma. $\mathscr{S}$ is a closed ideal of both $\langle\beta \mathbf{N},+\rangle$ and $\langle\beta \mathbf{N}, \cdot\rangle$.

Proof: Let $p \in \mathscr{S}$ and $q \in \beta \mathbf{N}$. If $A \in p+q$, then

$$
\{n \in \mathbf{N}: A-n \in p\} \in q,
$$

which enables someone to find a $k \in \mathbf{N}$ that produces increasing sequences $\left\langle t_{n}\right\rangle$ and $\left\langle s_{n}\right\rangle$ such that $0 \notin\left\{s_{n}: n \in \mathbf{N}\right\}$ and $\sum_{n \geq 0} s_{n}^{-1}$ is divergent, and the same $k$ also produces a non-decreasing sequence $\left\langle a_{n}\right\rangle$ such that

$$
\left\{t_{n}+a_{n} \cdot s_{m}: m, n \in \mathbf{N} \wedge m \leq n\right\} \subseteq A-k .
$$

Once $\left\langle t_{n}+k\right\rangle$ is an increasing sequence of naturals and

$$
\left\{\left(t_{n}+k\right)+a_{n} \cdot s_{m}: m, n \in \mathbf{N} \wedge m \leq n\right\} \subseteq A,
$$

$p+q \in \mathscr{S}$, settling $\mathscr{S}$ as a right ideal of $\langle\beta \mathbf{N},+\rangle$.

For any $A \in p \cdot q$,

$$
\{n \in \mathbf{N}: A / n \in p\} \in q .
$$

Thus, one can find an infinite set of $k \in \mathbf{N}$ that is member of $q$ and produces increasing sequences $\left\langle t_{n}\right\rangle$ and $\left\langle s_{n}\right\rangle$ such that $0 \notin\left\{s_{n}: n \in \mathbf{N}\right\}$ and $\sum_{n \geq 0} s_{n}^{-1}$ is divergent, and a non-decreasing sequence $\left\langle a_{n}\right\rangle$ such that

$$
\left\{t_{n}+a_{n} \cdot s_{m}: m, n \in \mathbf{N} \wedge m \leq n\right\} \subseteq A / n .
$$

Evidently, $\left\langle k \cdot t_{n}\right\rangle$ and $\left\langle k \cdot s_{n}\right\rangle$ are increasing sequences of natural numbers such that $0 \notin\left\{k \cdot s_{n}: n \in \mathbf{N}\right\}$ and $\sum_{n \geq 0}\left(k \cdot s_{n}\right)^{-1}$ is divergent, and $\left\langle k \cdot a_{n}\right\rangle$ is a non decreasing sequence of natural numbers satisfying

$$
\left\{k \cdot\left(t_{n}+a_{n} \cdot s_{m}\right): m, n \in \mathbf{N} \wedge m \leq n\right\} \subseteq A .
$$

From the above inequality, one can prove that $p \cdot q \in \mathscr{S}$ and also derive that $\mathscr{S}$ is a right ideal of $\langle\beta \mathbf{N}, \cdot\rangle$. 
Now, once $\mathbf{N}$ is inscribed in the centre of $\langle\beta \mathbf{N},+\rangle$, the proof of the fact $q+p \in \mathscr{S}$ can be reduced to the proof of $q+p \in \mathscr{S}$ for some $q \in \mathbf{N}^{*}$. If $A \in q+p$, then

$$
\{n \in \mathbf{N}: A-n \in q\} \in p ;
$$

thus, one can find increasing sequences $\left\langle t_{n}\right\rangle$ and $\left\langle s_{n}\right\rangle$ such that $0 \notin\left\{s_{n}: n \in \mathbf{N}\right\}$ and $\sum_{n \geq 0} s_{n}^{-1}$ is divergent, and a non-decreasing sequence $\left\langle a_{n}\right\rangle$ such that

$$
\left\{t_{n}+a_{n} \cdot s_{m}: m, n \in \mathbf{N} \wedge m \leq n\right\} \subseteq\{k \in \mathbf{N}: A-k \in q\} .
$$

Hence, for any $n \in \mathbf{N}$, the set

$$
A_{n}=\bigcap_{m \leq n}\left(A-\left(t_{n}+a_{n} \cdot s_{m}\right)\right)
$$

is an element of $q$ and, because $q$ was assumed to be ultrafilter member of $\mathbf{N}^{*}$, it is also an infinite set. Thus, for any $n \in \mathbf{N}$ one can find a $y_{n} \in A_{n}$ such that the produced sequence $\left\langle y_{n}\right\rangle$ is increasing, since $A_{n}$ is infinite. As such, $\left\langle t_{n}+y_{n}\right\rangle$ is an increasing sequence of natural numbers and

$$
\left\{t_{n}+y_{n}+a_{n} \cdot s_{m}: m, n \in \mathbf{N} \wedge m \leq n\right\} \subseteq A .
$$

Hence, $q+p \in \mathscr{S}$ from which one settles $\mathscr{S}$ as an ideal of $\langle\beta \mathbf{N}, \cdot\rangle$.

Yet assuming $q$ as a free ultrafilter for the same reason given above, let $A \in q \cdot p$; then

$$
\{n \in \mathbf{N}: A / n \in q\} \in p ;
$$

thus, one can find increasing sequences $\left\langle t_{n}\right\rangle$ and $\left\langle s_{n}\right\rangle$ such that $0 \notin\left\{s_{n}: n \in \mathbf{N}\right\}$ and $\sum_{n \geq 0} s_{n}^{-1}$ is divergent, and a non-decreasing sequence $\left\langle a_{n}\right\rangle$ such that

$$
\left\{t_{n}+a_{n} \cdot s_{m}: m, n \in \mathbf{N} \wedge m \leq n\right\} \subseteq\{k \in \mathbf{N}: A / k \in q\} .
$$

Thus, for any $n \in \mathbf{N}, A_{n}^{\prime}=\bigcap_{m<n} A /\left(t_{n}+a_{n} \cdot s_{m}\right)$ is an element of $q$ and consequently, as $q$ was assumed to be a free ultrafilter, an infinite set. Thus, the infiniteness of $A_{n}^{\prime}$ allows, for any $n \in \mathbf{N}$, someone find an $y_{n} \in A_{n}^{\prime}$ such that the produced sequence $\left\langle y_{n}\right\rangle$ is increasing. As such, $\left\langle t_{n} \cdot y_{n}\right\rangle$ and $\left\langle y_{n} \cdot a_{n}\right\rangle$ are increasing sequence of natural numbers such that

$$
\left\{t_{n} \cdot y_{n}+y_{n} \cdot a_{n} \cdot s_{m}: m, n \in \mathbf{N} \wedge m \leq n\right\} \subseteq A .
$$

Therefore $q \cdot p \in \mathscr{S}$, settling $\mathscr{S}$ as an ideal of $\langle\beta \mathbf{N}, \cdot\rangle$.

6.3.9 Lemma. For any $k \geq 3$, suppose that, whenever disposable a set of naturals $B$ such that $\varphi(B)$ - where $\varphi$ is as stated in lemma (6.3.4) -, B contains arithmetic progressions of length $k$. Then, every set of positive naturals whose sum of reciprocals diverges contains arithmetic progressions of length $k$.

Proof: Let $\left\langle s_{n}\right\rangle$ be an order-preserving enumeration for a set of naturals $A$ whose sum of reciprocals diverges and, recursively, define $t_{0}=1$ and, for any $n \in \mathbf{N}, t_{n+1}=$ $2\left(t_{n}+s_{n+1}\right)$. The set

$$
B=\left\{t_{n}+s_{m}: m, n \in \mathbf{N} \wedge m \leq n\right\}
$$


contains, by the hypothesis, arithmetic sequences of length $k \geq 3$; thus, one can find natural numbers $a, b, m$ and $n$ such that $m \leq n$ and $a+(k-1) b=t_{n+1}+s_{m+1}$. If $a \leq t_{n+1}$ occurs, then $a \leq t_{n}+s_{n}$. Setting

$$
l=\max \left\{j<k-1: a+j b \leq t_{n}+s_{n}\right\},
$$

one has

$$
b=a+(l+1) b-(a+l b) \geq t_{n+1}-\left(t_{n}+s_{n}\right)>t_{n+1}-\left(t_{n}+s_{n+1}\right)=t_{n}+s_{n+1} .
$$

Thus,

$$
t_{n+1}+s_{m+1}<t_{n+1}+s_{n+1}<t_{n+1}+t_{n}+s_{n+1}<a+2 b \leq a+(k-1) b,
$$

which is absurd.

6.3.10 Theorem. [Hin88] The following are equivalent:

(ET1) for each natural number $k$ and for every $A \subseteq \mathbf{N} \backslash\{0\}$ such that $\sum_{n \in A} n^{-1}$ diverges, there are natural numbers $a$ and $b$ such that $\operatorname{AP}_{k}(a, b) \subseteq A$;

ET2 $\mathscr{E} \subseteq \mathscr{A} \mathscr{P}$

ET3 $\mathscr{S}_{0} \subseteq \mathscr{A} \mathscr{P}$; and

ET4 $\mathscr{S} \subseteq \mathscr{A} \mathscr{P}$.

Proof: The sequence of the proof will be $(\mathrm{ET} 1) \Rightarrow \mathrm{ET} 2, \mathrm{ET} 2 \Rightarrow \mathrm{ET} 3, \mathrm{ET} 3 \Rightarrow$ $(\mathrm{ET} 1),(\mathrm{ET} 1) \Rightarrow \mathrm{ET} 4$ and ET4 $\Rightarrow \mathrm{ET} 3$.

The fact that (ET1) implies ET2 can be derived from corollaries 6.2.2 and 6.3.4.

$(\mathrm{ET} 2 \Rightarrow \mathrm{ET} 3)$ Now, if $\mathscr{E}$ is a subset of $\mathscr{A} \mathscr{P}$, then the fact that $\mathscr{A} \mathscr{P}$ is a left ideal of $\langle\beta \mathbf{N},+\rangle$ testifies that

$$
\mathbf{N}^{*}+\mathscr{E} \subseteq \mathbf{N}^{*}+\mathscr{A} \mathscr{P} \subseteq \mathscr{A} \mathscr{P}
$$

thus, as $\mathscr{A} \mathscr{P}$ is a closed subset of $\beta \mathbf{N}$,

$$
\mathscr{S}_{0}=\operatorname{cl}_{\beta \mathbf{N}}\left(\mathbf{N}^{*}+\mathscr{S}\right) \subseteq \mathscr{A} \mathscr{P}
$$

which settles ET3.

$(\mathrm{ET} 3 \Rightarrow(\mathrm{ET} 1))$ Now, for any natural number $k$, let $P(k)$ is the statement "whenever $A \subseteq \mathbf{N}$ and $\varphi(A)$ - where $\varphi$ is as in lemma (6.3.4) - , A contains a arithmetic progression of length $k^{\prime \prime}$. Being $A$ a set of positive natural numbers such that $\varphi(A)$, the lemma (6.3.4) and the corolary 6.2.2 testify the existence of an ultrafilter $p \in \operatorname{cl}_{\beta \mathbf{N}}(A) \cap \mathscr{S}_{0}$ that, when assuming ET3, must be an element of $\mathscr{A} \mathscr{P}$. Thus, ET3 implies $\forall k(k \in \mathbf{N} \rightarrow P(k))$.

Assuming ET3, for any natural number $k \geq 3$, if $A$ is a set of positive natural numbers whose sum of reciprocals diverges, then the lemma 6.3.9 implies the existence of an arithmetical progressions of length $k$ inside $A$; otherwise, as $\forall k(k \in \mathbf{N} \rightarrow P(k))$ 
can be derived from ET3, one can pick any $k_{0} \geq 3$ and prove that $A$ has arithmetical progressions of length $k_{0}$, which also prove that $A$ has arithmetical progressions of length $k .^{2}$

$((\mathrm{ET} 1) \Rightarrow \mathrm{ET} 4)$ Assuming (ET1), once one can select an ultrafilter $p$ inside $\mathscr{S}$, every $A \in p$ will testify the existence an increasing sequence of positive natural numbers $\left\langle s_{n}\right\rangle$, an increasing sequence $\left\langle t_{n}\right\rangle$ composed by natural numbers and a non-decreasing sequence of natural numbers $\left\langle a_{n}\right\rangle$ such that $\sum_{n \geq 0} \frac{1}{s_{n}}$ diverges and

$$
\left\{t_{n}+a_{n} \cdot s_{m}: m, n \in \mathbf{N} \wedge m \leq n\right\} \subseteq A .
$$

By the hypothesis, for any $k \geq 0$ there are $a, b \in \mathbf{N}$ fulfilling $\operatorname{AP}_{k}(a, b) \subseteq\left\{s_{n}: n \in \mathbf{N}\right\}$. Thus, there is a $n \in \mathbf{N}$ satisfying $a+(k-1) b=s_{n}$; moreover, any natural $j<k$ produces some $m \leq n$ in such way that $s_{m}=a+j b$. Once

$$
t_{n}+a_{n} \cdot s_{m}=t_{n}+a_{n}(a+j b)=\left(t_{n}+a \cdot a_{n}\right)+j\left(b \cdot a_{n}\right),
$$

$\operatorname{AP}_{k}\left(t_{n}+a \cdot a_{n}, b \cdot a_{n}\right) \subseteq A$. Thus, $p \in \mathscr{A} \mathscr{P}$ which proves that $\mathscr{S} \subseteq \mathscr{A} \mathscr{P} .^{3}$

(ET4 $\Rightarrow$ ET3) Lastly, assuming ET4 and selecting any set $A$ inside some ultrafilter $p \in \mathscr{S}_{0}$, one can find increasing sequences of natural numbers $\left\langle s_{n}\right\rangle$ and $\left\langle t_{n}\right\rangle$ such that $0 \notin\left\{s_{n}: n \in \mathbf{N}\right\}, \sum_{n \geq 0} \frac{1}{s_{n}}$ diverges and

$$
\left\{t_{n}+s_{m}: m, n \in \mathbf{N} \wedge m \leq n\right\} \subseteq A .
$$

If $\left\langle a_{n}\right\rangle$ is the constant sequence whose parcels are all equal to 1 , one has

$$
t_{n}+a_{n} \cdot s_{m}=t_{n}+a_{n}(a+j b)=\left(t_{n}+a \cdot a_{n}\right)+j\left(b \cdot a_{n}\right),
$$

which proves that $p \in \mathscr{A} \mathscr{P}$.

\subsection{Final Remarks}

6.4.1 Lemma. The set $\mathbf{N}^{*} \backslash \mathscr{D}$ is a left ideal of $\langle\beta \mathbf{N},+\rangle$ and $\langle\beta \mathbf{N}, \cdot\rangle$.

Proof: For every $p \in \mathbf{N}^{*} \backslash \mathscr{D}$ and $q \in \beta \mathbf{N}$, let $B$ be a set of $p$ whose natural density is naught. Let $f: \mathbf{N} \rightarrow \mathbf{I}$ given by

$$
f(n)=\frac{|B \cap[1, n]|}{n} .
$$

\footnotetext{
${ }^{2}$ Here it was proved that ET3 implies $\forall k(k \in \mathbf{N} \rightarrow P(k))$ and $\forall k(k \in \mathbf{N} \rightarrow P(k))$ implies $\forall k(k \in$ $\mathbf{N} \rightarrow S R(k)$ ), where $S R(k)$ is the statement 'whenever $A \subseteq \mathbf{N}$ is a set of positive natural whose series of reciprocals diverges, then $A$ must contains arithmetical progressions of length $k$ '. It's weaker than to prove that ET3 implies $\forall k(k \in \mathbf{N} \rightarrow(P(k) \rightarrow S R(k)))$.

${ }^{3}$ The reader should be aware that, as it was already proved that $\mathscr{A} \mathscr{P} \subseteq \mathscr{S}$, hence ET4 could be replaced equivalently by the assertion $\mathscr{S}=\mathscr{A} \mathscr{P}$.
} 
Evidently, $\lim f(n)=0$; thus, for any $m \in \mathbf{N}$ is possible to find a $n_{m} \in \mathbf{N}$ such that $f(n)<m^{-2}$, whenever $n>n_{m}$. Let $B_{m}=\left\{n \in B: n>n_{m}\right\}$. As $B \backslash B_{m}$ is finite, for every $m \in \mathbf{N}, B_{m} \in p$. Let

$$
S=\bigcup_{m \in \mathbf{N}}\left(m+B_{m}\right) \quad \text { and } \quad P=\bigcup_{m \in \mathbf{N}} m \cdot B_{m}
$$

For each $m \in \mathbf{N},(m+B)-m \in p$. Thus,

$$
\{m \in \mathbf{N}: S-m \in p\} \in q,
$$

which proves that $S \in q+p$.

Analogously, one can prove that $P \in q \cdot p^{4}$.

Given $m \in \mathbf{N}$ and $n \in B_{m}$, let $r \in \mathbf{N}$ satisfying $m+n<r$. As $n>n_{m}$, one can prove that $n_{m}<r$ and $f(r)<m^{-2}$. Therefore, for a fixed $r \geq 1$, the number of $m \in \mathbf{N}$ satisfying $m+n<r$, with $n \in B_{m}$, is at most $\sqrt{f(r)^{-1}}$. Once $n \in B \cap[1, r]$, the number of possible $n$ satisfying $m+n<r$ must be, at most, $f(r) \cdot r$. Thus, the number of possibles $m$ and $n$ such that $n \in B_{m}$ and $m+n<r$ must be limited by $r \sqrt{f(r)}$. Consequently,

$$
\frac{|S \cap[1, r]|}{r} \leq \sqrt{f(r)}
$$

Thus, $d(S)=0$. Analogously, one can prove that $d(P)=0$. Thus, $q+p$ and $q \cdot p$ are elements of $\mathbf{N}^{*} \backslash \mathscr{D}$, which settles the desired.

As was already observed, $\mathbf{N}$ is inscribed into the centre of both $\langle\beta \mathbf{N},+\rangle$ and $\langle\beta \mathbf{N}, \cdot\rangle$; this fact combined with lemma 6.4.1 proves that:

6.4.2 Corolary. [vD91] The centre of $\langle\beta \mathbf{N},+\rangle$ and $\langle\beta \mathbf{N}, \cdot\rangle$ is $\mathbf{N}$.

\subsubsection{Szemerédi's Theorem revised}

6.4.3 Lemma. The following statements are equivalent:

(6.4.3.1). If $A$ is a set of natural numbers having a positive natural upper density, then $A$ contains arbitrarily long arithmetical sequences;

(6.4.3.2). If $A$ is a set of natural numbers having a positive Banach upper density, then $A$ contains arbitrarily long arithmetical sequences;

Proof: As $d \leq \mathcal{b}$, the implication $(6.4 .3 .2) \Rightarrow(6.4 .3 .1)$ is trivial. Reciprocally, if $\mathscr{D}$ is a subset of $\mathscr{A} \mathscr{P}$, then

$$
\mathscr{B}=\operatorname{cl}_{\beta \mathbf{N}}\left(\mathbf{N}^{*}+\mathscr{D}\right) \subseteq \operatorname{cl}_{\beta \mathbf{N}}\left(\mathbf{N}^{*}+\mathscr{A} \mathscr{P}\right) \subseteq \operatorname{cl}_{\beta \mathbf{N}} \mathscr{A} \mathscr{P}=\mathscr{A} \mathscr{P},
$$

\footnotetext{
${ }^{4}$ Actually, in a discrete semigroup $S$, given $p, q \in \beta S$, a set $A$ is in $q \cdot p$ if and only if one can find a $B \in q$ and a family $\left\langle C_{s}\right\rangle_{s \in B}$ inside $p$ such that $\bigcup_{s \in B} s C_{s} \subseteq A$.
} 
which concludes the proof.

With the help of the Riesz-Markov-Kakutani Theorem, for any subset $A$ of $\mathbf{N}$ whose Banach density is positive, [Zir12] constructed a Radon measure $\mu$ on $\beta \mathbf{N}$ such that its support is contained in $\mathscr{A} \mathscr{P}$ and

$$
\mu\left(\operatorname{cl}_{\beta \mathbf{N}} A\right)=\mathfrak{b}(A) .
$$

Within this framework, the Szemerédi's theorem is equivalent to the equality $\mu(\mathscr{A} \mathscr{P})=1$.

Suppose that $\varphi$ is a predicate concerning subsets of $\mathbf{N}$ that satisfy (i), (ii) and (iii) of the corollary (6.2.2) and $\mu$ is a Radon measure of $\beta \mathbf{N}$ such that for any $A \subseteq \mathbf{N}$

(a) $\varphi(A)$; and

(b) $\mu\left(\mathrm{cl}_{\beta \mathbf{N}} A\right)>0$.

are equivalent. Then, the same arguments given by Zirnstein can be used to prove that $\varphi(A)$ implies that $A$ contains arbitrarily long arithmetical progressions if and only if $\mu(\mathscr{A} \mathscr{P})=1$.

6.4.4 Open Problem. Give a proof of the Szemerédi's theorem in the fashion proposed by Zirnstein.

\subsubsection{Some open questions}

Remember that the prime number theorem states that

$$
\lim _{n \rightarrow \infty} \frac{\pi(x) \log (x)}{x}=1,
$$

where $\pi(x)$ is the quantity of primes less than or equal to $x$.

As a consequence of the prime number theorem, $d(\mathbf{P})=0$, where $\mathbf{P}$ is the collection of all prime numbers; but in [GT08] Ben Green and Terence Tao proved that $\mathbf{P}$ contains arbitrarily long arithmetic progressions. In the proof, they considered the function $t$ : $\wp(\mathbf{N}) \rightarrow \mathbf{I}$ given by

$$
t(A)=\limsup \frac{|A \cap[1, n]|}{\pi(n)},
$$

The function $t$ is a upper density of $\mathbf{N}$ and what was actually proved is that whenever a set of natural $A$ numbers has a positive value under $t$, then $A$ contains arbitrarily long arithmetical sequences. So far, then, the following question seems to arise easily.

6.4.5 Open Problem. How intimate is the relation of upper densities and the ideal $\mathscr{A} \mathscr{P}$ ? 
Apparently not so close as one could desire. Following the work done in [Pv91], for any set $A \subseteq \mathbf{N}$, let $\mathcal{A}(A)$ be the collection of all $B \subseteq \mathbf{N}$ containing $A$ as a subset for which there are non empty list of natural numbers $a_{0}, \ldots, a_{k-1}$ and $b_{0}, \ldots, b_{k-1}$ such that

$$
B=\bigcup_{i<k} a_{i}+b_{i} \cdot \mathbf{N}
$$

Given any $A \subseteq \mathbf{N}, \mathcal{A}(A)$ is a sublattice of $\langle\wp(\mathbf{N}), \subseteq\rangle$ such

(BL1) $\mathbf{N} \in \mathcal{A}(A) ;$ and

(BL2) for any natural numbers $a$ and $b$ with $a \geq 1$ are natural numbers, $B$ is an element of $\mathcal{A}(A)$ if and only if $a \cdot B+b$ is an element of $\mathcal{A}(A)$.

Thus, for any natural numbers $a \geq 1$ and $b, \mathcal{A}(a \cdot A+b)=\mathcal{A}(A)$. The Buck upper density is the function $b: \wp(\mathbf{N}) \rightarrow \mathbf{I}$ given by

$$
b(A)=\inf _{B \in \mathcal{A}(A)} \bar{d}(B) .
$$

From (BL1) and (BL2), $\boldsymbol{b}$ is an upper density function of $\mathbf{N}$. Let $a \geq 1$ and $b$ be natural numbers and $A=a \mathbf{N}+b$. Define $S=\{n+n !: n \in \mathbf{N}\}$ and, for each $k \in \mathbf{N}, n_{k}=a k+b$. Then, as for any $k \in \mathbf{N}, n_{k}+n_{k} ! \in A \cap S$, the set $A \cap S$ is infinite. Thus, $\mathcal{b}(A)=1$. But $S$ does not contain a arithmetic progression of length 3 ; indeed, if $1 \leq a_{0}<a_{1}<a_{2}$ is an arithmetic progression inside $S$, then one could, for any $j \in\{0,1,2\}$, find $n_{k} \in \mathbf{N}$ such that $n_{j}+n_{j}$ ! $=a_{j}$. As $b=n_{1}+n_{1}$ ! $-n_{0}-n_{0}$ ! is the rate of this progression, one easily see that

$$
\begin{aligned}
a_{2} & =a_{0}+2 b=\left(n_{0}+n_{0} !\right)+2\left[\left(n_{1}+n_{1} !\right)-\left(n_{0}+n_{0} !\right)\right]=2\left(n_{1}+n_{1} !\right)-\left(n_{0}+n_{0} !\right)< \\
& <2\left(n_{1}+n_{1} !\right)<\left(n_{1}+1\right)+\left(n_{1}+1\right) ! \leq n_{2}+n_{2} !=a_{2},
\end{aligned}
$$

which is absurd. Thus, $A$ is a set of positive Buck density that does not possess arithmetical sequences of length 3 .

A the following reformulation of (6.4.5) seems valid and is open.

6.4.6 Open Problem. For which class of upper densities of $\mathbf{N}$ the analogous of the Szemerédi's Theorem is true? I.e., what are the properties that an upper density $\delta$ of $\mathbf{N}$ must have to ensure that, for each $A \subseteq \mathbf{N}$, if $\delta(A)>0$, then $A$ contains arbitrarily long arithmetic progressions (or equivalently, $\left.\mathscr{D}_{\delta} \subseteq \mathscr{A} \mathscr{P}\right)$ ?

6.4.7 Open Problem. What algebraic-topological properties can ensure that an closed ideal $I$ of $\langle\beta \mathbf{N},+\rangle$ is of the form $\mathscr{D}_{\delta}$, for some upper density $\delta$ of $\mathbf{N}$ ?

6.4.8 Open Problem. Does every closed ideal of $\langle\beta \mathbf{N},+\rangle$ circumscribed by $\mathscr{A} \mathscr{P}$ is the support of some regular Borel measure of $\beta \mathbf{N}$ ?

Recently, the combinatorial and number theoretical communities has given attention to sets of the form

$$
\mathscr{R}=\{\mathfrak{p} \in \beta \mathbf{N}: \forall A(A \in \mathfrak{p} \rightarrow \varphi(A))\}
$$


where $\varphi$ is a nice combinatorial or number theoretical property discoursing about subsets of $\mathbf{N}$. This format of set has been central to the theory developed in [Bag12; Bag15; Bag14; dNB16] to deal with Diophantine equations of the format $p\left(x_{0}, \ldots, x_{k}\right)=0$ for a $p \in \mathbf{Z}\left[X_{0}, \ldots, X_{k}\right] ; \varphi$ in the mentioned works is in the form

$$
\exists x_{0} \exists x_{1} \ldots \exists x_{k}\left(x_{0} \in A \wedge \ldots \wedge x_{k} \in A \rightarrow p\left(x_{0}, \ldots, x_{k}\right)=0\right) .
$$

A property $\varphi$ about subsets of $A$ is called a partition regular property if, for any $B \subseteq A$ and any partition $B_{0}, \ldots, B_{k-1}$ of $B, \varphi(B)$ implies the existence of some $i<k$ such that $\varphi\left(B_{i}\right)$. As proved by the van der Waerden theorem, to contain arbitrarily long arithmetical progression is a partition regular property. The Szemerédi theorem implies that having positive Banach (or natural) density is partition regular.

Partition regularity of non linear equations are far from understood. As an example, the following simple-to-enunciate problem is still open:

6.4.9 Open Problem. (see [Ber96, Question 11], [HLS03, Question 3] and [Guy04, Sec. E29]) Given a partition of $\mathbf{N}$ into finitely many colours, does exist $x, y \in \mathbf{N}$ such that $\{x, y, x+y, x y\}$ is monochromatic? 


\section{Bibliography}

[Aus60] Joseph Auslander. On the proximal relation in topological dynamics. Proceedings of the American Mathematical Society, 11(6):890-895, 1960.

[Bag12] Lorenzo Luperi Baglini. Hyperintegers and nonstandard techniques in combinatorics of numbers. PhD thesis, Università Degli Studi di Siena, 2012.

[Bag14] Lorenzo Luperi Baglini. Partition regularity of nonlinear polynomials: a nonstandard approach. Integers, pages A-30, 2014.

[Bag15] Lorenzo Luperi Baglini. A nonstandard techinique in combinatorial number theory. European J. Combin., pages 71-80, 2015.

[Ber96] V. Bergelson. Ergodic Ramsey Theory, page 1-62. London Mathematical Society Lecture Note Series. Cambridge University Press, 1996.

[dNB16] Mauro di Nasso and Lorenzo Luperi Baglini. Ramsey properties of nonlinear diophantine equations. ArXiv e-prints, 2016.

[Ell58] Robert Ellis. Distal transformation groups. Pacific Journal of Mathematics, 8(3):401-405, 1958.

[Ell60] Robert Ellis. A semigroup associated with a transformation group. Transactions of the American Mathematical Society, 94(2):272-281, 1960.

[Eng64] Ryszard Engelking. Remarks on real-compact spaces. Fund. Math., 55:287304, 1964.

[Eng89] Ryszard Engelking. General Topology. Heldermann Verlag Berlin, 1989.

[ET36] Paul Erdôs and Paul Turán. On some sequences of integers. London Mathematical Society, 11:261-264, 1936.

[FKO82] Hillel Furstenberg, Yitzhak Katznelson, and Donald Samuel Ornstein. The ergodic theoretical proof of szemerédi's theorem. Bull. Amer. Math. Soc., 7:527-552, 1982.

[Fre80] Gottlob Frege. The Foundations of Arithmetic. Northwestern University Press, 2th edition, 1980. 
[Fri64] Orrin Frink. Compactifications and semi-normal spaces. American Journal of Mathematics, 83(3):602-607, 1964.

[Fur77] Hillel Furstenberg. Ergodic behavior of diagonal measures and a theorem of szemerédi on arithmetic progressions. Journal d'Analyse Mathématique, 31:204-256, 1977.

[Fur81] Hillel Furstenberg. Recurrence in ergodic theory and combinatorial number theory. Princeton University Press, 1st edition, 1981.

[Ger77] J. L. Gerver. The sum of reciprocals of a set of integers with no arithmetic progressions of $k$ terms. Proc. Amer. Math. Soc., 62:211-214, 1977.

[Ger83] J. L. Gerver. Irregular sets of integers generated by the greedy algorithm. Math. Comp., 40:667-676, 1983.

[GJ60] Leonard Gilmar and Meyer Jerison. Rings of continuous functions. Princeton, N.J., Van Nostrand, 1st edition, 1960.

[GK39] Israïl Moiseevich Gelfand and Andrei Nikolaevich Kolmogorov. On ring of continuous functions on topological spaces. C.R. Acad. Sci. URSS, 22, 1939.

[GT08] Ben Green and Terence Tao. The primes contain arbitrarily long arithmetic progressions. Annals of Mathematics, 167:481-547, 2008.

[Guy04] Richard Guy. Unsolved Problems in Number Theory. Springer, 3 edition, 2004.

[Hal78] Paul Richard Halmos. Measure Theory. Graduate Texts in Mathematics. Spring Verlag, 1978.

[Hin88] Neil Hindman. Some equivalents of the Erdôs sum of reciprocals conjecture. Europ. J. Combinatorics, 9:39-47, 1988.

[HLS03] Neil Hindman, Imre Leader, and Dona Strauss. Open problems in partition regularity. Combinatorics, Probability and Computing, page 571-583, 2003.

[HLvM14] Klaas Pieter Hart, Leon Luo, and Jan van Mill. Unions of F-spaces. Topology Proc., 43:293-300, 2014.

[HS12] Neil Hindman and Donna Strauss. Algebra in Stone-Čech compactification. De Gruyter, 2 edition, 2012.

[Juh80] István Juhász. Cardinal functions in topology, ten years later. Mathematical Centre tracts. Mathematisch Centrum, 1980.

[Kun11] Kenneth Kunen. Set Theory. College Publications, 2011.

[LAS95] J. Arthur Seebach Jr. Lynn Arthur Steen. Counterexamples in Topology. Dover Books on Mathematics. Dover Publications, 1995.

[Lev02] Azriel Levy. Basic Set Theory. Dover, 1 edition, 2002. 
[LT15] P. Leonetti and S. Tringali. On the notions of upper and lower density. ArXiv e-prints, 2015.

[MAM07] Carlos Gustavo Moreira, Alexander Arbieto, and Carlos Matheus. Aspectos ergódicos da teoria dos números. Publicações Matemáticas. IMPA, 1st edition, 2007.

[Nac47] Leopoldo Nachbin. Une propriété caractéristique des algebres booléennes. Portugaliae Mathematica, 6:115-118, 1947.

[Num52] Katsui Numakura. On bicompact semigroups. Math. J. Okayama University, 1:99-108, 1952.

[Par63] I. I. Parovičenko. A universal bicompactum of weight §. Dokl. Akad. Nauk SSSR, 150:36-39, 1963.

[Pv91] Milan Paštéka and Tibor Šalát. Buck's measure density and sets of positive integers containing arithmetic progression. Mathematica Slovaca, 41:283-293, 1991.

[PW88] Jack R. Porter and R. Grant Woods. Extension and Absolutes of Hausdorff Spaces. Springer-Verlag, 1988.

[Rus08] Bertrand Russell. Mysticism and Logic and Other Essays. Project Gutenberg, 2008.

[Sap29] E. Sapir. The status of linguistics as a science. Language, 5(4):207-214, 1929.

[Sha97] Stewart Shapiro. Philosophy of Mathematics: Structure and Ontology. Oxford University Press, 1997.

[Sho67] Joseph Shoenfield. Mathematical Logic. Addison-Wesley series in logic. Addison-Wesley Publishing Company, 1 edition, 1967.

[Son78] Dan Sonnenschein. A general theory of asymptotic densities. Master's thesis, Simon Fraser University, 1978.

[Sto35] Marshall Harvey Stone. Applications of the theory of Boolean rings to general topology. Trans. Am. Math. Soc., 2, 1935.

[Sun89] Shu-Hao Sun. Another Appoach to Extensions of Continuous Mappings. Bull. Austral. Math. Soc., 39:1-9, 1989.

[Sze75] Endre Szemerédi. On sets of integers containing no k elements in arithmetic progression. Acta Arithmetica, 22:199-245, 1975.

[Taı̌52] Asan Dabsovich Taı̆manov. On extension of continuous mappings of topological spaces. Matematicheskii Sbornik, 73:287-304, 1952.

[Ten15] Gérald Tenenbaum. Introduction to analytic and probabilistic number theory. Graduate Studies in Mathematics. Amer. Math. Soc., 2015. 
[Tyc30] Andrey Tychonoff. Über die topologische erweiterung von räumen. Mathematische Annalen, 102(1):544-561, 1930.

[Ul'77] V.M. Ul'janov. Solution of a basic problem on compactifications of Wallman type. Soviet Math. Dokl., 18:567-571, 1977.

[Ury25] Pavel Urysohn. Über die Mächtigkeit der zusammenhängenden Mengen. Mathematische Annalen, 94:262-295, 1925.

[Č37] Eduard Čech. On bicompact spaces. Annals of Mathematics, 38, 1937.

[Č59] Eduard Čech. Topologické prostory. Praha, 1959.

[vD91] Eric van Douwen. The Čech-stone compactification of a discrete cancellative groupoid. Topology and its Applications, 39, 1991.

[vDvM78] Eric van Douwen and Jan van Mill. Parovičenko's characterization of $\beta \mathbf{N}-\mathbf{N}$ implies ch. Proc. Amer. Math. Soc., 72:539-541, 1978.

[vdW27] Bartel Leendert van der Waerden. Beweis einer baudetschen vermutung. Nieuw Archief voor Wiskunde, 19:212-216, 1927.

[vM84] Jan van Mill. An introduction to $\beta \omega$. In Kenneth Kunen and Jerry Vaughan, editors, Handbook of set-theoretic topology. Elsevier, 1984.

[Vul52] Boris Zakharovich Vulih. On the extension of continuous functions on topological spaces. Matematicheskii Sbornik, 72:167-170, 1952.

[Wal38] Henry Wallman. Lattices and topological spaces. Annals of Mathematics, 39(1), 1938.

[Wal74] Russel C. Walker. The Stone-Čech Compactification. Springer Berlin Heidelberg, 1974.

[Wit58] Ludwig Wittengenstein. Philosophical Investigations. Basil Blackwell, 2 edition, 1958.

[Zaı̌67] V. I. Zaĭcev. On the theory of tychonoff spaces. Vestnik Moskov. Univ. Ser. I Mat. Meh, 3:48-57, 1967.

[Zir12] Heinrich-Gregor Zirnstein. Formulating szemerédi's theorem in terms of ultrafilters. ArXiv e-prints, 2012. 


\section{Index}

Axiom of choice, 8

Base, 18

normal base of closed sets, 21 normal base of open sets, 21 of closed sets, 18

of open sets, 18

Binary relation, 5

Boolean algebra, 13

Centre of a semigroup, 48

Clousure, 17

compact space, 29

Compactification, 38

extention of a, 38

complementation, 12

Completely separated sets, 21

Dense subset, 17

Density

Banach upper density, 63

Buck upper density, 79

natural upper density, 65

upper density function, 61

Density of a topological space, 43

Equivalence, 4

$\mathrm{F}_{\sigma}$ set, 17

Filter, 10

generated by a set, 11

maximal, 11

prime, 11

proper, 10

Function, 5 bijection, 5

injective, 5

surjective, 5

Functionally closed set, 20

Functionally open set, 20

Følner sequence, 64

$\mathrm{G}_{\delta}$ set, 17

Hasse diagram, 14

Hausdorff space, 19

Homeomorphism, 19

Ideal, 10, 50

generated by a set, 11

proper, 10

Ideal: left, 50

Ideal:right, 50

Identity map, 26

Implication, 4

Inclusion map, 26

Indexation, 5

Infimum, 7

Interior, 17

Intersection, 4

Kuratowski-Zorn's Lemma, 8

Lattice, 8

bounded, 8

complete, 14

distributive, 10

normal, 31

Limit

P-limit, 24 
of a sequence, 24

of a filter, 25

Majorant, 7

Maximal element, 7

Maximum, 7

Minimal element, 7

Minimal ideal of a semigroup, 50

Minimum, 7

Minorant, 7

Neighbourhood, 17

Normal space, 22

Ordered pair, 5

Partial order, 7

Piecewise syndetic set, 56

Power set, 5

Progression, 6 arithmetic, 6

Projection map, 27
Quantifier, 4

existential, 4

universal, 4

quasi-compact, 29

Regular space, 20

Reminder of a compactification, 43

Stone's duality, 30

Subbase, 18

Supremum, 7

Syndetic set, 56

$\mathrm{T}_{1}$ space, 19

Transversal set, 8

Tychonoff space, 21

Ultrafilter, 11

Union, 4

Weight of a topological space, 38

Zero-dimensional space, 32 